\title{
Premixed flames modelled with thermally sensitive intermediate branching kinetics
}

\author{
J. W. DOLD* \\ School of Mathematics, University of Manchester, Manchester M60 1QD, UK
}

(Received 28 October 2006; in final form 15 February 2007)

\begin{abstract}
The foundations of a relatively simple two-step kinetic scheme for flame chemistry are outlined, involving a model chain branching process that should adopt the activation temperature of a ratelimiting branching reaction in order to offer a broad approximation for hydrocarbon flames. A model energetic intermediate reactant then acts as a buffer between fuel consumption and the release of heat, as the intermediate is converted into products through a completion reaction step. By taking the rate of the latter reaction to be linear in the concentration of the intermediate, which is consistent with the final state being an equilibrium in a broader chemical system, a form of the model is arrived at which admits asymptotic solutions in a thermodiffusive context with constant coefficients. These are developed to second order for large values of the activation energy of the branching reaction and are found to involve the same trends that are seen for lean methane and hydrogen flames calculated using detailed chemical and transport models. Linear stability analysis identifies the ranges of Lewis numbers in which cellular or oscillatory instability can arise, with the latter form of instability disappearing above a threshold heat of reaction. These and the underlying flame solutions themselves depend on the heat of reaction and the degree of heat loss but not on the activation temperature of the branching reaction, to leading order. Near the limit of flammability a direct parallel arises with one-step kinetic models for premixed flames.
\end{abstract}

Keywords: Premixed flames; Two-step kinetic model; Intermediate chain-branching chemistry; Flame stability

\section{Introduction}

\subsection{One-step and chain-branching models}

Much of the progress that has been made in developing the theory of combustion, so far, has been based on a one-step model for the chemistry and the use of asymptotic methods for approximating solutions, see for example [1-11]. By assuming that a single global reaction, such as

$$
\mathrm{F}+\mathrm{M} \rightarrow \mathrm{P}+\mathrm{M} \quad: \quad k_{1}=A_{1} \mathrm{e}^{-T_{\mathrm{A} 1} / T}
$$

satisfies an Arrhenius rate law with rate constant $k_{1}$, in which the activation temperature $T_{\mathrm{A} 1}$ is taken to be large, an asymptotic structure emerges. All of the chemistry is found to occur in a thin sheet at a thermally sensitive rate. In the case of the reaction (1) the fuel species $F$ is converted into the product species $\mathrm{P}$ at the reaction sheet, after collision with a molecule $\mathrm{M}$ which represents any molecule that is needed to trigger the reaction but that is not changed by

*E-mail: John.Dold@manchester.ac.uk

Combustion Theory and Modelling

ISSN: 1364-7830 (print), 1741-3559 (online) @ 2007 Taylor \& Francis

http://www.tandf.co.uk/journals

DOI: $10.1080 / 13647830701294599$ 


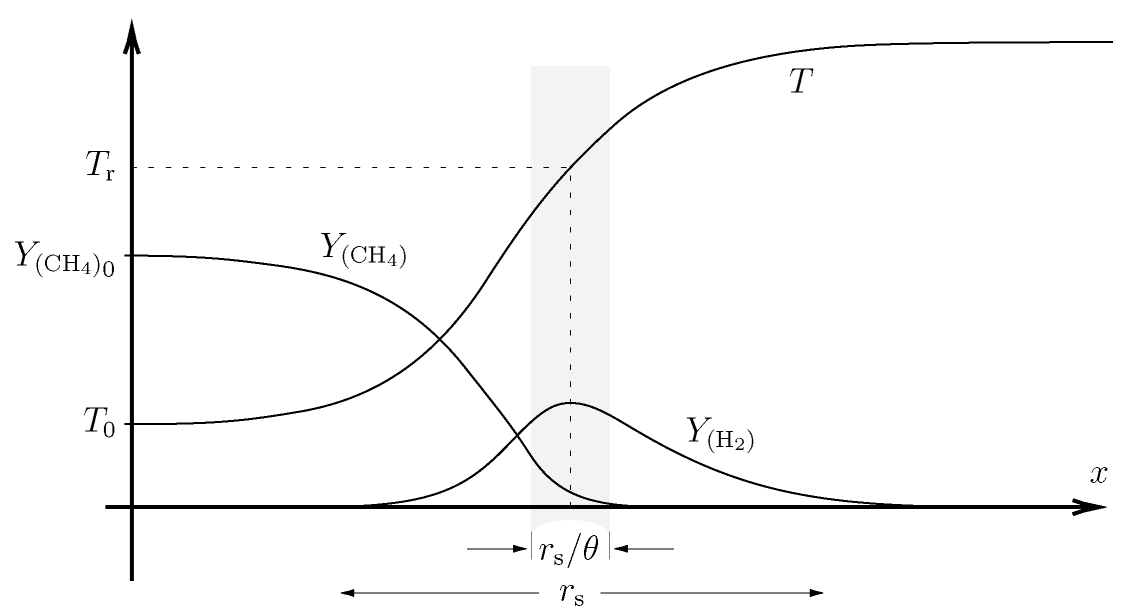

Figure 1. A schematic illustration of the structure of a lean adiabatic planar premixed methane-air flame (following for example [15]) showing a reference length-scale $r_{\mathrm{s}}$ for diffusion and consumption of intermediate reactants and a region of branching reaction (shaded) of approximate length $r_{\mathrm{s}} / \theta$ where methane is converted into hydrogen and other energetic intermediate species (as well as some reaction products) around a reference temperature $T_{\mathrm{r}}$.

the reaction. The model is then simple enough to admit explicit matched asymptotic solutions in many cases, offering transparent descriptions for the structure, stability and behaviour of many forms of flame. Perhaps because the one step model has been relatively successful alternative chemical kinetic models have not been given similar levels of attention from the point of view of asymptotic analysis $[9,11]$.

The one step model cannot capture all of the properties of real flames, although it certainly does capture two major features, namely exothermic chemistry and one form of nonlinear dependence of the reaction rate on temperature. A feature of hydrogen and hydrocarbon oxidation that is completely absent is the presence of chemical radicals (molecules with unbonded electrons) and other intermediate species that act in concert to catalyse the production of each other at the expense of the initial fuel and oxidant [11]. Such intermediate reactants can also act as a buffer between fuel consumption and heat production. Studies of lean premixed hydrocarbon flames using reduced-chemistry approaches (for example [12-15]) suggest that fuel tends to be used up almost completely at some intermediate point within the flame structure, as illustrated in figure 1. At this stage it has been converted almost entirely into products and other energetic species which diffuse and ultimately recombine to form equilibrium reaction products and heat.

In a one-step model the fuel is converted directly into products and heat, and the possible structural and dynamical contribution of intermediate species is therefore lost; if the activation temperature is large, the maximum temperature appears at (or very near) the point at which the fuel is consumed. This is generally not the case for real hydrocarbon flames where the recombination of intermediate species continues to release heat so that a maximum temperature can be generated after the stage at which the deficient reactant of the initial mixture is consumed.

The following simple generalization of the one-step model

$$
\begin{array}{lll}
\mathrm{F}+\mathrm{Z} \rightarrow 2 \mathrm{Z} & : & k_{\mathrm{B}}=A_{\mathrm{B}} \mathrm{e}^{-T_{\mathrm{B}} / T} \\
\mathrm{Z}+\mathrm{M} \rightarrow \mathrm{P}+\mathrm{M} & : & k_{\mathrm{C}}=A_{\mathrm{C}}
\end{array}
$$

in which $\mathrm{Z}$ is a generic representation of a 'pool' of energetic intermediate species, is similar to a model first proposed by Zel'dovich [16]. These intermediates might loosely be referred to as 'radicals' although they would generally consist of many different species, only a small proportion of which may in fact be active radicals. Also, as a simple model, we can treat $\mathrm{Z}$ as 
if it is a single intermediate species, taking part in only two elementary reactions which have the overall effect of converting the fuel $\mathrm{F}$ into the product $\mathrm{P}$. In its broadest sense $\mathrm{Z}$ can be thought of as representing the deviation of a 'pool' of reactants from their equilibrium state (as will be discussed later).

The first 'branching' reaction in (2) represents an autocatalytic attack by the intermediate $\mathrm{Z}$ on the fuel $\mathrm{F}$. We may take this reaction to occur in a thermally sensitive way with activation temperature $T_{\mathrm{B}}$ in the formula for the rate constant $k_{\mathrm{B}}$ in (2). The intermediate is converted into products by the final reaction which we may assume has a rate constant $k_{\mathrm{C}}$ that does not change with temperature. The effect of these two reactions is that, at a high enough temperature (above some 'crossover' value [9]), there can be a chain-branching growth in the concentration of intermediate species. Below the crossover temperature the intermediate is removed from the system (converted into products by the second reaction) too quickly for a chain-branching process to occur.

The model orginally used by Zel'dovich $[3,16]$ to describe planar premixed flames can be characterized as

$$
\begin{array}{lll}
\mathrm{F}+\mathrm{Z} \rightarrow 2 \mathrm{Z} & : & k_{\mathrm{B}}=A_{\mathrm{B}} \mathrm{e}^{-T_{\mathrm{B}} / T} \\
\mathrm{Z}+\mathrm{Z} \rightarrow 2 \mathrm{P} & : & k_{\mathrm{C}}=A_{\mathrm{C}}
\end{array}
$$

in which the rate of the recombination reaction varies nonlinearly (in fact quadratically) with the concentration of $\mathrm{Z}$. Perhaps the most basic difference between this model and (2) is that branching of the intermediate species becomes possible at all low enough positive concentrations of Z, for any temperature. Thus the model (3) suffers from a form of the 'cold-boundary difficulty' [1], as does the one-step model (1), while the model (2) does not.

\subsection{Chain-branching kinetics}

Before discussing the branching models (2) and (3) in more detail, it is useful briefly to review some pertinent aspects of hydrogen and hydrocarbon chemistry. Readers familiar with the kinetics of hydrocarbon oxidation may wish to skip this section.

Chain branching is a well-known feature of hydrocarbon oxidation, determining the progress of spatially homogeneous self-ignition (amongst other things). The most important branching and propagation reactions that affect chain branching in hydrogen oxidation are considered $[9,17]$ to be

$$
\begin{array}{rrr}
\mathrm{H}^{\cdot}+\mathrm{O}_{2} & \rightarrow \mathrm{OH}^{\cdot}+\mathrm{O}^{:} & -0.727 \mathrm{ev} \\
\mathrm{O}^{\ddagger}+\mathrm{H}_{2} & \rightarrow \mathrm{OH}^{\cdot}+\mathrm{H}^{\cdot} & -0.081 \mathrm{ev} \\
\mathrm{OH}^{\cdot}+\mathrm{H}_{2} \rightarrow \mathrm{H}_{2} \mathrm{O}+\mathrm{H}^{\cdot} & 0.651 \mathrm{ev}
\end{array}
$$

in which the dots represent unbonded electrons. These elementary reaction steps serve to

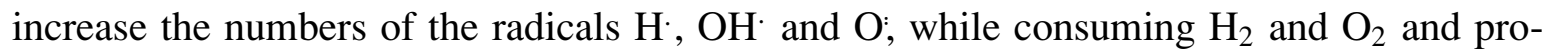
ducing $\mathrm{H}_{2} \mathrm{O}$. Many other reactions are involved in both hydrogen and hydrocarbon oxidation; an illustrative sample of the full set of elementary reactions that participate in the oxidation of methane is presented in table 1, along with approximate rate data for each reaction step selected from a variety of sources using the NIST database [18].

The numbers listed on the right of each reaction step, both in (4) and in table 1, give the energy released by the chemical change in electron volts per molecule ${ }^{1}$ at the standard

\footnotetext{
${ }^{1}$ One electron volt is about $1.602177 \times 10^{-19}$ joules so that one electron volt per molecule represents $1 \mathrm{ev} \times A_{0} \approx$ $96485.3 \mathrm{~J} \mathrm{~mole}^{-1}$, where Avogadro's number is $A_{0} \approx 6.022137 \times 10^{23}$ molecules per mole.
} 
Table 1. A representative selection of elementary reactions in the oxidation of methane with rate constants $k=A\left(\frac{T}{1000 \mathrm{~K}}\right)^{\chi} \mathrm{e}^{-T_{\mathrm{A}} / T}$. Activation temperatures $T_{\mathrm{A}}$ are measured in degrees Kelvin while the column headed ev gives the activation energies measured in electron volts per molecule. Units of the pre-exponential factors $A$ are appropriate for species concentrations measured in moles per $\mathrm{cm}^{3}$ and reaction progress rates in terms of moles of chemical steps per $\mathrm{cm}^{3}$ per second. For a density of $M \approx 8.018 \times 10^{-6}$ moles per $\mathrm{cm}^{3}$ (as appropriate for an ideal gas) and a reaction of order $n$, the column headed $M^{n} k_{1500}$ provides a relative measure of the rate constant of each reaction-step at a temperature of $1500 \mathrm{~K}$ at atmospheric pressure. The column headed $\left[h_{i \mathrm{o}} W_{i}\right]_{-}^{+}$lists the energy released, in electron volts, through one molecular step at $298.15 \mathrm{~K}$.

\begin{tabular}{|c|c|c|c|c|c|c|}
\hline Reaction & $M^{n} k_{1500}$ & $A$ & $\chi$ & $T_{\mathrm{A}}$ & $\mathrm{ev}_{\mathrm{A}}$ & {$\left[h_{i \mathrm{o}} W_{i}\right]_{-}^{+}$} \\
\hline $\mathrm{CH}_{4}+\mathrm{M} \rightarrow \mathrm{CH}_{3}+\mathrm{H}^{\cdot}+\mathrm{M}$ & $1.98 \times 10^{-6}$ & $1.18 \times 10^{23}$ & -8.2 & 59200 & 5.101 & -4.545 \\
\hline $\mathrm{CH}_{4}+\mathrm{O}_{2} \rightarrow \mathrm{CH}_{3}+\mathrm{HO}_{2}$ & $1.34 \times 10^{-5}$ & $3.97 \times 10^{13}$ & 0 & 28600 & 2.465 & -2.308 \\
\hline $\mathrm{CH}_{4}+\mathrm{O}^{\prime} \rightarrow \mathrm{OH}^{-}+\mathrm{CH}_{3}$ & 342 & $3.39 \times 10^{14}$ & 0 & 6230 & 0.537 & -0.108 \\
\hline $\mathrm{CH}_{4}+\mathrm{H} \rightarrow \mathrm{H}_{2}+\mathrm{CH}_{3}$ & 167 & $2.64 \times 10^{13}$ & 2.59 & 5057 & 0.436 & -0.027 \\
\hline $\mathrm{CH}_{4}+\mathrm{OH} \rightarrow \mathrm{H}_{2} \mathrm{O}+\mathrm{CH}_{3}$ & 497 & $3.57 \times 10^{12}$ & 3.21 & 793 & 0.068 & 0.625 \\
\hline $\mathrm{CH}_{4}+\mathrm{HO}_{2} \rightarrow \mathrm{H}_{2} \mathrm{O}_{2}+\mathrm{CH}_{3}$ & 0.187 & $1.13 \times 10^{13}$ & 0 & 12400 & 1.069 & -0.854 \\
\hline $\mathrm{CH}_{3}+\mathrm{H}^{\cdot} \rightarrow \mathrm{CH}_{2}+\mathrm{H}_{2}$ & 24.4 & $6.03 \times 10^{13}$ & 0 & 7600 & 0.655 & -0.235 \\
\hline $\mathrm{CH}_{3}+\mathrm{H}_{2} \rightarrow \mathrm{CH}_{4}+\mathrm{H}$ & 7.95 & $8.04 \times 10^{11}$ & 2.7 & 4451 & 0.384 & 0.027 \\
\hline $\mathrm{CH}_{3}+\mathrm{O}^{:} \rightarrow \mathrm{CH}_{2} \mathrm{O}+\mathrm{H}^{-}$ & 5420 & $8.43 \times 10^{13}$ & 0 & 0 & 0 & 3.034 \\
\hline $\mathrm{CH}_{3}+\mathrm{OH}^{-} \rightarrow \mathrm{CH}_{2}+\mathrm{H}_{2} \mathrm{O}$ & 94.7 & $1.11 \times 10^{12}$ & 3 & 1400 & 0.121 & 0.416 \\
\hline $\mathrm{CH}_{3}+\mathrm{OH} \rightarrow \mathrm{CH}_{2} \mathrm{O}+\mathrm{H}_{2}$ & 0.113 & $8.21 \times 10^{10}$ & -0.53 & 5440 & 0.469 & 3.115 \\
\hline $\mathrm{CH}_{2}+\mathrm{O}_{2} \rightarrow \mathrm{CO}_{2}+2 \mathrm{H}$ & 2.67 & $4.14 \times 10^{11}$ & -3.3 & 1440 & 0.124 & 3.564 \\
\hline $\mathrm{CH}_{2}+\mathrm{OH}^{-} \rightarrow \mathrm{CH}_{2} \mathrm{O}+\mathrm{H}^{\cdot}$ & 1160 & $1.81 \times 10^{13}$ & 0 & 0 & 0 & 3.351 \\
\hline $\mathrm{CH}_{2}+\mathrm{H} \rightarrow \mathrm{CH}+\mathrm{H}_{2}$ & 213 & $6.03 \times 10^{12}$ & 0 & 900 & 0.078 & 0.106 \\
\hline $\mathrm{CH}^{\vdots}+\mathrm{H}_{2} \rightarrow \mathrm{CH}_{2}+\mathrm{H}^{\cdot}$ & 4000 & $1.87 \times 10^{14}$ & 0 & 1650 & 0.142 & -0.106 \\
\hline $\mathrm{CH}+\mathrm{O}_{2} \rightarrow \mathrm{CHO}+\mathrm{O}^{:}$ & 643 & $1.00 \times 10^{13}$ & 0 & 0 & 0 & 3.124 \\
\hline $\mathrm{CH}_{2} \mathrm{O}+\mathrm{H} \rightarrow \mathrm{CHO}+\mathrm{H}_{2}$ & 550 & $9.17 \times 10^{12}$ & 1.62 & 1090 & 0.094 & 0.607 \\
\hline $\mathrm{CH}_{2} \mathrm{O}+\mathrm{OH}^{-} \rightarrow \mathrm{CHO}+\mathrm{H}_{2} \mathrm{O}$ & 1430 & $1.19 \times 10^{13}$ & 1.18 & -225 & -0.019 & 1.258 \\
\hline $\mathrm{CHO}+\mathrm{H}^{-} \rightarrow \mathrm{CO}^{\prime}+\mathrm{H}_{2}$ & 5810 & $9.04 \times 10^{13}$ & 0 & 0 & 0 & 3.856 \\
\hline $\mathrm{CHO}+\mathrm{OH}^{-} \rightarrow \mathrm{CO}+\mathrm{H}_{2} \mathrm{O}$ & 6560 & $1.02 \times 10^{14}$ & 0 & 0 & 0 & 4.507 \\
\hline $\mathrm{CHO}+\mathrm{O}_{2} \rightarrow \mathrm{CO}^{*}+\mathrm{HO}_{2}$ & 60.3 & $2.14 \times 10^{11}$ & 2.38 & -768 & -0.066 & 1.575 \\
\hline $\mathrm{CHO}+\mathrm{M} \rightarrow \mathrm{CO}+\mathrm{H}+\mathrm{M}$ & 51.1 & $1.57 \times 10^{14}$ & 0 & 7930 & 0.683 & -0.663 \\
\hline $\mathrm{CO}+\mathrm{OH}^{-} \rightarrow \mathrm{CO}_{2}+\mathrm{H}^{-}$ & 23.3 & $1.48 \times 10^{11}$ & 1.55 & -402 & -0.035 & 1.078 \\
\hline $\mathrm{CO}_{2}+\mathrm{H}^{-} \rightarrow \mathrm{CO}^{\prime}+\mathrm{OH}^{-}$ & 1.37 & $1.51 \times 10^{14}$ & 0 & 13300 & 1.146 & -1.078 \\
\hline $\mathrm{O}_{2}+\mathrm{M} \rightarrow 2 \mathrm{O}+\mathrm{M}$ & $4.92 \times 10^{-13}$ & $1.81 \times 10^{15}$ & -1 & 59400 & 5.119 & -5.165 \\
\hline $\mathrm{H}_{2}+\mathrm{M} \rightarrow 2 \mathrm{H}+\mathrm{M}$ & $6.62 \times 10^{-11}$ & $2.88 \times 10^{15}$ & -1.4 & 52500 & 4.524 & -4.519 \\
\hline $\mathrm{O}_{2}+\mathrm{H}^{\cdot} \rightarrow \mathrm{OH}+\mathrm{O}^{-}$ & 45.5 & $1.99 \times 10^{14}$ & 0 & 8460 & 0.729 & -0.727 \\
\hline $\mathrm{OH}+\mathrm{O}^{\prime} \rightarrow \mathrm{O}_{2}+\mathrm{H}$ & 737 & $1.45 \times 10^{13}$ & 0 & 353 & 0.030 & 0.727 \\
\hline $\mathrm{H}_{2}+\mathrm{O}^{\circ} \rightarrow \mathrm{OH}^{-}+\mathrm{H}^{-}$ & 121 & $5.25 \times 10^{12}$ & 2.67 & 3160 & 0.272 & -0.081 \\
\hline $\mathrm{OH}^{-}+\mathrm{H}^{\cdot} \rightarrow \mathrm{H}_{2}+\mathrm{O}^{:}$ & 67.1 & $1.23 \times 10^{12}$ & 2.8 & 1950 & 0.168 & 0.081 \\
\hline $\mathrm{H}_{2}+\mathrm{OH}^{\cdot} \rightarrow \mathrm{H}_{2} \mathrm{O}+\mathrm{H}^{\cdot}$ & 263 & $6.46 \times 10^{12}$ & 1.6 & 1660 & 0.143 & 0.651 \\
\hline $\mathrm{H}_{2} \mathrm{O}+\mathrm{H}^{-} \rightarrow \mathrm{H}_{2}+\mathrm{OH}$ & 5.38 & $2.85 \times 10^{13}$ & 1.6 & 9720 & 0.838 & -0.651 \\
\hline $2 \mathrm{OH} \rightarrow \mathrm{H}_{2} \mathrm{O}+\mathrm{O}^{:}$ & 391 & $3.96 \times 10^{12}$ & 1.14 & 50 & 0.004 & 0.732 \\
\hline $\mathrm{H}_{2} \mathrm{O}+\mathrm{O}^{:} \rightarrow 2 \mathrm{OH}$ & 12.4 & $3.95 \times 10^{13}$ & 1.14 & 8680 & 0.748 & -0.732 \\
\hline $\mathrm{HO}_{2}+\mathrm{H} \rightarrow \mathrm{H}_{2}+\mathrm{O}_{2}$ & 1710 & $4.28 \times 10^{13}$ & 0 & 710 & 0.061 & 2.281 \\
\hline $\mathrm{HO}_{2}+\mathrm{H}^{-} \rightarrow 2 \mathrm{OH}$ & 8100 & $1.69 \times 10^{14}$ & 0 & 440 & 0.038 & 1.473 \\
\hline $\mathrm{HO}_{2}+\mathrm{H} \rightarrow \mathrm{H}_{2} \mathrm{O}+\mathrm{O}$ & 1090 & $3.01 \times 10^{13}$ & 0 & 866 & 0.075 & 2.205 \\
\hline $\mathrm{HO}_{2}+\mathrm{OH} \rightarrow \mathrm{H}_{2} \mathrm{O}+\mathrm{O}_{2}$ & 2190 & $2.89 \times 10^{13}$ & 0 & -250 & -0.022 & 2.932 \\
\hline $\mathrm{H}_{2} \mathrm{O}_{2}+\mathrm{M} \rightarrow 2 \mathrm{OH}+\mathrm{M}$ & 1.82 & $1.21 \times 10^{17}$ & 0 & 22900 & 1.973 & -2.219 \\
\hline $\mathrm{H}+\mathrm{O}_{2}+\mathrm{M} \rightarrow \mathrm{HO}_{2}+\mathrm{M}$ & 0.651 & $1.99 \times 10^{15}$ & -1.12 & 0 & 0 & 2.238 \\
\hline $\mathrm{H}+\mathrm{OH}^{-}+\mathrm{M} \rightarrow \mathrm{H}_{2} \mathrm{O}+\mathrm{M}$ & 32.3 & $1.41 \times 10^{17}$ & -2 & 0 & 0 & 5.170 \\
\hline
\end{tabular}

temperature of $298.15 \mathrm{~K}$. The electron volt is a natural unit for measuring the energy of an electronic bond. At the higher temperatures that are typical of flames, differences in the heat capacities per molecule of the species on the left and on the right sides of each step lead to a change in the amount of energy that is released, but this change is normally only a small fraction of an electron volt. For example, using data on heat capacities [19], the changes in energy of each of the reaction steps in (4) is found to be less than $\frac{1}{10}$ ev at typical flame temperatures of about $1500 \mathrm{~K}$ to $2500 \mathrm{~K}$. 
Thus, at all reasonable reaction temperatures, the first (branching) reaction in (4) is mildly endothermic while the second (also branching) is practically neutral and the third (chain propagation) reaction is mildly exothermic. It is worth comparing the energies of these reaction steps with the energy that would be released in each of the overall chemical changes

$$
\begin{array}{rlrl}
\mathrm{O}_{2} & \rightarrow 2 \mathrm{O} & & -5.165 \mathrm{ev} \\
\mathrm{H}_{2} & \rightarrow 2 \mathrm{H} \cdot & -4.519 \mathrm{ev} \\
\mathrm{H}_{2}+\mathrm{O}_{2} & \rightarrow 2 \mathrm{OH} & -0.808 \mathrm{ev} \\
\mathrm{H}_{2}+\mathrm{O}_{2} \rightarrow \mathrm{H}_{2} \mathrm{O}+\mathrm{O}^{:} & -0.076 \mathrm{ev} \\
2 \mathrm{H}_{2}+\mathrm{O}_{2} \rightarrow \mathrm{OH}^{\cdot}+\mathrm{H}^{\cdot}+\mathrm{H}_{2} \mathrm{O} & -0.157 \mathrm{ev} \\
2 \mathrm{H}_{2}+\mathrm{O}_{2} \rightarrow 2 \mathrm{H}_{2} \mathrm{O} & 5.012 \mathrm{ev}
\end{array}
$$

which are not necessarily elementary reaction steps.

It can be seen that complete dissociation of oxygen $\mathrm{O}_{2}$ and hydrogen $\mathrm{H}_{2}$ into atoms absorbs a lot of energy; direct dissociation of $\mathrm{H}_{2}$ or $\mathrm{O}_{2}$ is relatively rare, having a very large activation temperature (as seen in table 1). The combination of $\mathrm{H}_{2}$ and $\mathrm{O}_{2}$ into hydroxyl radicals $\mathrm{OH}$. requires relatively little energy; while rearranging them into water $\mathrm{H}_{2} \mathrm{O}$ and an oxygen atom $\mathrm{O}$ : is practically thermally neutral, because the energy generated by producing $\mathrm{H}_{2} \mathrm{O}$ almost completely balances the energy required to produce the dissociated $\mathrm{O}$ : In fact, the production of the energetic hydrogen atom $\mathrm{H}$. through the third elementary reaction in (4) is partly facilitated by the energy that is simultaneously released in producing the water molecule $\mathrm{H}_{2} \mathrm{O}$.

The fifth chemical change listed in (5) represents one possible cycle of chain branching, arrived at simply by adding together the first three reactions in (4). It causes very little change in energy, especially when compared with the energy released in the final complete oxidation process which involves exactly the same initial combination of hydrogen and oxygen molecules.

Elementary reactions that contribute to breaking down hydrocarbons into radicals and other intermediate species in the oxidation of hydrocarbons include the following kinds of additional steps [17]

$$
\begin{array}{rlr}
\mathrm{RH}+\mathrm{O} & \rightarrow \mathrm{R} \cdot+\mathrm{OH}^{\cdot} & -0.108 \mathrm{ev} \\
\mathrm{RH}+\mathrm{H} & \rightarrow \mathrm{R}+\mathrm{H}_{2} & -0.027 \mathrm{ev} \\
\mathrm{RH}+\mathrm{OH}^{\cdot} \rightarrow \mathrm{R}+\mathrm{H}_{2} \mathrm{O} & 0.625 \mathrm{ev} \\
\mathrm{RH}+\mathrm{HO}_{2} \rightarrow \mathrm{R}+\mathrm{H}_{2} \mathrm{O}_{2} & -0.854 \mathrm{ev} \\
\mathrm{H}_{2} \mathrm{O}_{2}+\mathrm{M} \rightarrow 2 \mathrm{OH}+\mathrm{M} & -2.219 \mathrm{ev} \\
\mathrm{H}+\mathrm{O}_{2}+\mathrm{M} \rightarrow \mathrm{HO}_{2}+\mathrm{M} & 2.238 \mathrm{ev}
\end{array}
$$

where $\mathrm{RH}$ is any alkane, $\mathrm{R} \cdot$ is an alkyl radical, $\mathrm{HO}_{2}$ is the hydroperoxyl radical and $\mathrm{H}_{2} \mathrm{O}_{2}$ is hydrogen peroxide. The change in energy for the first four steps is based on $\mathrm{R}$ being a methyl radical $\mathrm{CH}_{3}$, although roughly the same pattern of values would arise for most alkanes. The last three steps are considered to contribute to chain-branching in hydrocarbon oxidation at relatively low temperatures between about $900 \mathrm{~K}$ and about $1100 \mathrm{~K}$ [17]. At higher temperatures the first three steps are more important in contributing to the initial attack on the alkane, with the first two steps generating hydrogen and hydroxyl which link into the hydrogen branching process in (4). As can be seen in table 1, there are numerous other steps and sequences of steps in which radicals attack the fuel $\mathrm{RH}$ and break it down into radicals based on carbon and hydrogen as well as other energetic intermediates, including hydrogen $\mathrm{H}_{2}$ itself. The energy needed to produce such intermediates is often balanced by energy released in generating a product with a lower energy of formation, such as $\mathrm{H}_{2} \mathrm{O}, \mathrm{CO}$ or $\mathrm{CO}_{2}$.

To illustrate some aspects of the changes in energy that are possible during hydrocarbon oxidation, we can consider the following overall conversions in methane oxidation, chosen 
fairly randomly

$$
\begin{array}{lr}
\mathrm{CH}_{4}+2 \mathrm{O}_{2} \rightarrow \mathrm{CO}+2 \mathrm{OH}+\mathrm{H}_{2}+\mathrm{O}_{2} & -0.439 \mathrm{ev} \\
\mathrm{CH}_{4}+2 \mathrm{O}_{2} \rightarrow \mathrm{CO}+\mathrm{OH}^{\cdot}+\mathrm{H}^{\cdot}+\mathrm{H}_{2} \mathrm{O} & 0.212 \mathrm{ev} \\
\mathrm{CH}_{4}+2 \mathrm{O}_{2} \rightarrow \mathrm{CO}_{2}+\mathrm{OH}^{\cdot}+\mathrm{H}^{\cdot}+\mathrm{H}_{2}+\frac{1}{2} \mathrm{O}_{2} & 0.639 \mathrm{ev} \\
\mathrm{CH}_{4}+2 \mathrm{O}_{2} \rightarrow \mathrm{CO}_{2}+2 \mathrm{H}_{2} \mathrm{O} & 8.315 \mathrm{ev}
\end{array}
$$

In each of the first three of these changes the energy that is absorbed or released in producing an intermediate mixture is less than $8 \%$ of the energy that is released in the final complete oxidation process, for exactly the same initial combinations of molecules. Some other intermediate and complete chemical changes in the oxidation of ethane, propane, ethanol and acetylene are

$$
\begin{array}{rlr}
\mathrm{C}_{2} \mathrm{H}_{6}+\frac{7}{2} \mathrm{O}_{2} & \rightarrow 2 \mathrm{CO}+3 \mathrm{OH}+\frac{3}{2} \mathrm{H}_{2}+\mathrm{O}_{2} & 0.210 \mathrm{ev} \\
\mathrm{C}_{2} \mathrm{H}_{6}+\frac{7}{2} \mathrm{O}_{2} & \rightarrow 2 \mathrm{CO}_{2}+3 \mathrm{H}_{2} \mathrm{O} & 14.807 \mathrm{ev} \\
\mathrm{C}_{3} \mathrm{H}_{8}+5 \mathrm{O}_{2} & \rightarrow 3 \mathrm{CO}+2 \mathrm{OH}+3 \mathrm{H}_{2}+\frac{5}{2} \mathrm{O}_{2} & 1.543 \mathrm{ev} \\
\mathrm{C}_{3} \mathrm{H}_{8}+5 \mathrm{O}_{2} & \rightarrow 3 \mathrm{CO}_{2}+4 \mathrm{H}_{2} \mathrm{O} & 21.175 \mathrm{ev} \\
\mathrm{C}_{2} \mathrm{H}_{6} \mathrm{O}+3 \mathrm{O}_{2} & \rightarrow 2 \mathrm{CO}+3 \mathrm{OH}+\frac{3}{2} \mathrm{H}_{2}+\mathrm{O}_{2} & -1.360 \mathrm{ev} \\
\mathrm{C}_{2} \mathrm{H}_{6} \mathrm{O}+3 \mathrm{O}_{2} & \rightarrow 2 \mathrm{CO}_{2}+3 \mathrm{H}_{2} \mathrm{O} & 13.236 \mathrm{ev} \\
\mathrm{C}_{2} \mathrm{H}_{2}+\frac{5}{2} \mathrm{O}_{2} & \rightarrow 2 \mathrm{CO}+2 \mathrm{OH}+\mathrm{H}_{2}+\mathrm{O}_{2} & -0.438 \mathrm{ev} \\
\mathrm{C}_{2} \mathrm{H}_{2}+\frac{5}{2} \mathrm{O}_{2} & \rightarrow 2 \mathrm{CO}_{2}+\mathrm{H}_{2} \mathrm{O} & 8.315 \mathrm{ev}
\end{array}
$$

Rearrangements of the products on the right sides of the first of each of these pairs of chemical changes, along the lines of (7), would produce similar patterns in which a variety of possible changes all involve relatively small changes in energy. This does not prove that chain branching in hydrocarbon oxidation necessarily involves relatively little change in energy, but it does show that it could proceed in that way.

Returning to table 1, four significant features are worth noting. Firstly, the rates of exothermic reaction steps, those with a positive value of $\left[h_{i_{0}} W_{i}\right]_{-}^{+}$, do not vary strongly with temperature. In most cases the activation temperature is either small or zero (sometimes even assigned a relatively small negative value). These reactions include all 'completion' steps, in which intermediates are converted into final products, as well as 'recombination' steps, in which intermediates recombine into fuel or oxidant. On the other hand, strong thermal sensitivity in the rate of an elementary reaction step tends to correlate with endothermicity (or negative values of $\left[h_{i_{0}} W_{i}\right]^{+}$) often having an activation energy that is similar in magnitude to $\left[h_{i_{0}} W_{i}\right]^{+}$.

Secondly, the two key branching steps in the oxidation of hydrogen and the propagation step in (4) have activation temperatures of $8460 \mathrm{~K}, 3160 \mathrm{~K}$ and $1660 \mathrm{~K}$. The latter two also have a relatively weak power-law dependence on temperature, with powers of 2.67 and 1.6, respectively. The overall rate of the branching cycle should be limited by the rate of the slowest participating reaction, namely the first, with the largest activation temperature. The overall branching process in hydrogen oxidation, at high enough temperatures [17], should therefore have an effective activation temperature of about $8460 \mathrm{~K}$.

Thirdly, the numerous reaction steps through which methane is broken down into radicals and other energetic intermediate species generally have relatively low activation temperatures and they mostly involve reaction with either $\mathrm{OH}, \mathrm{H} \cdot$ or $\mathrm{O}$. Once again, the key controlling step in the chain-branching production of these radicals should be the relatively slow reaction $\mathrm{H}^{\cdot}+\mathrm{O}_{2} \rightarrow \mathrm{OH}^{\cdot}+\mathrm{O}$ : Broadly speaking, this branching step seems to play a key role in hydrocarbon oxidation in flames, suggesting that overall branching processes for both hydrogen and hydrocarbon oxidation, at high enough temperatures, are likely to be thermally sensitive with an effective activation temperature of approximately $8460 \mathrm{~K}$. 
It should also be mentioned that many reactions serve to remove radicals, usually as exothermic completion steps with rates that do not vary much with temperature. If the rate of production of radicals and other intermediates falls below their rate of removal, as would happen if the temperature is lowered sufficiently, then chain-branching must stop.

The fourth point to note is that methane chemistry, as illustrated in table 1, involves more steps that consume $\mathrm{OH}^{*}, \mathrm{H}^{-}$and $\mathrm{O}^{*}$ than hydrogen chemistry, suggesting that hydrocarbon oxidation should be more efficient at removing radicals than hydrogen oxidation. Moreover the branching process in (4) requires hydrogen atoms $\mathrm{H}$ and molecules $\mathrm{H}_{2}$ which themselves need to be produced through the breakdown of the hydrocarbon. As a result branching processes should require a higher temperature to proceed in hydrocarbon oxidation than in hydrogen oxidation.

Comparison of the second order asymptotic results of this article with numerical solutions for lean adiabatic premixed flames, as is done in the Appendix, offers the rough estimation that branching for hydrogen in air needs temperatures in excess of about $750 \mathrm{~K}$ while branching for methane in air requires above about $1100 \mathrm{~K}$. These estimates must involve higher order asymptotic errors, in addition to simplifications in modelling density and transport processes, that may well underpredict the minimum required temperature for branching, but because analogous inaccuracies apply to both the hydrogen and the methane flames the temperature difference of roughly $350 \mathrm{~K}$ is probably a good estimate.

Hydrogen and hydrocarbon oxidation involve many further aspects that are not mentioned in this short outline and that cannot be captured by simplified models of the form of (2) or (3), although it is worth noting that a study by Seshadri et al [14] offers a reduced hydrogen scheme that is broadly similar to these models; it has one branching step and one completion step with significantly more complicated rate formulae that lead to a more complex asymptotic description for flame structure. Westbrook and Dryer [20] discuss many more processes that feature in hydrocarbon oxidation.

\subsection{Further background and role of this article}

Symbolically, a more general form of the branching and recombination kinetic scheme (2) can be written as

$$
\begin{array}{lll}
\mathrm{F}+\mathrm{Z} \rightarrow\left(1-\mu_{\mathrm{F}}\right) \mathrm{F}+\left(1+\mu_{\mathrm{Z}}\right) \mathrm{Z} & : & k_{\mathrm{B}}=A_{\mathrm{B}} \mathrm{e}^{-T_{\mathrm{B}} / T} \\
\mathrm{Z}+\mathrm{M} \rightarrow \mathrm{P}+\mathrm{M} & : & k_{\mathrm{C}}=A_{\mathrm{C}}
\end{array}
$$

which serves to represent an overall chain branching process in which some positive fraction $\mu_{\mathrm{F}}$ of the fuel molecules that are involved in the process is converted into another positive fraction $\mu_{Z}$ of the initially participating intermediate molecules. Since the overall chain branching process in hydrocarbons involves several steps, only a few of which actually increase the number of intermediate species, this overall, partially branching representation would appear to be more realistic than the direct conversion process represented in the schemes (2) or (3). In fact the scheme (2) and the first step of the scheme (3) are reproduced when $\mu_{\mathrm{F}}=\mu_{\mathrm{Z}}=1$.

In this article we examine the more general scheme (9), allowing for varying degrees of chain branching. As will be seen a simple scaling unites all degrees of chain branching into a single dimensionless version of the model conservation equations (provided $\mu_{\mathrm{F}}>0$ and $\mu_{\mathrm{Z}}>0$ ) so that the overall asymptotic structure of premixed flames is the same as it would be for the simpler model (2), scaled suitably. The rate constants $A_{\mathrm{B}}, A_{\mathrm{C}}$ and $T_{\mathrm{B}}$ in the model are left arbitrary, but it seems likely that reasonable values can be chosen to model hydrocarbon oxidation with intermediate chemistry in a relatively simple way; a suitable value for $T_{\mathrm{B}}$ is likely to be about $8460 \mathrm{~K}$. Questions concerning relevant quantitative values for thermal, 
kinetic and transport parameters in practical applications are left open for further study, although the Appendix offers some rough estimates. Instead, we examine the generic nature of planar premixed flames driven by kinetics of the form (2) or (9) using constant density and constant transport coefficients as well as linear heat loss. This is clearly a significant development from the modelling of flames using one-step kinetics although yet more generalization is undoubtedly possible.

The chain branching chemical models (2) and (9) are inherently more simple than the model (3) that was orginally used by Zel'dovich [3,16] (and subsequently by a few other authors [21-27]) to describe planar premixed flames. As well as not suffering from the cold boundary difficulty, the most important difference from a mathematical point of view is that the rate of the second completion reaction in (2) and (9) depends linearly on the mass fraction of $\mathrm{Z}$, making explicit general solutions possible whereas they would not be possible for the model scheme (3). In spite of this the model (2) has attracted relatively little attention [28-30]. In turn, the model (3) has received very little attention when compared with the one-step model.

Kapila examined the role of a model of the type (3) in spatially homogeneous selfignition [21]. Joulin et al. [22] used the mechanism to model planar premixed flames in a limiting case where the conversion of intermediates to products is fast compared with the time-scale of heat conduction, a range that was developed further by Chao and Law [23]. Mikolaitis [24] examined the model numerically under more general conditions but did not examine the limit of large activation temperature of the intermediate reaction. Tam $[25,26]$ used an asymptotic approach which reinforced earlier results of Seshadri and Peters [27] concerning stretch resistance of laminar flames driven by kinetics of the form (3). Stability of flames was not examined.

Much of the work in these articles is predicated on insisting that the dimensional concentration of the intermediate $\mathrm{Z}$ should be small in any realistic results (see for example the arguments in [23] and [25]). The approach seems to be based on the fact that the observed concentration of any one radical in real flames is normally small, along with an assumption that $\mathrm{Z}$ exclusively models radical species. It should be noted, however, that there are numerous radical species in hydrocarbon flames as well as other intermediate chemicals that are not technically radicals. These generally include significant quantities of hydrogen $\mathrm{H}_{2}$ and carbon monoxide $\mathrm{CO}$, which are also produced in the chain branching process and which react further to produce the final products.

It can also be noted that there are very many completion reactions in hydrocarbon chemistry, as seen in table 1, which work in concert to produce equilibrium reaction products. These products include significant proportions of carbon dioxide $\mathrm{CO}_{2}$, water $\mathrm{H}_{2} \mathrm{O}$ and, in lean premixed flames, oxygen $\mathrm{O}_{2}$; in rich flames, carbon monoxide $\mathrm{CO}$ and hydrogen $\mathrm{H}_{2}$. Bearing in mind the variety of intermediate reactants and the wide variety of pathways through which they can produce these final products, there is no reason to suppose that a 'lumped' intermediate reactant represented by $\mathrm{Z}$ should be converted into products at a rate that is essentially quadratic, as suggested by the model (3). Since the main development of the models (2), (3) and (9), beyond the one step model, is the admission of intermediate energetic species, it would seem equally valid to consider the simpler models (2) or (9).

Moreover, disturbances from an equilibrium state involving both forward and reverse reactions (as in real hydrocarbon chemistry at high temperature) generally evolve according to a local linearization, at least to leading order for small disturbances. Since the mass fraction $Y_{\mathrm{Z}}$ of $\mathrm{Z}$ is zero at chemical equilibrium, one should therefore expect small deviations from $Y_{Z}=0$ to evolve in proportion to $Y_{\mathrm{Z}}$, for realistic modelling of hydrocarbon oxidation. This is the case for the models (2) and (9) but not the model (3). For this purpose, not only is $Z$ thought of as representing some kind of 'pool' of intermediate reactants, it also measures deviation from 
chemical equilibrium. Because the 'pool' is depleted at equilibrium, it is useful to think of $\mathrm{Z}$ as representing only the fraction of intermediates that are available for conversion into products and heat. In this context, the models (2) and (9) should actually provide a better representation of hydrocarbon and hydrogen oxidation than the model (3), indirectly including the effects of reverse reaction.

Taking the chain-branching reaction to generate no heat and to have a large activation temperature, the kinetic model (2) has recently been used to describe planar flames and flame balls [28, 29] in the limit as $T_{\mathrm{B}} \rightarrow \infty$. The same model was used in [30] to describe the stability of flame balls. These studies found that multiple branches of solution arise under nonadiabatic conditions, as is found using one step chemical models although there are significant differences in the detail. Moreover the leading order asymptotic solutions remain remarkably close to accurate numerical solutions based on the model (2) even at realistic values of $T_{\mathrm{B}} \approx 8500 \mathrm{~K}[28]$.

As well as considering varying degrees of chain-branching, through the model (9), the basis of the kinetic model and its properties have been examined in greater depth in this article than in [28-30]. The branching reaction is still taken to generate no heat; a second order description of an inner structure where branching chemistry predominates is then fairly straightforward, leading to a flame-sheet model with essentially linear jump conditions, to leading order, across a reaction interface. The leading order form of the flame-sheet model is used to examine the structure and stability of planar premixed flames, although second order accuracy of the flame structure is recovered when solutions are interpreted in the manner shown in the Appendix.

In cases where the concentration of $\mathrm{Z}$ is small and essentially non-zero in only a relatively small part of the overall flame structure it has been found that a further asymptotic reduction leads to a model that bears some notable similarity to the one-step model $[28,30]$. In fact it is possible to identify an effective Zel'dovich number for a one-step model that would provide the same solutions, to leading order, with the same stability properties. The structure and stability of flames described in this way are examined in detail, highlighting both the technical similarities to the one-step model and the differences that remain in their interpretation.

\section{Model equations with chain branching kinetics}

Taking the rates of the reactions in the kinetic scheme (9) to be determined by elementary kinetics, appropriate reactive diffusive conservation equations are

$$
\begin{gathered}
\rho\left(\partial_{t}+\mathbf{u} \cdot \nabla\right) Y_{\mathrm{F}}=\rho D_{\mathrm{F}} \nabla^{2} Y_{\mathrm{F}}-\mu_{\mathrm{F}} W_{\mathrm{F}} \omega_{\mathrm{B}} \\
\rho\left(\partial_{t}+\mathbf{u} \cdot \nabla\right) Y_{\mathrm{Z}}=\rho D_{\mathrm{Z}} \nabla^{2} Y_{\mathrm{Z}}+\mu_{\mathrm{Z}} W_{\mathrm{Z}} \omega_{\mathrm{B}}-W_{\mathrm{Z}} \omega_{\mathrm{C}} \\
\rho C_{\mathrm{p}}\left(\partial_{t}+\mathbf{u} \cdot \nabla\right) T=\lambda \nabla^{2} T+Q \omega_{\mathrm{C}}-\ell \\
\omega_{\mathrm{B}}=A_{\mathrm{B}} \frac{\rho Y_{\mathrm{F}}}{W_{\mathrm{F}}} \frac{\rho Y_{\mathrm{Z}}}{W_{\mathrm{Z}}} \mathrm{e}^{-T_{\mathrm{B}} / T} \quad \omega_{\mathrm{C}}=A_{\mathrm{C}} \frac{\rho Y_{\mathrm{Z}}}{W_{\mathrm{Z}}} \frac{\rho}{W}
\end{gathered}
$$

where $Q$ is the energy released in converting a mole of the intermediate species into products. The molecular weights of the fuel and intermediate species are denoted by $W_{\mathrm{F}}$ and $W_{\mathrm{Z}}$, respectively, and $A_{\mathrm{B}}$ and $A_{\mathrm{C}}$ are the pre-exponential factors in the Arrhenius rate laws for the branching and completion reactions.

In these equations, the mass fraction of the fuel $Y_{\mathrm{F}}(t, \mathbf{x})$, the mass fraction of the intermediate reactant $Y_{\mathrm{Z}}(t, \mathbf{x})$ and the absolute temperature $T(t, \mathbf{x})$ are functions of time $t$ and position $\mathbf{x}$. For simplicity, the density $\rho$, diffusion coefficients $D_{\mathrm{F}}$ and $D_{\mathrm{Z}}$ of fuel and intermediate, respectively, the mean molecular weight $W$, the specific heat $C_{\mathrm{p}}$ and the thermal conductivity $\lambda$ 
are all taken to be constant. The fluid velocity is $\mathbf{u}$ and $\ell$ represents the rate of heat loss to infinity by radiation. For a planar flame travelling along an $x$-axis from large positive values of $x$, suitable 'unburnt' or 'cold' boundary conditions are

$$
\lim _{x \rightarrow-\infty}\left(Y_{\mathrm{F}}, Y_{\mathrm{Z}}, T\right)=\left(Y_{\mathrm{Fc}}, 0, T_{\mathrm{c}}\right)
$$

It is informative briefly to examine three particular aspects of this model.

\subsection{Adiabatic flame temperature}

Suppose that the system is adiabatic (so that $\ell=0$ ) and that a reaction wave of constant speed $S$ travels in the negative $x$-direction. Then steady solutions in a reference frame that travels with the wave do not vary with $t, y$ and $z$. Only derivatives with respect to $x$ remain non-zero, leading to the equations

$$
\begin{aligned}
\rho S Y_{\mathrm{F} x} & =\rho D_{\mathrm{F}} Y_{\mathrm{F} x x}-\mu_{\mathrm{F}} W_{\mathrm{F}} \omega_{\mathrm{B}} \\
\rho S Y_{\mathrm{Z} x} & =\rho D_{\mathrm{Z}} Y_{\mathrm{Z} x x}+\mu_{\mathrm{Z}} W_{\mathrm{Z}} \omega_{\mathrm{B}}-W_{\mathrm{Z}} \omega_{\mathrm{C}} \\
\rho C_{\mathrm{p}} S T_{x} & =\lambda T_{x x}+Q \omega_{\mathrm{C}}
\end{aligned}
$$

with the 'cold boundary' conditions (11). Eliminating $\omega_{\mathrm{B}}$ and $\omega_{\mathrm{C}}$ and integrating once gives the specific enthalpy as

$$
\begin{aligned}
h & =C_{\mathrm{p}} T+\frac{\mu_{\mathrm{Z}} Q}{\mu_{\mathrm{F}} W_{\mathrm{F}}} Y_{\mathrm{F}}+\frac{Q}{W_{\mathrm{Z}}} Y_{\mathrm{Z}} \\
& =C_{\mathrm{p}} T_{\mathrm{c}}+\frac{\mu_{\mathrm{Z}} Q}{\mu_{\mathrm{F}} W_{\mathrm{F}}} Y_{\mathrm{Fc}}+\frac{\lambda}{\rho S} T_{x}+\frac{\mu_{\mathrm{Z}} Q}{\mu_{\mathrm{F}} W_{\mathrm{F}}} \frac{D_{\mathrm{F}}}{S} Y_{\mathrm{F} x}+\frac{Q}{W_{\mathrm{Z}}} \frac{D_{\mathrm{Z}}}{S} Y_{\mathrm{Z} x} .
\end{aligned}
$$

Setting all derivatives and both fuel and intermediate mass fractions to zero, representing a uniform adiabatic state with complete chemical conversion, the adiabatic flame temperature (or burnt-gas temperature) can now be identified as

$$
T_{\mathrm{b}}=T_{\mathrm{c}}+\frac{\mu_{\mathrm{Z}} Q}{\mu_{\mathrm{F}} C_{\mathrm{p}} W_{\mathrm{F}}} Y_{\mathrm{Fc}} \quad \text { with } \quad Y_{\mathrm{F}}=Y_{\mathrm{Z}}=Y_{\mathrm{F} x}=Y_{\mathrm{Z} x}=T_{x}=0
$$

\subsection{Crossover temperature}

In a spatially uniform system the mass fraction of the intermediate $\mathrm{Z}$ satisfies

$$
\frac{\mathrm{d} Y_{\mathrm{Z}}}{\mathrm{d} t}=\rho Y_{\mathrm{Z}}\left(\frac{\mu_{\mathrm{Z}} A_{\mathrm{B}}}{W_{\mathrm{F}}} Y_{\mathrm{F}} \mathrm{e}^{-T_{\mathrm{B}} / T}-\frac{A_{\mathrm{C}}}{W}\right)
$$

the solutions of which depend very strongly on the sign of the right hand side. An equilibrium at $Y_{\mathrm{Z}}=0$ is unstable if the right hand side is positive for small positive values of $Y_{\mathrm{Z}}$ and is stable if the right side is negative. This identifies a 'crossover temperature' $T_{\times}$at which

$$
Y_{\mathrm{Fc}} \mathrm{e}^{-T_{\mathrm{B}} / T_{\times}}=\frac{W_{\mathrm{F}} A_{\mathrm{C}}}{\mu_{\mathrm{Z}} W A_{\mathrm{B}}} .
$$

At temperatures below $T_{\times}$, with $Y_{\mathrm{F}}=Y_{\mathrm{Fc}}$, any small concentration of intermediates decreases with time towards zero. However, at any fixed temperature above $T_{\times}$a small concentration of intermediates increases exponentially with time until the mass fraction $Y_{\mathrm{F}}$ decreases sufficiently to reverse the sign of the right side of (14). 
It is interesting to note that the two-step kinetic model (2) does not suffer from the so called 'cold boundary difficulty' of the one-step model for unburnt temperatures $T_{\mathrm{c}}$ below the crossover temperature $T_{\times}$. This is because the unburnt mixture is then in a stable state of chemical equilibrium with $Y_{\mathrm{Z}}=0$. Analogous arguments show that the model (3) does suffer from the cold boundary difficulty in the sense that the equilibrium $Y_{Z}=0$ is then always unstable at any temperature, although there would be no chemical activity in the complete absence of intermediates.

\subsection{Chain branching with diffusive removal of intermediates}

Now focusing on the full conservation equation for intermediate production and consumption, we have

$$
\left(\partial_{t}+\mathbf{u} \cdot \nabla\right) Y_{\mathrm{Z}}=\rho Y_{\mathrm{Z}}\left(\frac{\mu_{\mathrm{Z}} A_{\mathrm{B}}}{W_{\mathrm{F}}} Y_{\mathrm{F}} \mathrm{e}^{-T_{\mathrm{B}} / T}-\frac{A_{\mathrm{C}}}{W}\right)+D_{\mathrm{Z}} \nabla^{2} Y_{\mathrm{Z}}
$$

the right hand side of which differs from (14) through the presence of the Laplacian of $Y_{\mathrm{Z}}$. Intermediates will be produced by the branching reaction, consumed by the completion reaction and diffused by the Laplacian term. Generally speaking, the maximum concentration of intermediates arises where they are produced most strongly so that, at this point, the Laplacian of $Y_{Z}$ should be negative. Hence, in a spatially varying system, the latter term removes intermediates, making it more difficult to achieve chain branching. Temperatures around the crossover temperature $T_{\times}$are no longer enough. Instead let us attempt to identify another reference temperature $T_{\mathrm{r}}$ and make some order of magnitude estimates based on assuming that significant chain branching reactivity takes place around the temperature $T_{\mathrm{r}}$, using figure 1 as a guide.

It is useful to define $\theta=T_{\mathrm{B}} / T_{\mathrm{r}}$ and consider this to be a large parameter, since the activation temperature $T_{\mathrm{B}}$ is relatively large. It can then be noted that a temperature increase that is much above $T_{\mathrm{r}} \times\left(1+\mathrm{O}\left(\theta^{-1}\right)\right)$ causes the factor $\mathrm{e}^{-T_{\mathrm{B}} / T}$ to increase considerably, compared with its value at $T_{\mathrm{r}}$. In order for a balance to be retained in equation (16) we must therefore expect $Y_{\mathrm{F}}$ to decrease to very small values; in fact the reaction must stop because fuel is then rapidly consumed. On the other hand, for temperatures much below $T_{\mathrm{r}} \times\left(1-\mathrm{O}\left(\theta^{-1}\right)\right)$, the Arrhenius rate constant $k_{\mathrm{B}}$ becomes very small. Thus the branching reaction is only likely to be significant in a relatively narrow range of temperatures around $T_{\mathrm{r}}$, namey where $T-T_{\mathrm{r}}=\mathrm{O}\left(T_{\mathrm{r}} / \theta\right)$.

Outside this range, the conservation equation for intermediates has the approximate form

$$
\left(\partial_{t}+\mathbf{u} \cdot \nabla\right) Y_{\mathrm{Z}} \approx D_{\mathrm{Z}} \nabla^{2} Y_{\mathrm{Z}}-\rho \frac{A_{\mathrm{C}}}{W} Y_{\mathrm{Z}}
$$

with the first term on the right hand side representing diffusion of intermediates and the second representing the rate of their chemical conversion into products and heat. A natural length scale $r_{\mathrm{s}}$ for intermediate consumption and diffusion is therefore arrived at by balancing the terms on the right of this equation, giving

$$
r_{\mathrm{s}}^{2} \approx \frac{D_{\mathrm{Z}} W}{\rho A_{\mathrm{C}}}
$$

Because the completion reaction also generates heat, which has a diffusivity $\lambda /\left(\rho C_{\mathrm{p}}\right)$ that should be of the same order of magnitude as the diffusivity of the intermediates $D_{\mathrm{Z}}$, temperatures can be expected to change by the order of $T_{\mathrm{r}}$ over a similar length scale.

The length scale of the region in which chain branching chemistry is active, where temperatures change by about $T_{\mathrm{r}} / \theta$, should therefore be approximately $r_{\mathrm{s}} / \theta$. Likewise, anticipating that $Y_{\mathrm{F}}$ changes by the order of $Y_{\mathrm{Fc}}$ over the length scale $r_{\mathrm{s}}$, and that it is fully consumed by the 
branching reaction, we would expect to find $Y_{\mathrm{F}} \approx Y_{\mathrm{Fc}} / \theta$ where the branching reaction is active in equation (16). Because the intermediate concentration must be highest where intermediates are produced, values of $Y_{\mathrm{Z}}$ will only change by an amount of order $Y_{\mathrm{Z}} / \theta$ in the same region.

The dominant terms in this region must be the branching reaction rate and the diffusion term in equation (16), which therefore lead to the balance

$$
\rho Y_{\mathrm{Z}} \frac{\mu_{\mathrm{Z}} A_{\mathrm{B}}}{W_{\mathrm{F}}} Y_{\mathrm{F}} \mathrm{e}^{-T_{\mathrm{B}} / T} \approx \rho Y_{\mathrm{Z}} \frac{\mu_{\mathrm{Z}} A_{\mathrm{B}}}{W_{\mathrm{F}}} \frac{Y_{\mathrm{Fc}}}{\theta} \mathrm{e}^{-T_{\mathrm{B}} / T_{\mathrm{r}}} \approx-D_{\mathrm{Z}} \nabla^{2} Y_{\mathrm{Z}} \approx D_{\mathrm{Z}} \frac{Y_{\mathrm{Z}} / \theta}{\left(r_{\mathrm{s}} / \theta\right)^{2}}
$$

or, after using (17)

$$
Y_{\mathrm{Fc}} \mathrm{e}^{-T_{\mathrm{B}} / T_{\mathrm{r}}} \approx \theta^{2} \frac{W_{\mathrm{F}} A_{\mathrm{C}}}{\mu_{\mathrm{Z}} W A_{\mathrm{B}}} .
$$

Using equation (15) this can be expressed as

$$
\mathrm{e}^{-T_{\mathrm{B}} / T_{\mathrm{r}}} \approx \theta^{2} \mathrm{e}^{-T_{\mathrm{B}} / T_{\times}} \quad \text { or } \quad \frac{T_{\mathrm{B}}}{T_{\times}}-\frac{T_{\mathrm{B}}}{T_{\mathrm{r}}} \approx 2 \ln \frac{T_{\mathrm{B}}}{T_{\mathrm{r}}} .
$$

For values of $T_{\mathrm{B}}$ that are much larger than the temperature $T_{\mathrm{r}}$, about which chain branching is able to compete with the diffusive removal of intermediates, this relation shows that $T_{\mathrm{r}}$ must exceed the crossover temperature $T_{\times}$by about $T_{\times} \theta^{-1} \ln \theta^{2}$. Solving equation (19) for $T_{\mathrm{r}}$ as a function of $T_{\mathrm{B}}$, for different values of $T_{\times}$, leads to the variations plotted in figure 2 (showing both $T_{\mathrm{r}}$ and $\theta$ ). For an activation temperature of $T_{\mathrm{B}}=8460 \mathrm{~K}$ with $T_{\times} \approx 1200 \mathrm{~K}$, which seems
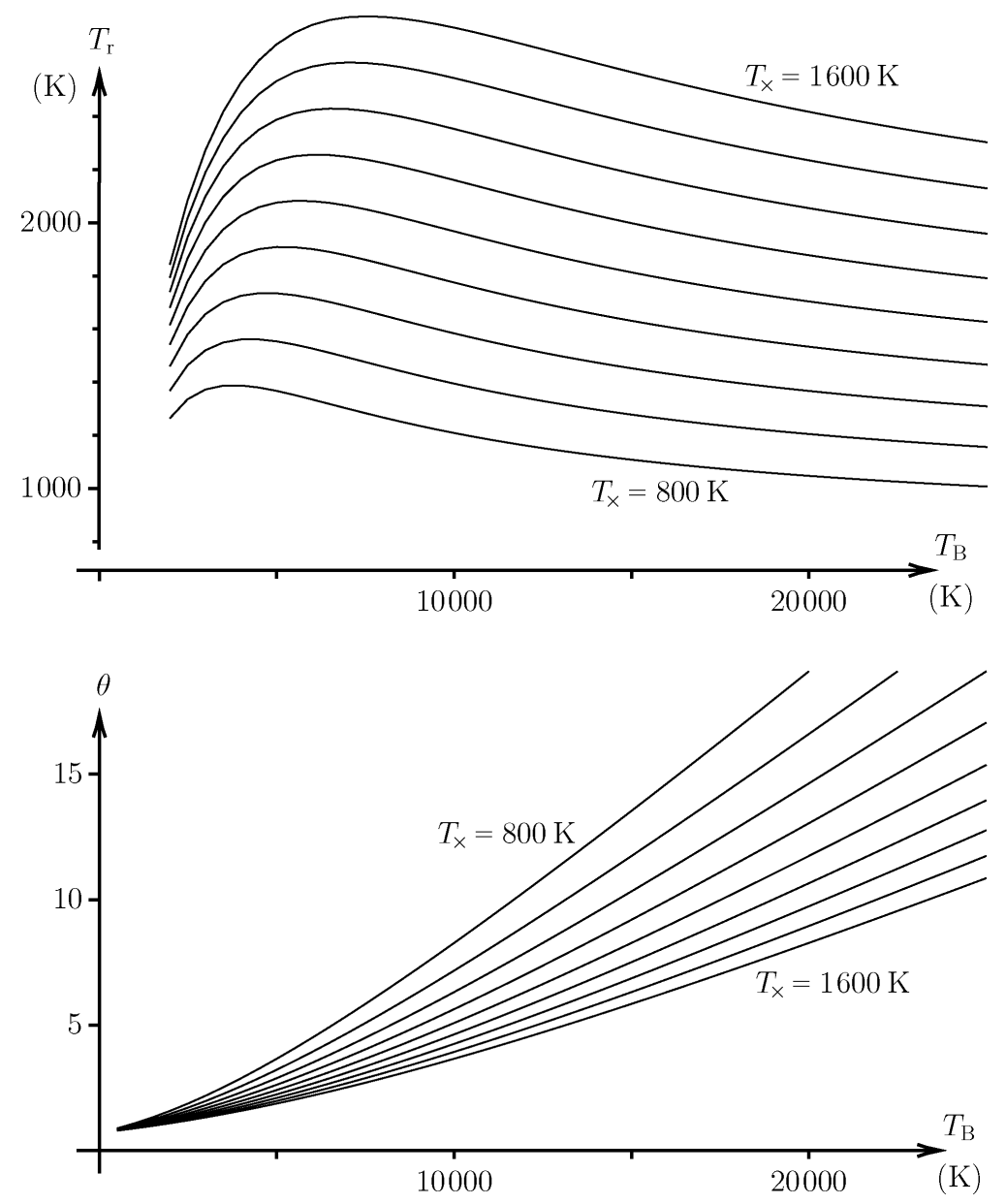

Figure 2. The reference temperature $T_{\mathrm{r}}$ and the dimensionless activation energy $\theta$ as functions of the activation temperature $T_{\mathrm{B}}$ at fixed values of the crossover temperature $T_{\times}$increasing from $800 \mathrm{~K}$ to $1600 \mathrm{~K}$ in steps of $100 \mathrm{~K}$. 
to be illustrative of hydrocarbon oxidation, it is found that $T_{\mathrm{r}} \approx 2000 \mathrm{~K}$ and $\theta \approx 4.2$. The values become $T_{\mathrm{r}} \approx 1450 \mathrm{~K}$ and $\theta \approx 5.9$ for $T_{\times} \approx 900 \mathrm{~K}$, illustrating hydrogen oxidation.

Finally, the branching reaction both creates the intermediate $\mathrm{Z}$ and consumes the fuel $\mathrm{F}$ in a relatively narrow layer. Within this layer, the branching reaction terms containing $\omega_{\mathrm{B}}$ and the diffusive terms containing $\nabla^{2} Y_{\mathrm{F}}$ and $\nabla^{2} Y_{\mathrm{Z}}$ are very large in the first two equations of (10), requiring the dominant balances

$$
\frac{D_{\mathrm{F}}}{\mu_{\mathrm{F}} W_{\mathrm{F}}} \nabla^{2} Y_{\mathrm{F}} \approx \frac{\omega_{\mathrm{B}}}{\rho} \approx-\frac{D_{\mathrm{Z}}}{\mu_{\mathrm{Z}} W_{\mathrm{Z}}} \nabla^{2} Y_{\mathrm{Z}}
$$

This relationship and those already discussed are useful in setting up a dimensionless version of the governing model equations.

\subsection{Dimensionless form of the model}

It is now convenient to introduce the following rescaling of the variables in the problem

$$
t=t_{\mathrm{s}} t^{\prime}, \quad \mathbf{x}=r_{\mathrm{s}} \mathbf{x}^{\prime}, \quad T=T_{\mathrm{s}} T^{\prime}, \quad Y_{\mathrm{F}}=Y_{\mathrm{Fc}} F, \quad Y_{\mathrm{Z}}=Y_{\mathrm{Zs}} Z
$$

along with the definitions

$$
\begin{aligned}
& r_{\mathrm{s}}^{2}=\frac{D_{\mathrm{Z}} W}{\rho A_{\mathrm{C}}}, \quad t_{\mathrm{s}}=\frac{\rho C_{\mathrm{p}}}{\lambda} r_{\mathrm{s}}^{2}, \quad \mathbf{u}^{\prime}=\frac{t_{\mathrm{s}}}{r_{\mathrm{s}}} \mathbf{u}, \quad \ell^{\prime}=\frac{\ell t_{\mathrm{s}}}{\rho C_{\mathrm{p}} T_{\mathrm{s}}}, \quad T_{\mathrm{c}}=T_{\mathrm{s}} T_{\mathrm{c}}^{\prime} \\
& \theta=\frac{T_{\mathrm{B}}}{T_{\mathrm{s}}}, \quad \theta^{2} \mathrm{e}^{T_{\mathrm{B}} / T_{\mathrm{r}}}=\frac{\mu_{\mathrm{Z}} W A_{\mathrm{B}}}{W_{\mathrm{F}} A_{\mathrm{C}}} Y_{\mathrm{Fc}}, \quad Q^{\prime}=\frac{\mu_{\mathrm{Z}} Q Y_{\mathrm{Fc}} / W_{\mathrm{F}}}{\mu_{\mathrm{F}} C_{\mathrm{p}}\left(T_{\mathrm{s}}-T_{\mathrm{c}}\right)}, \quad T_{\mathrm{b}}=T_{\mathrm{s}} T_{\mathrm{b}}^{\prime} \\
& \mathrm{Le}_{\mathrm{F}}=\frac{\lambda}{\rho C_{\mathrm{p}} D_{\mathrm{F}}}, \quad \mathrm{Le}_{\mathrm{Z}}=\frac{\lambda}{\rho C_{\mathrm{p}} D_{\mathrm{Z}}}, \quad Y_{\mathrm{Zs}}=\frac{D_{\mathrm{F}} \mu_{\mathrm{Z}} W_{\mathrm{Z}}}{D_{\mathrm{Z}} \mu_{\mathrm{F}} W_{\mathrm{F}}} Y_{\mathrm{Fc}}, \quad T_{\times}=T_{\mathrm{s}} T_{\times}^{\prime}
\end{aligned}
$$

which make use of the relations (13), (17), (18) and (20) identified earlier, with the scaling temperature $T_{\mathrm{s}}$ replacing the reference temperature $T_{\mathrm{r}}$ in the definition of $\theta$. In the following analysis $T_{\mathrm{s}}$ will be taken to be equal to $T_{\mathrm{r}}$, although other choices may sometimes be more useful (as shown in the Appendix).

After substituting into the model equations (10) and the boundary conditions (11), and dropping the primes, the equations to be satisfied become (for $T_{\mathrm{s}}=T_{\mathrm{r}}$ )

$$
\begin{aligned}
\operatorname{Le}_{\mathrm{F}}\left(\partial_{t}+\mathbf{u} \cdot \nabla\right) F & =\nabla^{2} F-\theta^{2} F Z k(T) \\
\operatorname{Le}_{\mathrm{Z}}\left(\partial_{t}+\mathbf{u} \cdot \nabla\right) Z & =\nabla^{2} Z+\theta^{2} F Z k(T)-Z \\
\left(\partial_{t}+\mathbf{u} \cdot \nabla\right) T & =\nabla^{2} T+\frac{1-T_{\mathrm{c}}}{\operatorname{Le}_{\mathrm{F}}} Q Z-\ell \\
k(T) & =\exp (\theta-\theta / T)
\end{aligned}
$$

with the upstream boundary conditions for a flame travelling from the right

$$
\lim _{x \rightarrow-\infty}(F, Z, T)=\left(1,0, T_{\mathrm{c}}\right) .
$$

Along the same lines as (13) the dimensionless adiabatic flame temperature for this system of equations can be shown to be $T_{\mathrm{b}}=T_{\mathrm{c}}+\left(1-T_{\mathrm{c}}\right) Q$, in which the cold-gas temperature $T_{\mathrm{c}}$ should be less than one. The dimensionless reference temperature $T_{\mathrm{r}}^{\prime}$ becomes precisely one if we set $T_{\mathrm{s}}=T_{\mathrm{r}}$ and flame temperatures should normally exceed this level, so that values of $Q$ should normally exceed unity for a flame to be possible. 


\section{Asymptotic structure of the branching reaction}

As in the dimensional form of the model equations, a dimensionless crossover temperature $T_{\times}$ arises when the rates of the branching and completion reactions in the equation for $Z$ are the same (taking $F=1$, as in the cold unburnt gas). That is

$$
\theta^{2} \exp \left(\theta-\theta / T_{\times}\right)=1 \quad \text { or } \quad T_{\times}=\left(1+\theta^{-1} \ln \theta^{2}\right)^{-1} \sim 1-2 \theta^{-1} \ln \theta
$$

for large values of $\theta$. Below this temperature, no branching at all is possible and above this temperature branching can proceed in a homogeneous mixture having the value of $F$ found at $x=-\infty$, for any initial positive value of $Z$. As discussed earlier, the inhomogeneous branching that arises in a premixed flame requires a different form of balance where diffusive processes and the branching reaction are balanced in a narrow layer where the dimensionless temperature $T$ is closer to the reference temperature of $T_{\mathrm{r}}^{\prime}=1$.

This balance can be examined in more detail if $\theta$ is large. The branching reaction can then be taken to occur in a narrow region around a surface where $\mathbf{x}=\mathbf{R}(t, y, z)$. Two different forms of asymptotic structure arise, depending on whether the temperature increases through the region of branching reaction or it has a maximum value where the branching reaction occurs. In the former case the rate constant $k(T)$ increases unboundedly as temperature increases and the fuel $F$ must be completely consumed. In the latter case the rate constant $k(T)$ falls to zero as temperature decreases on either side, allowing fuel to leak through the region of branching.

\subsection{Temperature increasing through the region of branching reaction}

Scaled coordinates that describe the inner structure of the branching chemistry, when temperature has a non-zero gradient, can be defined as follows

$$
\begin{aligned}
& \mathbf{x}=\mathbf{R}+\widehat{\mathbf{n}} \theta^{-1} \eta \quad T \sim 1+\theta^{-1} s(\eta, t, \mathbf{R}), \\
& Z \sim \bar{Z}+\theta^{-1} \zeta(\eta, t, \mathbf{R}) \quad F \sim \theta^{-1} f(\eta, t, \mathbf{R})
\end{aligned}
$$

where $\widehat{\mathbf{n}}$ is a normal direction, so that $\eta$ measures small distances, of order $\theta^{-1}$, at right angles to the interface. The precise location of the interface $\mathbf{R}$ and the value of $\bar{Z}(t, \mathbf{R})$ remain to be defined.

The functions $s, \zeta$ and $f$ measure relatively small changes in temperature, intermediate and fuel concentrations. Their variation with time $t$ and position on the interface $\mathbf{R}$, is slow in comparison to their dependence on the 'short' normal distance $\eta$. Substituted into the equations (23) they are found to satisfy the equations

$$
f_{\eta \eta}=\bar{Z} f \mathrm{e}^{s}=-\zeta_{\eta \eta}, \quad s_{\eta \eta}=0 .
$$

when all higher-order effects are neglected as $\theta \rightarrow \infty$. The equations $f_{\eta \eta}+\zeta_{\eta \eta}=0$ and $s_{\eta \eta}=0$ can each be integrated twice, giving

$$
f+\zeta=v \eta, \quad s=s_{0}+\mu \eta
$$

in which only three constants of integration appear. One constant in the first equation has been set to zero by, implicitly, choosing the value of $\bar{Z}$ so as to set the origin of $\zeta$ appropriately. The temperature gradient through the reaction interface is given by $\mu$ which is taken to be non-zero.

The variable $\eta$ can now be eliminated, in favour of $s$, to give either

$$
\mu^{2} f_{s s}=\bar{Z} f \mathrm{e}^{s} \quad \text { or } \quad \xi f_{\xi \xi}+f_{\xi}-\xi f=0 \quad \text { with } \quad \xi=2 \mathrm{e}^{s / 2} \bar{Z}^{1 / 2} /|\mu|
$$


in which the definition of the variable $\xi$ uncovers a linear equation (modified Bessel's equation of order zero) to be solved for $f$ with $\xi>0$. Only the $\mathrm{K}_{0}(\xi)$ solution, and not $\mathrm{I}_{0}(\xi)$, involves $f$ tending to zero, as it must do, for large values of $\xi$ (or $s$ ). Thus the solution can be written as

$$
\begin{aligned}
f & =A \mathrm{~K}_{0}(\xi)=A \mathrm{~K}_{0}\left(2 \mathrm{e}^{s / 2} \bar{Z}^{1 / 2} /|\mu|\right) \\
& =A \times \begin{cases}\mathrm{O}\left(\mathrm{e}^{-s / 2} \exp \left(-2 \mathrm{e}^{s / 2} \bar{Z}^{1 / 2} /|\mu|\right)\right) & \text { as } s \rightarrow \infty \\
-\frac{1}{2} s-\gamma+\ln \left(|\mu| / \bar{Z}^{1 / 2}\right)+\mathrm{O}\left(s \mathrm{e}^{s}\right) & \text { as } s \rightarrow-\infty\end{cases}
\end{aligned}
$$

in which $\gamma$ is Euler's constant $(\gamma \approx 0.5772 \cdots)$ and $A$ is an arbitrary positive constant (so that $f>0$ ). The asymptotic behaviour [31] of $f$ is also shown for large positive and negative values of $s$. It follows that

$$
f=v \eta-\zeta \sim A \times \begin{cases}0 & \text { as } \mu \eta \rightarrow \infty \\ -\frac{1}{2}\left(s_{0}+\mu \eta\right)-\gamma+\ln \left(|\mu| / \bar{Z}^{1 / 2}\right) & \text { as } \mu \eta \rightarrow-\infty .\end{cases}
$$

That is, both the fuel variable $f$ and the intermediate variable $\zeta$ asymptote towards straight lines, as seen from far away from the region of branching chemistry (in terms of $\eta$ ) with $f$ approaching zero on the hotter side. The location of the interface $\mathbf{x}=\mathbf{R}$, or $\eta=0$, can now be made more precise by defining it to lie where these two lines intersect. As a result the asymptotes become

$$
f \sim A \times\left\{\begin{array}{ll}
0 & \text { as } \mu \eta \rightarrow \infty \\
-\frac{1}{2} \mu \eta & \text { as } \mu \eta \rightarrow-\infty
\end{array} \quad \zeta \sim A \times \begin{cases}\nu \eta & \text { as } \mu \eta \rightarrow \infty \\
\left(\nu+\frac{1}{2} \mu\right) \eta & \text { as } \mu \eta \rightarrow-\infty\end{cases}\right.
$$

along with the condition that

$$
s_{0}=\ln \left(\mu^{2} / \bar{Z}\right)-2 \gamma
$$

which determines the value of the constant $s_{0}$. The other constants, namely $\mu, v, A$ and $\bar{Z}$ are all determined by matching with asymptotic solutions for an outer region, from which the region of branching reaction is seen as an infinitesimally thin interface.

Treating $F, Z$ and $T$ as the outer variables (in order to avoid a plethora of notation) matching the values and slopes of each variable provides the following conditions at the interface, ignoring errors smaller than order $\theta^{-1}$

$F^{ \pm}=0, \quad Z^{ \pm}=\bar{Z}, \quad T^{ \pm}=1+\theta^{-1} s_{0}, \quad F_{n}^{+}-F_{n}^{-}=Z_{n}^{-}-Z_{n}^{+}=\frac{1}{2} A|\mu|, \quad T_{n}^{+}=T_{n}^{-}$ where $n$ measures normal distance from the interface $\left(n=\theta^{-1} \eta\right)$ and superscripts ${ }^{+}$and $^{-}$refer to one sided limits as $n \rightarrow 0^{+}$and $n \rightarrow 0^{-}$, respectively. The results are the same for either of the two possible directions in which the normal $\widehat{\mathbf{n}}$ can be chosen to point. By eliminating $\mu, A$ and $\bar{Z}$ the conditions can be written in the form

$$
\left[F_{n}\right]+\left[Z_{n}\right]=[Z]=\left[T_{n}\right]=F^{ \pm}=0, \quad T^{ \pm}=1+\theta^{-1}\left(\ln \left(T_{n}^{2} / Z\right)-2 \gamma\right)
$$

in which the brackets [ $\cdot]$ refer to the jump in value from $n=0^{-}$to $n=0^{+}$.

It can be noted that a choice for the scaling temperature $T_{\mathrm{s}}$ in (21) and (22) could have been made so as to set the dimensionless branching-sheet temperature $T^{ \pm}$to exactly unity. This would define $T_{\mathrm{s}}$ to be precisely the dimensional branching-sheet temperature. A dimensional equivalent of (28) then makes clear that $T_{\mathrm{S}}$ would depend weakly on the solution (through the value of $T_{n}^{2} / Z$ at the reaction sheet) as would some of the dimensionless parameters defined in (22). This matter is examined further in the Appendix.

In this analysis, the ratio $T_{n}^{2} / Z$, which is also to be evaluated at $n=0$, has been assumed to be positive and of order unity. Clearly it cannot approach infinity because there would be no 
branching chemistry if $Z=0$. As $T_{n}$ approaches zero the situation approaches that in which the temperature has a maximum value in the region of branching reaction.

\subsection{Maximum temperature in the region of branching reaction}

In situations where $T$ has a maximum value in the region of branching chemistry a new set of scaled variables is needed to describe the inner problem. A suitable redefinition of $\eta, f, \zeta$ and $s$ is

$$
\begin{array}{ll}
\mathbf{r}=\mathbf{R}+\widehat{\mathbf{n}} \theta^{-1 / 2} \eta & T \sim 1+\theta^{-1}\left(s(\eta, t, \mathbf{R})-\frac{3}{2} \ln \theta\right) \\
Z \sim \bar{Z}+\theta^{-1 / 2} \zeta(\eta, t, \mathbf{R}) & F \sim \bar{F}+\theta^{-1 / 2} f(\eta, t, \mathbf{R})
\end{array}
$$

in which a new parameter $\bar{F}$ has been introduced to take into account a possible order one leakage of fuel $F$ through the region. In order to obtain a suitable asymptotic balance of terms in the governing equations, the variables $\eta, f$ and $\zeta$ now measure changes on a larger scale (of order $\theta^{-1 / 2}$ rather than $\theta^{-1}$ ) and the origin of $s$ lies at a lower value of $T$, reduced by $\frac{3}{2} \theta^{-1} \ln \theta$. This origin is only slightly higher than the crossover temperature $T_{\times}$, given in (25), below which branching chemistry cannot be sustained. The parameters $\bar{F}, \bar{Z}$ and $\mathbf{R}$ remain to be defined more precisely.

Substituting the rescalings (29) into equations (23) and neglecting asymptotically small terms, as $\theta \rightarrow \infty$, gives

$$
f_{\eta \eta}=\bar{F} \bar{Z} \mathrm{e}^{s}=-\zeta_{\eta \eta}, \quad s_{\eta \eta}=\bar{\ell}-\bar{Q} \bar{Z}
$$

in which $\bar{\ell}$ represents the asymptotic limiting value of the heat-loss term $\ell$ in the inner branching region and, for brevity, we have defined $\bar{Q}=\left(1-T_{\mathrm{c}}\right) Q / \mathrm{Le}_{\mathrm{F}}$. In order for the temperature $s$ to have a maximum, the value of $\bar{\ell}-\bar{Q} \bar{Z}$ must be negative, requiring that $\bar{Q} \bar{Z}>\bar{\ell}$. Two equations can be integrated twice to give

$$
f+\zeta=v \eta, \quad s=s_{0}-\frac{1}{2}(\bar{Q} \bar{Z}-\bar{\ell}) \eta^{2}
$$

in which one constant of integration has been eliminated by defining $\bar{Z}$ appropriately and the location of the interface $\mathbf{R}$ has been defined so that the maximum value of $s$ is exactly at the interface $\eta=0$. The remaining equation for $f$ can be written as

$$
f_{\eta \eta}=\bar{F} \bar{Z} \mathrm{e}^{s_{0}} \mathrm{e}^{-(\bar{Q} \bar{Z}-\bar{\ell}) \eta^{2} / 2}
$$

and integrated twice to give

$$
\begin{aligned}
f=v \eta-\zeta= & \eta f_{\eta}^{-}+\bar{F} \bar{Z} \mathrm{e}^{s_{0}}\left(\frac{\pi / 2}{\bar{Q} \bar{Z}-\bar{\ell}}\right)^{1 / 2} \eta \operatorname{erfc}\left(-\eta \sqrt{\frac{1}{2}(\bar{Q} \bar{Z}-\bar{\ell})}\right) \\
& +\frac{\bar{F} \bar{Z} \mathrm{e}^{s_{0}}}{\bar{Q} \bar{Z}-\bar{\ell}} \exp \left(-(\bar{Q} \bar{Z}-\bar{\ell}) \eta^{2} / 2\right) \\
& \sim \begin{cases}\eta f_{\eta}^{-}+\bar{F} \bar{Z} \mathrm{e}^{s_{0}}\left(\frac{2 \pi}{\bar{Q} \bar{Z}-\bar{\ell}}\right)^{1 / 2} \eta & \text { as } \eta \rightarrow \infty \\
\eta f_{\eta}^{-} & \text {as } \eta \rightarrow-\infty\end{cases}
\end{aligned}
$$

with the constant of integration $f_{\eta}^{-}$representing the limiting value of $f_{\eta}$ as $\eta \rightarrow-\infty$. The other constant of integration has been set to zero by defining $\bar{F}$ appropriately. It can be seen that the straight line asymptotes for $f$ and $\zeta$, as $\eta \rightarrow \pm \infty$, all intersect at the interface $\eta=0$. This is a direct result of the choice made earlier for defining $\mathbf{R}$. 
The change in the value of $f_{\eta}$ from minus infinity to plus infinity can now be evaluated, giving

$$
\left[f_{\eta}\right]=f_{\eta}^{+}-f_{\eta}^{-}=\bar{F} \bar{Z} \mathrm{e}^{s_{0}}\left(\frac{2 \pi}{\bar{Q} \bar{Z}-\bar{\ell}}\right)^{1 / 2}
$$

where $f_{\eta}^{+}$is the limiting value of $f_{\eta}$ as $\eta \rightarrow \infty$. Hence we find that

$$
s_{0}=\ln \frac{\left[f_{\eta}\right]}{\bar{F} \bar{Z}}+\frac{1}{2} \ln \frac{\bar{Q} \bar{Z}-\bar{\ell}}{2 \pi} .
$$

Matching with the outer asymptotic problem, as before, now gives the jump conditions across the interface

$$
\begin{aligned}
{\left[F_{n}\right]+\left[Z_{n}\right] } & =[F]=[Z]=T_{n}^{ \pm}=0, \\
T^{ \pm} & =1+\theta^{-1}\left(\ln \frac{\left[F_{n}\right]}{F Z}+\frac{1}{2} \ln \frac{\left(1-T_{\mathrm{c}}\right) Q Z / \mathrm{Le}_{\mathrm{F}}-\ell}{2 \pi}-\frac{3}{2} \ln \theta\right)
\end{aligned}
$$

after neglecting terms that are smaller than order $\theta^{-1}$. As in the case when $T_{n} \neq 0$ at the interface, a different solution-dependent dimensional temperature scaling factor $T_{\mathrm{s}}$ could have been chosen so as to set $T^{ \pm}=1$. Also as before, the terms $F, Z$ and $\ell$ in the final equation are to be evaluated at the interface where, clearly, the value of $F$ must be positive. This shows that fuel leakage must occur if temperature has a maximum value at the interface.

\subsection{Reaction-sheet model}

In the limit as $\theta \rightarrow \infty$ the jump conditions (28) and (30) lose all dependence on $\theta$, to leading order. The same is true, to second order, if the scaling factor $T_{\mathrm{s}}$ is chosen so as to set $T^{ \pm}=1$, as discussed above. The problem can then be viewed as one in which the branching chemistry is confined to a thin sheet at $\mathbf{x}=\mathbf{R}$. Outside of this sheet the branching reaction-rate is zero so that the governing equations (23) become

$$
\begin{aligned}
\operatorname{Le}_{\mathrm{F}}\left(\partial_{t}+\mathbf{u} \cdot \nabla\right) F & =\nabla^{2} F \\
\operatorname{Le}_{\mathrm{Z}}\left(\partial_{t}+\mathbf{u} \cdot \nabla\right) Z & =\nabla^{2} Z-Z \\
\left(\partial_{t}+\mathbf{u} \cdot \nabla\right) T & =\nabla^{2} T+\frac{1-T_{\mathrm{c}}}{\operatorname{Le}_{\mathrm{F}}} Q Z-\ell .
\end{aligned}
$$

The two sets of jump conditions (28) and (30) can also then be written as the single set of conditions

$$
\left[F_{n}\right]+\left[Z_{n}\right]=\left[T_{n}\right]=[F]=[Z]=[T]=T-1=F T_{n}=0
$$

to be applied at the interface (where $T-1$ and $F T_{n}$ are to be evaluated). This requires that temperature $T$ takes the value one and that $F=0$ if the normal gradient $T_{n}$ is not equal to zero at the interface; if $T_{n}=0$ then the value of $F$ can be positive. Either way, the jump conditions (32) become purely linear, which is very useful mathematically. Another general requirement away from the interface is that $F$ must be zero wherever $T>1$.

If the nondimensionalization given in (21) and (22) is carried out with the scaling temperature $T_{\mathrm{s}}$ defined as $T_{\mathrm{s}}=T_{\mathrm{r}}$, then in cases where temperature increases through the interface errors of the order of $\theta^{-1}$ are incurred through setting $T=1$. If the maximum temperature lies at the interface, then errors are of the order of $\theta^{-1} \ln \theta$. However, if $T_{\mathrm{s}}$ is defined to be the dimensional value of the temperature at the interface, then the conditions (32) become more precise, involving errors that are only of order $\theta^{-2}$, but the dimensionless parameters of the 
problem, including $Q, T_{\mathrm{c}}$ and $\ell$, then depend on the solution. The latter definition of $T_{\mathrm{s}}$ is explored further in the Appendix.

\section{Structure of a premixed flame with linear heat loss}

If the velocity $\mathbf{u}$ is taken to have speed $S$, blowing in the $x$ direction, and if the rate of heat loss is modelled as $\ell=a\left(T-T_{\mathrm{c}}\right)$, with $a \geq 0$, then the reaction-sheet model equations (31) can be written as

$$
\begin{aligned}
\operatorname{Le}_{\mathrm{F}}\left(F_{t}+S F_{x}\right) & =\nabla^{2} F \\
\operatorname{Le}_{\mathrm{Z}}\left(Z_{t}+S Z_{x}\right) & =\nabla^{2} Z-Z \\
T_{t}+S T_{x} & =\nabla^{2} T+\frac{1-T_{\mathrm{c}}}{\operatorname{Le}_{\mathrm{F}}} Q Z-a\left(T-T_{\mathrm{c}}\right) .
\end{aligned}
$$

These equations are to be applied everywhere except at a reaction surface $\mathbf{x}=\mathbf{R}$ where the jump conditions (32) must be satisfied. The boundary conditions (24) describe the cold gas mixture for a flame that propagates from right to left.

\subsection{Steadily propagating planar flame}

Fuel and intermediate concentrations and temperature, for a steady planar flame propagating at a constant speed $S$, can be written in the form

$$
F=F_{0}(x), \quad Z=Z_{0}(x), \quad \frac{T-T_{\mathrm{c}}}{1-T_{\mathrm{c}}}=T_{0}(x)
$$

with the functions $F_{0}, Z_{0}$ and $T_{0}$ satisfying the differential equations (in which primes denote differentiation)

$$
\begin{aligned}
\mathrm{Le}_{\mathrm{F}} S F_{0}^{\prime} & =F_{0}^{\prime \prime} \\
\mathrm{Le}_{\mathrm{Z}} S Z_{0}^{\prime} & =Z_{0}^{\prime \prime}-Z_{0} \\
S T_{0}^{\prime} & =T_{0}^{\prime \prime}+\frac{Q}{\mathrm{Le}_{\mathrm{F}}} Z_{0}-a T_{0}
\end{aligned}
$$

except at $x=0$, which can be taken to be the position of the branching reaction-sheet. Jump conditions to be applied at $x=0$ are then

$$
\left[F_{0}^{\prime}\right]+\left[Z_{0}^{\prime}\right]=\left[T_{0}^{\prime}\right]=\left[F_{0}\right]=\left[Z_{0}\right]=\left[T_{0}\right]=T_{0}-1=F_{0} T_{0}^{\prime}=0
$$

and suitable boundary conditions as $x \rightarrow \pm \infty$ can be written as

$$
\lim _{x \rightarrow-\infty}\left(F_{0}, Z_{0}, T_{0}\right)=(1,0,0), \quad \lim _{x \rightarrow \infty}\left(F_{0}^{\prime}, Z_{0}, T_{0}^{\prime}\right)=(0,0,0) .
$$

In fact it is sufficient simply to require boundedness as $x \rightarrow \infty$.

Solving the equations (35) and applying all of the boundary and jump conditions at $x=0$, apart from $T_{0}-1=F_{0} T_{0}^{\prime}=0$, leads to the solutions

$$
F_{0}(x)=\left\{\begin{array}{ll}
\bar{F} & : x>0 \\
1-(1-\bar{F}) \mathrm{e}^{\mathrm{Le}_{\mathrm{F}} S x} & : x<0
\end{array} \quad Z_{0}(x)=\frac{\operatorname{Le}_{\mathrm{F}} S(1-\bar{F})}{\gamma_{1}+\gamma_{2}} \begin{cases}\mathrm{e}^{-\gamma_{2} x} & : x>0 \\
\mathrm{e}^{\gamma_{1} x} & : x<0\end{cases}\right.
$$


and

$$
\begin{array}{r}
T_{0}(x)=\frac{Q S(1-\bar{F})}{\mu_{1}+\mu_{2}} \begin{cases}\frac{\mathrm{e}^{-\mu_{2} x}}{\left(\gamma_{2}-\mu_{2}\right)\left(\gamma_{1}+\mu_{2}\right)} & : x>0 \\
\frac{\mathrm{e}^{\mu_{1} x}}{\left(\gamma_{1}-\mu_{1}\right)\left(\gamma_{2}+\mu_{1}\right)} & : x<0\end{cases} \\
-\frac{Q S(1-\bar{F})}{\gamma_{1}+\gamma_{2}} \begin{cases}\frac{\mathrm{e}^{-\gamma_{2} x}}{\left(\gamma_{2}-\mu_{2}\right)\left(\gamma_{2}+\mu_{1}\right)} & : x>0 \\
\frac{\mathrm{e}^{\gamma_{1} x}}{\left(\gamma_{1}-\mu_{1}\right)\left(\gamma_{1}+\mu_{2}\right)} & : x<0\end{cases}
\end{array}
$$

in which the constants $\gamma_{1}, \gamma_{2}, \mu_{1}$ and $\mu_{2}$ are defined as

$$
\gamma_{\{1,2\}}=\frac{1}{2}\left(\sqrt{4+\mathrm{Le}_{\mathrm{Z}}^{2} S^{2}} \pm \mathrm{Le}_{\mathrm{Z}} S\right), \quad \mu_{\{1,2\}}=\frac{1}{2}\left(\sqrt{4 a+S^{2}} \pm S\right)
$$

and the values of $\bar{F}$ and $S$ are not yet known. The derivative of temperature at $x=0$ is

$$
T_{0}^{\prime}(0)=\frac{Q S(1-\bar{F})\left(\gamma_{1} \mu_{1}-\gamma_{2} \mu_{2}\right)}{\left(\gamma_{1}+\gamma_{2}\right)\left(\mu_{1}+\mu_{2}\right)\left(\gamma_{1}+\mu_{2}\right)\left(\gamma_{2}+\mu_{1}\right)}
$$

Since $\gamma_{1}>\gamma_{2}>0$ and $\mu_{1}>\mu_{2} \geq 0$ for all values of $S>0$, Le $\mathrm{Z}>0$ and $a \geq 0$, this expression must be positive. As a result, we must have $\bar{F}=0$ in order to satisfy $F_{0} \bar{T}_{0}^{\prime}=0$ at $x=0$. The remaining condition, that $T_{0}(0)=1$, then leads to the formula

$$
Q S\left(\gamma_{1}+\gamma_{2}+\mu_{1}+\mu_{2}\right)=\left(\gamma_{1}+\gamma_{2}\right)\left(\mu_{1}+\mu_{2}\right)\left(\gamma_{1}+\mu_{2}\right)\left(\gamma_{2}+\mu_{1}\right)
$$

which, together with (38), becomes the algebraic relationship

$$
\begin{aligned}
2 Q S\left(\sqrt{4+\mathrm{Le}_{\mathrm{Z}}^{2} S^{2}}+\sqrt{4 a+S^{2}}\right)= & \sqrt{4+\mathrm{Le}_{\mathrm{Z}}^{2} S^{2}} \sqrt{4 a+S^{2}} \\
& \times\left(2(1+a)+\sqrt{4+\mathrm{Le}_{\mathrm{Z}}^{2} S^{2}} \sqrt{4 a+S^{2}}+\operatorname{Le}_{\mathrm{Z}} S^{2}\right)
\end{aligned}
$$

between $Q, S, \mathrm{Le}_{\mathrm{Z}}$ and $a$, which can be used to solve for any one parameter, given suitable values for the others.

Interestingly, the Lewis number of the fuel $\mathrm{Le}_{\mathrm{F}}$ does not appear in the relation (40), which means that the dimensionless flame-speed $S$ is independent of Le $\mathrm{F}_{\mathrm{F}}$. Even dimensionally, after using the scaling parameters defined in (22), the speed does not depend on the diffusivity of the fuel $D_{\mathrm{F}}$ in any way. However, the steady fuel concentration $F_{0}$ and the magnitude of the intermediate concentration $Z_{0}$ do depend on $\mathrm{Le}_{\mathrm{F}}$.

Solving equation (40) numerically leads to the flame-speeds $S$ shown in figures 3 and 4. Of course, solutions are only possible for heats of reaction $Q$ greater than unity because temperatures must exceed one for any branching chemistry to occur. In each case, with $Q>1$, there are two possible flame speeds for any positive value of the heat-loss parameter $a$ that is less than a maximum value $a_{\mathrm{m}}\left(Q, \mathrm{Le}_{\mathrm{Z}}\right)$. As $a$ decreases to zero, the lower flame-speed approaches zero while the upper speed approaches a maximum value $S_{\mathrm{m}}\left(Q, \mathrm{Le}_{\mathrm{Z}}\right)$ representing the adiabatic flame speed. As $a$ approaches $a_{\mathrm{m}}$ the two flame-speeds merge at a turning-point bifurcation in the curve of $S$ as a function of $a$. There are no solutions for larger values of $a$.

Figure 3 demonstrates that the curves of $S$ as a function of $a$ have a very similar structure over a wide range of values of heat of reaction and intermediate Lewis number, on scales that are normalized against the maximum values of $S$ and $a$. This point is further emphasized by the lower graph in figure 3 which shows that the ratio of the value of $S$ at the turning point, 

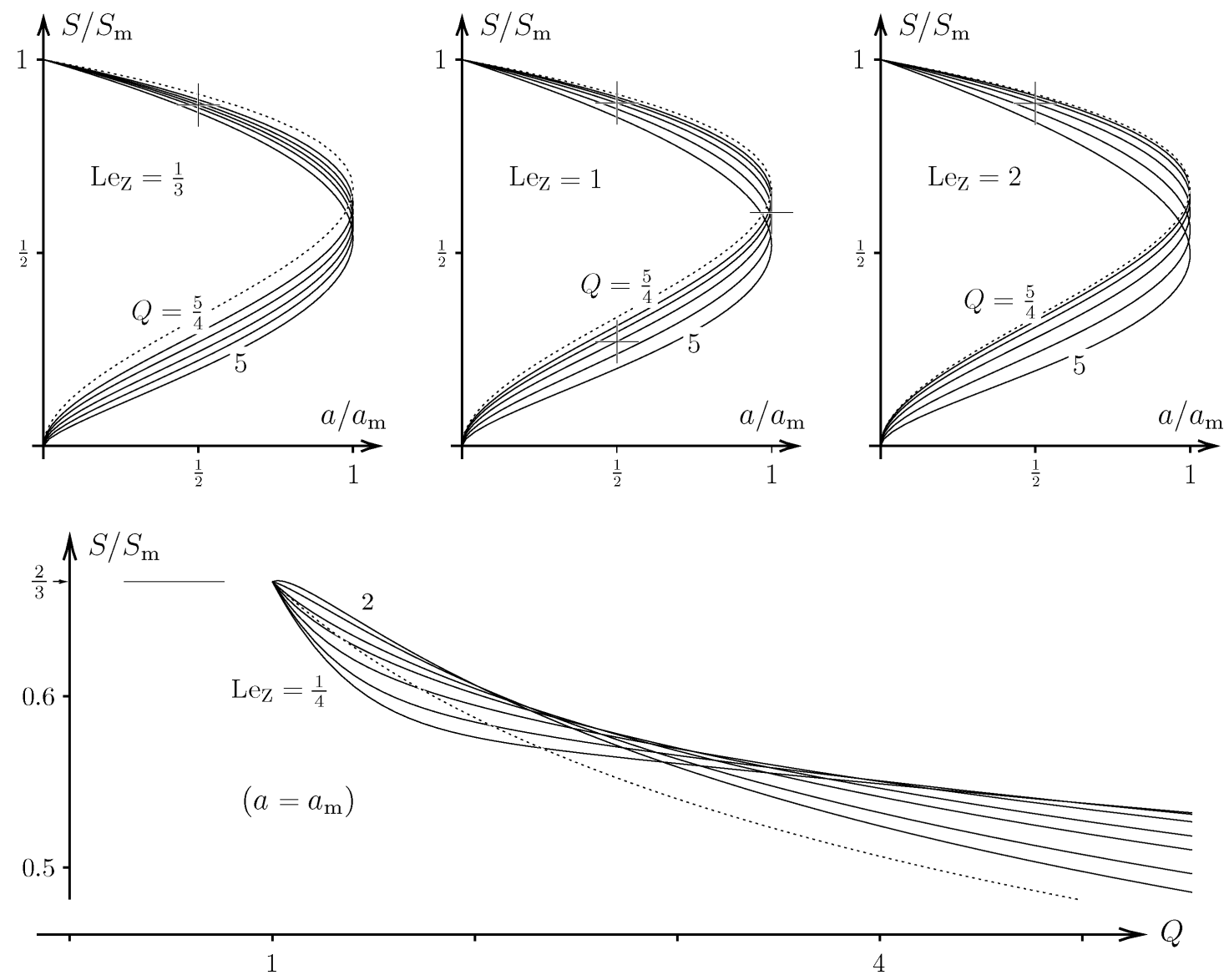

Figure 3. The upper graphs show the dimensionless flame-speed $S$ at any heat-loss parameter $a$ for the heats of reaction $Q=\frac{5}{4}, \frac{3}{2}, 2,3$ and 5, at fixed values of the intermediate Lewis number Le $\mathrm{Z}$, with values of $S$ and $a$ normalized against their maximum values $S_{\mathrm{m}}$ and $a_{\mathrm{m}}$; the dotted curves represent the formula $a \propto S^{2}\left(S_{\mathrm{m}}-S\right)$. In the lower graph the values of $S / S_{\mathrm{m}}$ that are found at the turning point (where $a=a_{\mathrm{m}}$ ) are plotted as a function of $Q$ for the values of the intermediate Lewis number $\mathrm{Le}_{\mathrm{Z}}=\frac{1}{4}, \frac{1}{3}, \frac{1}{2}, \frac{3}{4}, 1, \frac{3}{2}$ and 2 ; the dotted curve represents $S / S_{\mathrm{m}}=\left(Q^{2 / 3}-1\right) /(Q-1)$. The thin crosses mark solutions that are plotted in more detail in figure 5.

to $S_{\mathrm{m}}$, varies between about $\frac{1}{2}$ and $\frac{2}{3}$ for any values of $Q$ and $\mathrm{Le}_{\mathrm{Z}}$ in the ranges $1<Q \leq 5$ and $\frac{1}{4} \leq \mathrm{Le}_{\mathrm{Z}} \leq 2$.

Of course, the values of $a_{\mathrm{m}}\left(Q, \mathrm{Le}_{\mathrm{Z}}\right)$ and $S_{\mathrm{m}}\left(Q, \mathrm{Le}_{\mathrm{Z}}\right)$ do vary considerably with $Q$ and $\mathrm{Le}_{\mathrm{Z}}$. Both of these variations are illustrated in figure 4, in which the dominant changes with $Q$, both as $Q \rightarrow 1$ and for moderately large values of $Q$, appear to be normalized out of the figures by plotting $S_{\mathrm{m}} \times Q^{1 / 2} /(Q-1)$ and $a_{\mathrm{m}} \times Q^{3 / 2} /(Q-1)^{3}$ as functions of $Q$ for various values of $\mathrm{Le}_{\mathrm{Z}}$. For fixed values of $Q$ greater than about 2 and for values of $\mathrm{Le}_{\mathrm{Z}}$ greater than about $\frac{1}{4}$, the lower curves in figure 4 also show that $S_{\mathrm{m}}$ varies in approximate proportion to $\mathrm{Le}_{\mathrm{Z}}^{-2 / 3}$ and $a_{\mathrm{m}}$ is appoximately inversely proportional to $\mathrm{Le}_{\mathrm{Z}}$. On the other hand, when approaching the flammability limit $Q \rightarrow 1$, all dependence on $\mathrm{Le}_{\mathrm{Z}}$ disappears, as seen in the upper figures.

The structure of the flame varies with the heat of reaction $Q$ and intermediate Lewis number $\mathrm{Le}_{\mathrm{Z}}$ as well as location in the parameter-space of solution curves (seen in figure 3 ). Figure 5 shows a number of profiles of the solutions $F, Z$ and $T$ as functions of $S x$, so that most of the flame structure can be shown on the same scale in all cases. The fuel Lewis number $\mathrm{Le}_{\mathrm{F}}$ is set to one in all of these profiles; changing its value would scale the curves for $F$ horizontally and the curves for $Z$ vertically, as seen in the solutions (36). A notable feature of these solutions is that the relative magnitude and extent of the profiles of intermediate concentration $Z$ are reduced in cases where the maximum temperature $T$ is not much above one, as found when $Q$ 

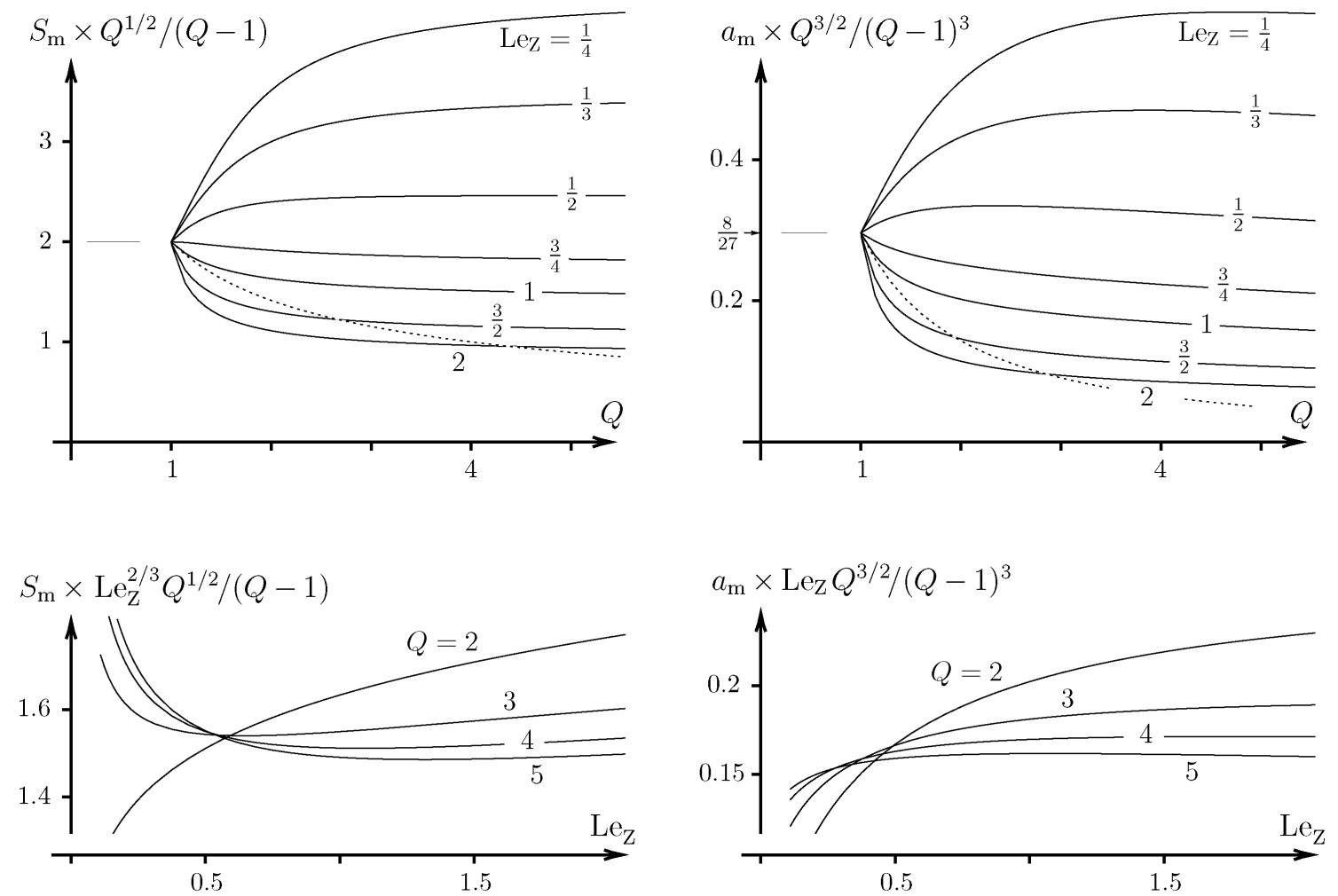

Figure 4. Dependence of the maximum flame speed $S_{\mathrm{m}}$ and the maximum heat-loss parameter $a_{\mathrm{m}}$ on the heat of reaction $Q$ and the intermediate Lewis number $\mathrm{Le}_{\mathrm{Z}}$. The dotted paths in the upper graphs are plotted using the formulae (53).
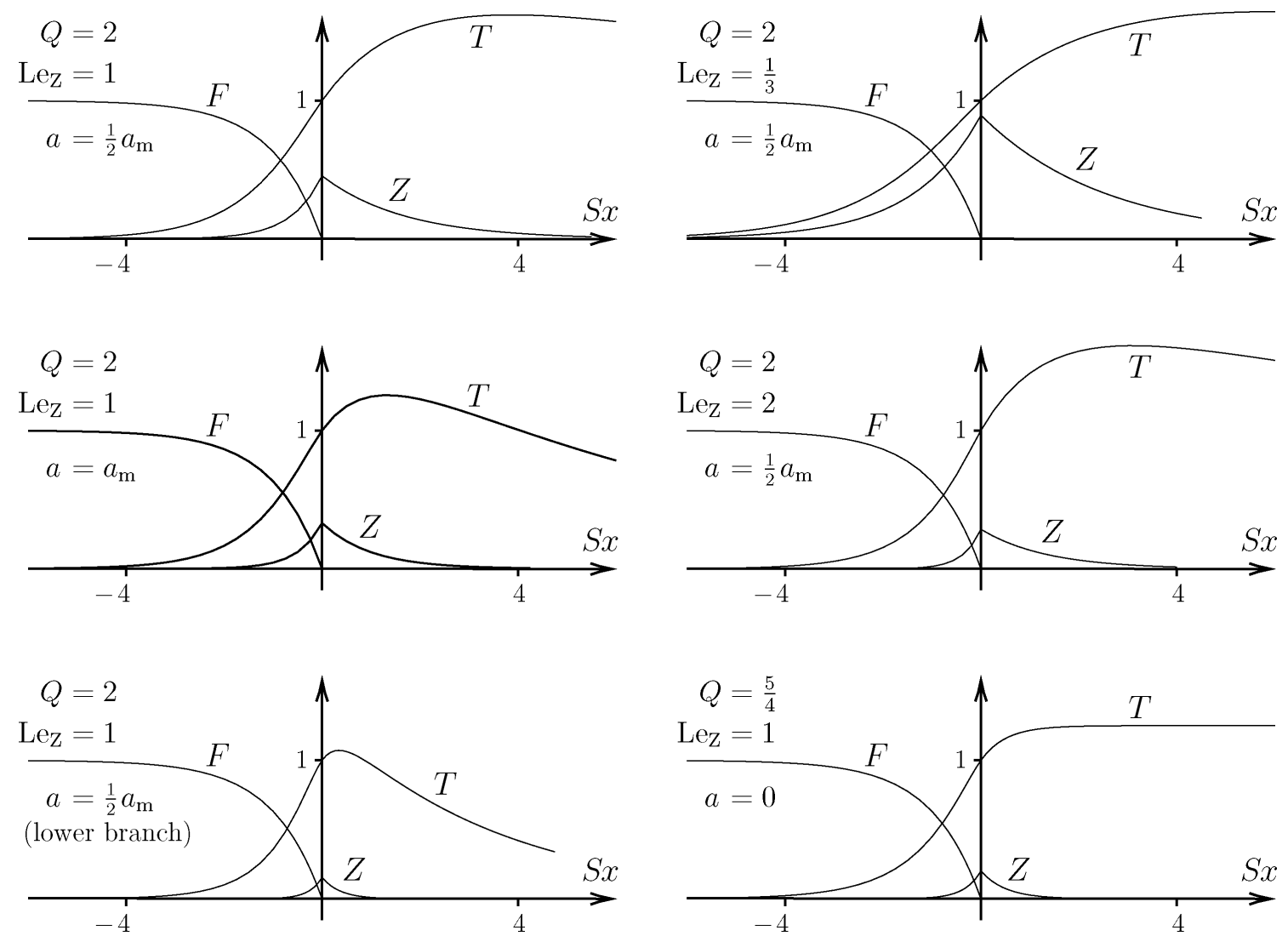

Figure 5. Variations of $F, Z$ and $T$ with position $x$ (scaled using the flame-speed $S$ ). Only the lower left graph is plotted using a value of $S$ that is on the lower branch of solution. The curves for $F$ and $Z$ are all calculated for $\operatorname{Le}_{\mathrm{F}}=1$. 
is decreased or when solutions move towards (or onto) the lower branch of solutions. In these cases the flame speed $S$ also decreases, as can be noted from figures 3 and 4 .

\subsection{Linear stability of a steadily propagating planar flame}

In considering a small sinusoidal oscillatory disturbance of wavenumber $k$ to the steady solutions just described, the branching-chemistry interface can be taken to follow the path $x=\mathrm{e}^{\lambda t+i k y}$, with the magnitude of $\left|\mathrm{e}^{\lambda t+i k y}\right|$ assumed to be very small. The disturbance increases with time if the real part of the 'growth-rate' $\lambda$ is positive. Disturbances in $F, Z$ and $T$ should also be proportional to $\mathrm{e}^{\lambda t+i k y}$ and can be taken to have the form

$$
F \sim F_{0}+\mathrm{e}^{\lambda t+i k y} F_{1}(x), \quad Z \sim Z_{0}+\mathrm{e}^{\lambda t+i k y} Z_{1}(x), \quad \frac{T-T_{\mathrm{c}}}{T-T_{\mathrm{c}}} \sim T_{0}+\mathrm{e}^{\lambda t+i k y} T_{1}(x)
$$

with errors being of order $\left|\mathrm{e}^{\lambda t+i k y}\right|^{2} \ll 1$. Substituting this into the model equations (33) leads to the differential equations that must be satisfied by $F_{1}, Z_{1}$ and $T_{1}$

$$
\begin{aligned}
F_{1}^{\prime \prime}-\mathrm{Le}_{\mathrm{F}} S F_{1}^{\prime}-\left(\mathrm{Le}_{\mathrm{F}} \lambda+k^{2}\right) F_{1} & =0 \\
Z_{1}^{\prime \prime}-\mathrm{Le}_{\mathrm{Z}} S Z_{1}^{\prime}-\left(\mathrm{Le}_{\mathrm{Z}} \lambda+k^{2}+1\right) Z_{1} & =0 \\
T_{1}^{\prime \prime}-S T_{1}^{\prime}-\left(\lambda+k^{2}+a\right) T_{1} & =-Q Z_{1} / \mathrm{Le}_{\mathrm{F}}
\end{aligned}
$$

in which we can take $F_{1} \equiv 0$ for $x>0$. Because instabilities must arise from the flame itself the disturbances should all decay to zero at infinity. That is

$$
\lim _{x \rightarrow-\infty}\left(F_{1}, Z_{1}, T_{1}\right)=(0,0,0), \quad \lim _{x \rightarrow \infty}\left(Z_{1}, T_{1}\right)=(0,0)
$$

Using Taylor expansions of the expressions (41) to apply the jump conditions (32) at the interface $x=\mathrm{e}^{\lambda t+i k y}$ leads to the form of the jump conditions

$\left[F_{0}^{\prime \prime}+F_{1}^{\prime}\right]+\left[Z_{0}^{\prime \prime}+Z_{1}^{\prime}\right]=\left[T_{0}^{\prime \prime}+T_{1}^{\prime}\right]=\left[F_{0}^{\prime}+F_{1}\right]=\left[Z_{0}^{\prime}+Z_{1}\right]=\left[T_{0}^{\prime}+T_{1}\right]=T_{0}^{\prime}+T_{1}=0$

which can be applied at $x=0^{ \pm}$. This does not include the condition $F T_{n}=0$, at $x=0$, which is already satisfied since $F_{0} \equiv F_{1} \equiv 0$ for $x>0$. In these jump conditions, we can make use of the relations $\left[F_{0}^{\prime}\right]=\operatorname{Le}_{\mathrm{F}} S,\left[F_{0}^{\prime \prime}\right]=\operatorname{Le}_{\mathrm{F}}^{2} S^{2},\left[Z_{0}^{\prime}\right]=-\operatorname{Le}_{\mathrm{F}} S,\left[Z_{0}^{\prime \prime}\right]=-\mathrm{Le}_{\mathrm{F}} \mathrm{Le}_{\mathrm{Z}} S^{2}$ and $\left[T_{0}^{\prime}\right]=\left[T_{0}^{\prime \prime}\right]=0$. Thus, the conditions can be written as

$$
\begin{aligned}
{\left[F_{1}^{\prime}\right]+\left[Z_{1}^{\prime}\right] } & =\left(\mathrm{Le}_{\mathrm{Z}}-\mathrm{Le}_{\mathrm{F}}\right) \mathrm{Le}_{\mathrm{F}} S^{2}, \quad\left[T_{1}^{\prime}\right]=\left[T_{1}\right]=0 \\
{\left[F_{1}\right] } & =-\mathrm{Le}_{\mathrm{F}} S, \quad\left[Z_{1}\right]=\mathrm{Le}_{\mathrm{F}} S \quad \text { and } \quad T_{0}^{\prime}+T_{1}=0
\end{aligned}
$$

with $T_{0}^{\prime}$, at $x=0$, already known from (39), with $\bar{F}=0$.

Solutions are

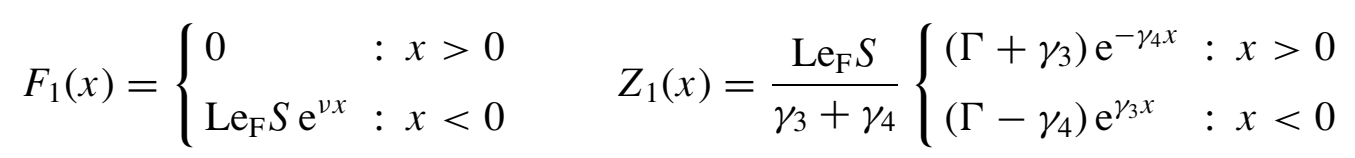

and

$$
T_{1}(x)=\frac{Q S}{\mu_{3}+\mu_{4}} \begin{cases}\frac{\Gamma+\mu_{4}+\gamma_{3}-\gamma_{4}}{\left(\gamma_{4}-\mu_{4}\right)\left(\gamma_{3}+\mu_{4}\right)} \mathrm{e}^{-\mu_{4} x} & : x>0 \\ \frac{\Gamma-\mu_{3}+\gamma_{3}-\gamma_{4}}{\left(\gamma_{4}+\mu_{3}\right)\left(\gamma_{3}-\mu_{3}\right)} \mathrm{e}^{\mu_{3} x} & : x<0\end{cases}
$$




$$
+\frac{Q S}{\gamma_{3}+\gamma_{4}} \begin{cases}\frac{\Gamma+\gamma_{3}}{\left(\mu_{3}+\gamma_{4}\right)\left(\mu_{4}-\gamma_{4}\right)} \mathrm{e}^{-\gamma_{4} x} & : x>0 \\ \frac{\Gamma-\gamma_{4}}{\left(\mu_{3}-\gamma_{3}\right)\left(\mu_{4}+\gamma_{3}\right)} \mathrm{e}^{\gamma_{3} x} & : x<0\end{cases}
$$

in which all conditions, apart from the final one $\left(T_{0}^{\prime}+T_{1}=0\right)$ are satisfied. Applying this last condition leads to the algebraic equation, or dispersion relation

$$
\frac{\Gamma-\gamma_{4}}{\left(\gamma_{3}+\gamma_{4}\right)\left(\gamma_{3}+\mu_{4}\right)}-\frac{\Gamma-\mu_{3}+\gamma_{3}-\gamma_{4}}{\left(\mu_{3}+\mu_{4}\right)\left(\mu_{3}+\gamma_{4}\right)}=\frac{\left(\gamma_{3}-\mu_{3}\right)\left(\gamma_{1} \mu_{1}-\gamma_{2} \mu_{2}\right)}{\left(\gamma_{1}+\gamma_{2}\right)\left(\mu_{1}+\mu_{2}\right)\left(\gamma_{1}+\mu_{2}\right)\left(\gamma_{2}+\mu_{1}\right)}
$$

which needs to be solved for the growth-rate $\lambda$ as a function of the wavenumber $k$ and all other parameters $\left(Q, S, \mathrm{Le}_{\mathrm{F}}, \mathrm{Le}_{\mathrm{Z}}\right.$ and $\left.a\right)$ subject to the relation (40). The new constants $\Gamma, v, \gamma_{3}, \gamma_{4}$, $\mu_{3}$ and $\mu_{4}$ appearing in these solutions are defined as

$$
\begin{array}{ll}
\Gamma=\left(\operatorname{Le}_{\mathrm{F}}-\mathrm{Le}_{\mathrm{Z}}\right) S-v & \gamma_{\{3,4\}}=\frac{1}{2}\left(\sqrt{4\left(\operatorname{Le}_{\mathrm{Z}} \lambda+k^{2}+1\right)+\operatorname{Le}_{\mathrm{Z}}^{2} S^{2}} \pm \mathrm{Le}_{\mathrm{Z}} S\right) \\
v=\frac{1}{2}\left(\sqrt{4\left(\operatorname{Le}_{\mathrm{F}} \lambda+k^{2}\right)+\mathrm{Le}_{\mathrm{F}}^{2} S^{2}}+\mathrm{Le}_{\mathrm{F}} S\right) & \mu_{\{3,4\}}=\frac{1}{2}\left(\sqrt{4\left(\lambda+k^{2}+a\right)+S^{2}} \pm S\right) .
\end{array}
$$

Unlike the flame-speed $S$ it can be seen that linear stability should depend on the Lewis number of the fuel $\mathrm{Le}_{\mathrm{F}}$ as well as all other parameters. Given a steady flame solution with parameters satisfying the relation (40) and any chosen value of $\mathrm{Le}_{\mathrm{F}}$, the dispersion relation (42) can be solved numerically to find the dependence of the least stable growth-rate $\lambda$ on the wavenumber $k$.

For values of $\mathrm{Le}_{\mathrm{F}}$ below unity the least stable root is real. This root is always zero at $k=0$ but elsewhere it varies with $k$. For non-zero wavenumbers it first becomes positive as $\mathrm{Le}_{\mathrm{F}}$ decreases through a threshold value, as shown in figure 6 for the adiabatic case $a=0$. The maximum value of this threshold lies at $k=0$ so that, if $\operatorname{Le}_{\mathrm{F}}$ is decreased by a small amount from its maximum threshold value, marginal instability first appears at very small wavenumbers, generating a well-known 'cellular' flame structure [5].

For small enough heats of reaction $Q$ the real part of two complex conjugate roots becomes positive as $\mathrm{Le}_{\mathrm{F}}$ increases through another threshold value, generating an oscillatory instability. As seen in figure 6 for the adiabatic case $a=0$, this happens at values of $\mathrm{Le}_{\mathrm{F}}$ that are greater than unity. The minimum value of $\mathrm{Le}_{\mathrm{F}}$, at which marginal instability first appears, lies at a non-zero wavenumber $k$. Figure 7 shows how the value of $k$ for marginal oscillatory instability varies with $\mathrm{Le}_{\mathrm{Z}}$ and $Q$ for adiabatic flames, broadly scaling with $Q-1$ over a range of values of $\mathrm{Le}_{\mathrm{Z}}$, as $Q$ varies.

It can be noted that the dependence of all stability thresholds on the intermediate Lewis number $\mathrm{Le}_{\mathrm{Z}}$ disappears as $Q-1$ becomes very small. At this stage, there appears to be an almost direct linear relationship between the marginally unstable values of $\operatorname{Le}_{\mathrm{F}}-1$ and $Q-1$.

The marginal values of $\mathrm{Le}_{\mathrm{F}}$ for the oscillatory instability grow very rapidly as $Q$ increases. The upper right graph in figure 6 , which plots the variation of $1 / \mathrm{Le}_{\mathrm{F}}$ with $Q$, strongly suggests that the marginal values of $\mathrm{Le}_{\mathrm{F}}$ become infinite at a finite and still relatively small heat of reaction (around $Q=1.3$ to 1.4 for reasonable, order one values of $L_{\mathrm{Z}}$ ) at least for adiabatic flames. The dotted lines in the graph, which extrapolate the calculated solutions to $\mathrm{Le}_{\mathrm{F}}=\infty$, indicate that the value of $\mathrm{Le}_{\mathrm{F}}$ grows in proportion to $\left(Q_{\mathrm{c}}-Q\right)^{-1}$ as $Q$ approaches the critical value $Q_{\mathrm{c}}\left(\mathrm{Le}_{\mathrm{Z}}\right)$ where the marginal value of $\mathrm{Le}_{\mathrm{F}}$ becomes infinite. The instability is thus seen to disappear for all but relatively small values of $Q$ and, even then, the fuel Lewis number $\mathrm{Le}_{\mathrm{F}}$ that is required for instability tends to be rather large.

The marginal values of $\mathrm{Le}_{\mathrm{F}}$ for the cellular instability all decrease as $Q$ increases at any fixed value of $\mathrm{Le}_{\mathrm{Z}}$. The exaggerated variation of $\mathrm{Le}_{\mathrm{Z}}$ in figure 6 between $2^{-7}$ and $2^{3}$ is used 

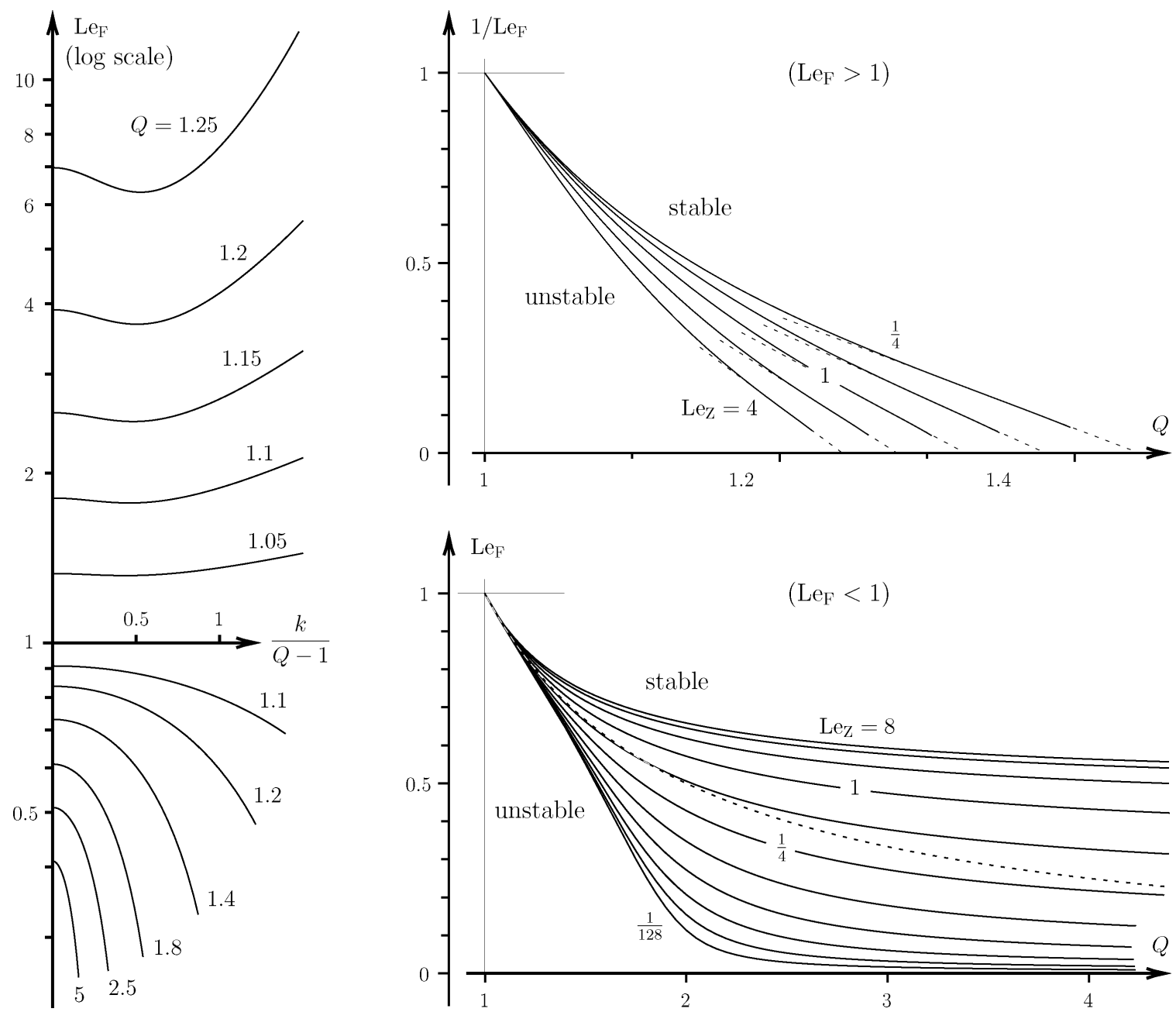

Figure 6. Neutral stability curves under adiabatic conditions (having $a=0$ ). The left graph shows upper and lower instability-threshold values of the fuel Lewis number $\mathrm{Le}_{\mathrm{F}}$, as a function of wavenumber $k$, for different values of the heat of reaction $Q$ with the intermediate Lewis number fixed at $\mathrm{Le}_{\mathrm{Z}}=1$; above the upper threshold an oscillatory instability arises and below the lower threshold there is a cellular instability. The right hand graphs show how the values of $\mathrm{Le}_{\mathrm{F}}$ at marginal instability (or $1 / \mathrm{Le}_{\mathrm{F}}$ in the upper figure) vary with $Q$ over a range of values of $\mathrm{Le}_{\mathrm{Z}}$ (varying in powers of 2). The dotted lines extrapolate $\mathrm{Le}_{\mathrm{F}}$ to infinity in the upper graph and trace $\mathrm{Le}_{\mathrm{F}}=1 / Q$ in the lower graph.

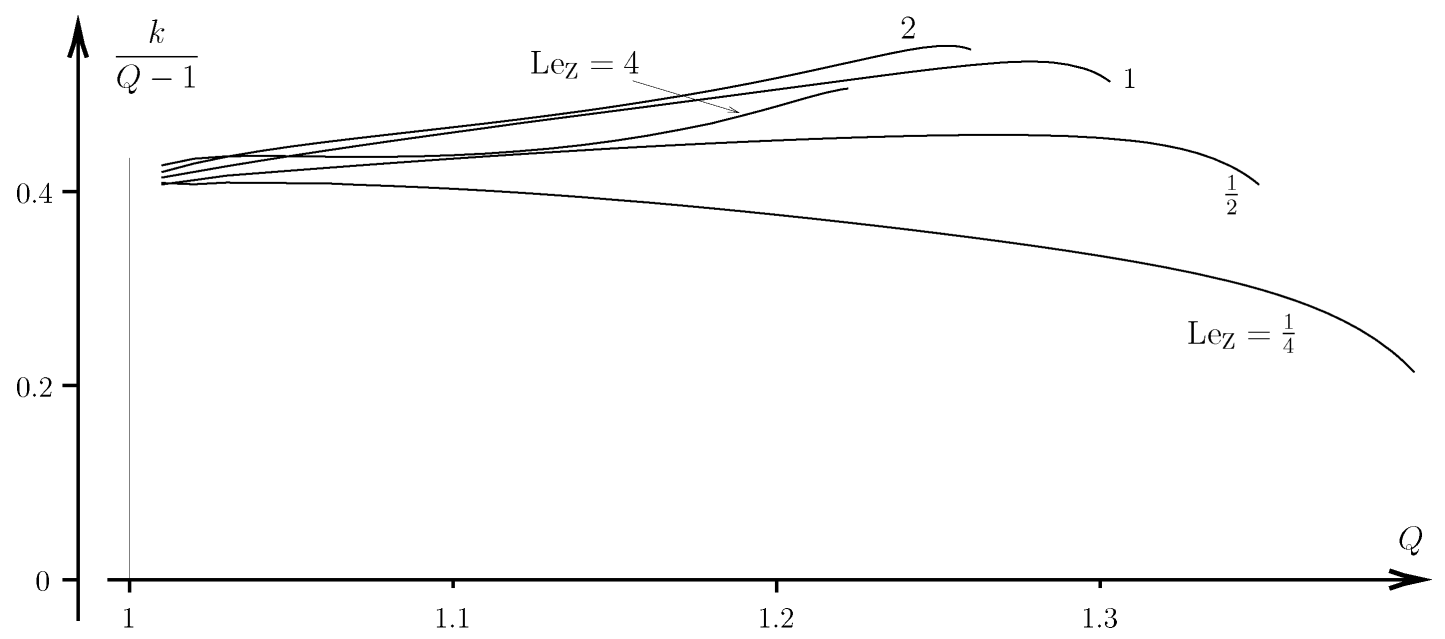

Figure 7. Dependence of the wavenumber of marginal oscillatory instability on heat of reaction $Q$ at fixed values of $\mathrm{Le}_{\mathrm{Z}}$ for adiabatic flames. 

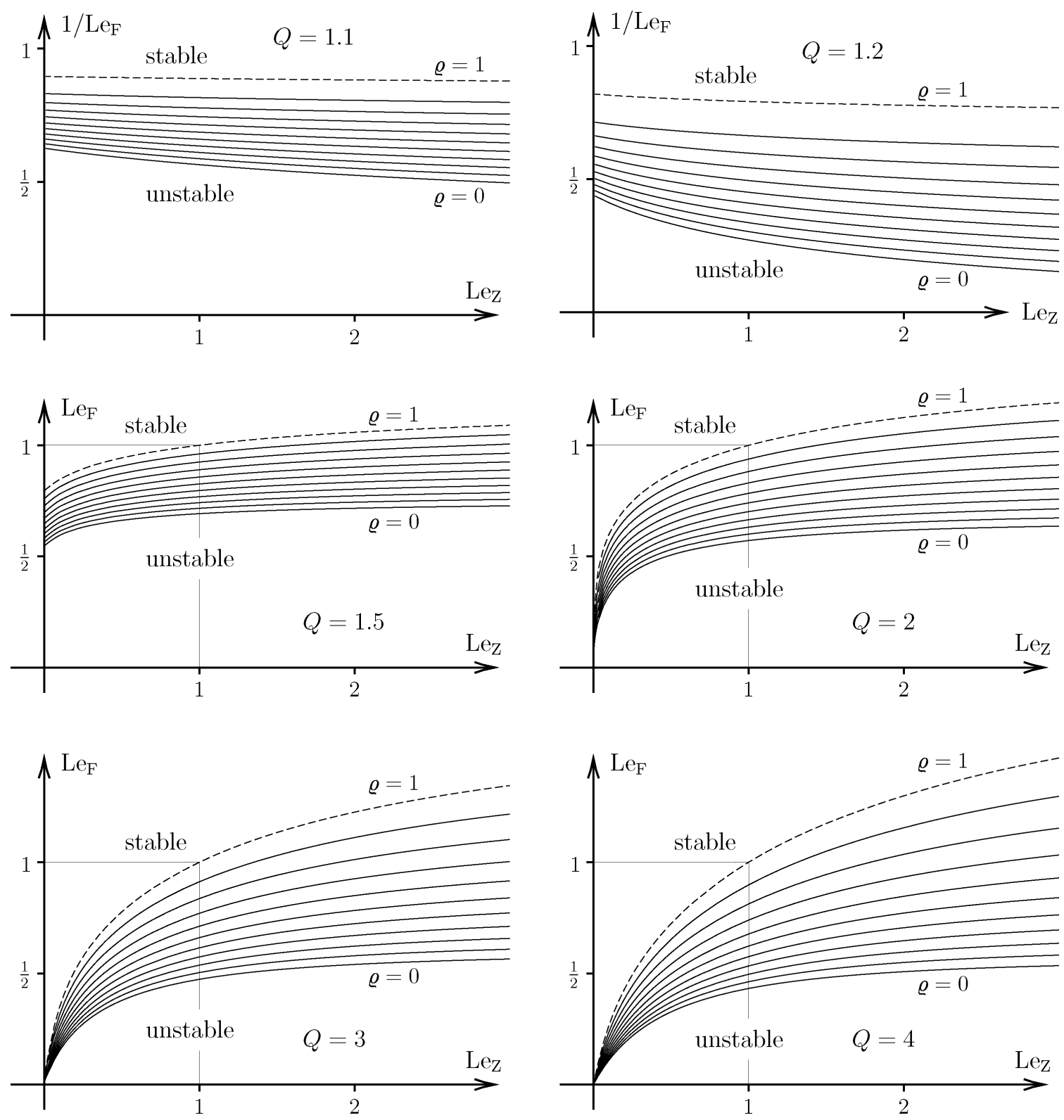

Figure 8. Variation of marginal stability boundaries due to heat loss, in the space of the Lewis numbers $\mathrm{Le}_{\mathrm{F}}$ and $\mathrm{Le}_{\mathrm{Z}}$. The parameter $\varrho$ measures the reduction in propagation speed from its adiabatic maximum $(\varrho=0)$ to the turning point $(\varrho=1)$. Curves are plotted at fixed values of $\varrho$, changing in steps of 0.1 .

to demonstrate that the dependence of this instability on $\mathrm{Le}_{\mathrm{Z}}$ is rather weak. The dotted curve plots the function $\mathrm{Le}_{\mathrm{F}}=1 / Q$ which is an estimate of the marginal value of $\mathrm{Le}_{\mathrm{F}}$ that is obtained later (in Section 5.2) for the limit in which $Q-1 \ll 1$. This curve follows the adiabatic stability boundary for intermediate Lewis number $\mathrm{Le}_{\mathrm{Z}}=\frac{1}{2}$ quite well for $Q$ below about 2.5, and it offers a rough overall approximation for order one values of $\mathrm{Le}_{\mathrm{Z}}$ and $Q$.

Heat losses tend to be destabilizing, as illustrated in figure 8 for selected values of $Q$. At $Q=1.1$ and 1.2 the upper two graphs show how the marginal values of $\mathrm{Le}_{\mathrm{F}}$ for the oscillatory instability decrease as the effect of heat loss is increased. The parameter used to measure the effect of heat loss in figure 8 is $\varrho$ defined as

$$
\varrho=\frac{S_{\mathrm{m}}-S}{S_{\mathrm{m}}-S\left(a_{\mathrm{m}}\right)}
$$

which takes the value zero in the adiabatic case ( $a=0$ with $\left.S=S_{\mathrm{m}}\right)$ and one at the turning point where $a=a_{\mathrm{m}}$ and $S=S\left(a_{\mathrm{m}}\right)$. Values of $\varrho$ greater than unity correspond to solutions on 
the lower branch of solution; such solutions are always unstable. For $Q=1.5,2,3$ and 4, the lower four graphs in figure 8 show the effect of increasing heat loss on the marginal stability boundaries for the cellular instability. The effect is always to reduce the range of parameters in which stability is found.

Another feature that can be noted from both figure 6 and figure 8 is that reducing the intermediate Lewis number $\mathrm{Le}_{\mathrm{Z}}$ tends towards stabilizing the cellular instability but increasing the chance of generating the oscillatory instability (particlularly for larger values of $\mathrm{Le}_{\mathrm{F}}$ ). When the heat of reaction $Q$ is very close to unity changing Le $_{Z}$ has very little effect. Cases for which $Q-1 \ll 1$ are examined in the next section.

\section{Flames with low concentrations of intermediates}

When values of $Z$ and $S$ are relatively small it can be seen from the solutions (36) and (37) that $T$ and $F$ tend to vary on length scales, $x=\mathrm{O}\left(S^{-1}\right)$, that are large compared with the scale, $x=\mathrm{O}(1)$, on which $Z$ changes. This leads to a second asymptotic limit in which $F, Z$ and $T$ can be characterized in the following way

$$
F \sim \bar{F}+\epsilon f(n), \quad Z \sim \epsilon \zeta(n), \quad \frac{T-T_{\mathrm{c}}}{1-T_{\mathrm{c}}} \sim 1+\epsilon S(n)
$$

for $\epsilon \ll 1$, where $n$ is a coordinate measuring distance normal to the surface $\mathbf{x}=\mathbf{R}$ where the branching reaction is taken to occur. If the value of $\epsilon$ is chosen to be the actual value of $Z$ at the branching reaction-sheet, then the value of $\zeta$ is unity at $n=0$. At large values of $n$, the intermediate concentration $Z$ approaches zero, as seen in figure 5, and $F$ and $T$ vary relatively slowly, on the time-scale $\epsilon^{2} t$ and length-scale $\epsilon \mathbf{x}$. Correspondingly, the flame speed scales as $S=\mathrm{O}(\epsilon)$, so that the results shown in figure 4 indicate that $\ell=\mathrm{O}\left(\epsilon^{3}\right)$ for solutions to exist.

When $n$ is of order one, an inner asymptotic problem is described, to leading order as $\epsilon \rightarrow 0$, by the equations

$$
f_{n n}=s_{n n}+\frac{Q}{\operatorname{Le}_{\mathrm{F}}} \zeta=\zeta_{n n}-\zeta=0
$$

and jump conditions at $n=0$

$$
\left[f_{n}\right]+\left[\zeta_{n}\right]=\left[s_{n}\right]=[f]=[\zeta]=[s]=s=\bar{F} s_{n}=0 .
$$

Using the condition that $\zeta(0)=1$ the solution is then

$$
f=f_{1} n+|n|, \quad \zeta=\mathrm{e}^{-|n|}, \quad s=s_{1} n+\frac{Q}{\operatorname{Le}_{\mathrm{F}}}\left(1-\mathrm{e}^{-|n|}-|n|\right) .
$$

If $\bar{F}=0$ and if $n$ points into the direction where the gas is unburnt (or $T<1$ ), then $f_{1}=1$ so that $f \equiv 0$ where $T>1$; if $n$ points in the opposite direction then $f_{1}=-1$. The condition $\bar{F} s_{n}=0$ requires that $\bar{F} s_{1}=0$ since $s_{n}=s_{1}$ at $n=0$.

Viewed from the far field the exponentials in this result are lost, leaving only straight line asymptotic behaviour. Thus, over the range $1 \ll|n| \ll \epsilon^{-1}$, the solutions can be written as

$$
F \sim \bar{F}+f_{1} \epsilon n+|\epsilon n| \quad \frac{T-T_{\mathrm{c}}}{1-T_{\mathrm{c}}} \sim 1+\epsilon \frac{Q}{\mathrm{Le}_{\mathrm{F}}}+\epsilon s_{1} n-\frac{Q}{\mathrm{Le}_{\mathrm{F}}}|\epsilon n|
$$

which are accurate to order $\epsilon n$. Through eliminating $\epsilon$ it is now found that the outer asymptotic problem for $|n| \gg 1$ satisfies the jump conditions at $n=0$

$$
[F]=[T]=\left(T_{n}^{+}+T_{n}^{-}\right) F=0, \quad \frac{1-T_{\mathrm{c}}}{\mathrm{Le}_{\mathrm{F}}} Q\left[F_{n}\right]=-\left[T_{n}\right]=2(T-1)>0 .
$$


It can be noted that the asymptotic value of $T$ as $\mathbf{x} \rightarrow \mathbf{R}$ is not the same here as the value of $T$ at $\mathbf{x}=\mathbf{R}$ in the inner solution that takes into account the intermediate reactant $Z$. Rather, these jump conditions are to be used in a model where both the chain-branching and recombination reactions occur in a thin interface, at $n=0$ or $\mathbf{x}=\mathbf{R}$. The appropriate version of equations (31) that $F$ and $T$ should satisfy is then

$$
\begin{aligned}
\operatorname{Le}_{\mathrm{F}}\left(F_{t}+\mathbf{u} \cdot \nabla F\right) & =\nabla^{2} F \\
T_{t}+\mathbf{u} \cdot \nabla T & =\nabla^{2} T-\ell
\end{aligned}
$$

with the jump conditions (47) applied at a sheet where all chemistry is taken to occur.

In these equations, the intermediate concentration $Z$ plays no further role and the asymptotic value of the temperature $T$, extrapolated to the interface, is no longer equal to unity. In fact this further development of the model is asymptotically justified only if $T>1$ and $T-1 \ll 1$ at $n=0$. As a model, however, it could still be applied for any positive values of $T-1$ at the interface.

Using (28) and (30) second-order effects for $\theta \gg 1$ can still be accommodated (provided $\left.\epsilon \gg \theta^{-1}\right)$ by replacing the factor $2(T-1)$ in $(47)$ by $2\left(T-T_{\mathrm{s}}^{\prime}\right)$ where

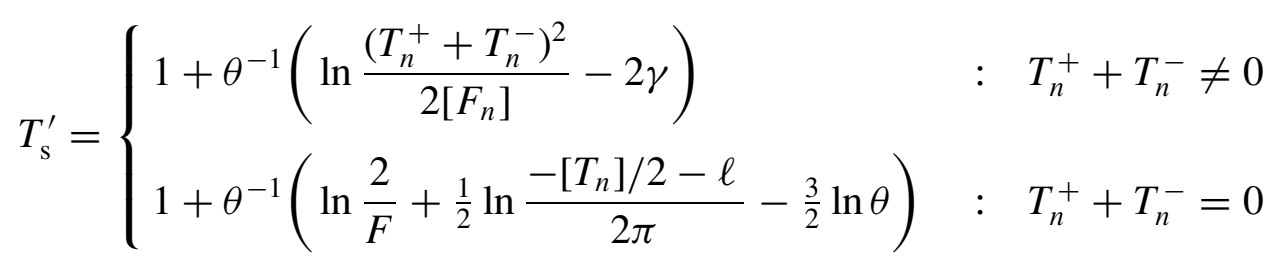

since $Z \sim \epsilon \sim\left[F_{n}\right] / 2$ and $\left(1-T_{\mathrm{c}}\right) Q\left[F_{n}\right] / \mathrm{Le}_{\mathrm{F}} \sim-\left[T_{n}\right]$ at the interface. These formulae apply if $T_{\mathrm{r}}$ is used as the reference temperature $\left(T_{\mathrm{s}}=T_{\mathrm{r}}\right)$ in the dimensionless scalings (21) and (22). If the dimensional reference temperature $T_{\mathrm{s}}$ is defined to be the asymptotic temperature of the branching reaction sheet itself then conditions (47) are already accurate to second order and the formula (49) can be used to identify the value of $T_{\mathrm{s}} \sim T_{\mathrm{s}}^{\prime} T_{\mathrm{r}}$ in cases where $\left[F_{n}\right] \ll 1$ and equations (48) are used to solve for the flame structure.

\subsection{Steady solutions}

With velocity $\mathbf{u}$ having speed $S$ in the $x$ direction, steady solutions of (48) in the form of (34), with linear rate of heat-loss $\ell=a\left(T-T_{\mathrm{c}}\right)$ as before, satisfy

$$
\mathrm{Le}_{\mathrm{F}} S F_{0}^{\prime}=F_{0}^{\prime \prime}, \quad S T_{0}^{\prime}=T_{0}^{\prime \prime}-a T_{0}
$$

subject to boundary conditions $F_{0}(-\infty)=1$ with $T_{0}(-\infty)=F_{0}(\infty)=T_{0}^{\prime}(\infty)=0$ and the jump conditions at an interface, which can be placed at $x=0$ without loss of generality

$$
\left[T_{0}\right]=F_{0}\left(0^{ \pm}\right)=0, \quad \frac{Q}{\mathrm{Le}_{\mathrm{F}}}\left[F_{0}^{\prime}\right]=-\left[T_{0}^{\prime}\right]=2\left(T_{0}-1\right) .
$$

These conditions anticipate that $T_{0}^{\prime}\left(0^{+}\right)+T_{0}^{\prime}\left(0^{-}\right) \neq 0$ so that $F_{0}$ should be zero at the interface, $x=0$. Solutions satisfying all but the last two conditions are

$$
F_{0}(x)=\left\{\begin{array}{ll}
0 & : x>0 \\
1-\mathrm{e}^{\mathrm{Le}_{\mathrm{F}} S x} & : x<0
\end{array} \quad T_{0}(x)=\bar{T} \begin{cases}\mathrm{e}^{-\mu_{2} x} & : x>0 \\
\mathrm{e}^{\mu_{1} x} & : x<0\end{cases}\right.
$$

with $\mu_{1}$ and $\mu_{2}$ as defined in (38). The remaining conditions at $x=0$ now give

$$
\bar{T}=1+\frac{1}{2} Q S \quad \text { and } \quad a=\frac{Q^{2} S^{2}}{(2+Q S)^{2}}-\frac{1}{4} S^{2} .
$$


As before it can be noted that the propagation speed $S$ is independent of the fuel Lewis number $\mathrm{Le}_{\mathrm{F}}$. In this limit, where the role of the intermediate $Z$ has been eliminated, it is also independent of $\mathrm{Le}_{\mathrm{Z}}$.

The formula (52) can be manipulated to show that maximum values of $S$ and $a$ are

$$
S_{\mathrm{m}}=2(Q-1) / Q, \quad a_{\mathrm{m}}=\left(Q^{2 / 3}-1\right)^{3} / Q^{2} .
$$

At the turning point where $a=a_{\mathrm{m}}$ the value of $S$ is $S=2\left(Q^{2 / 3}-1\right) / Q$, which shows that $S / S_{\mathrm{m}}=\left(Q^{2 / 3}-1\right) /(Q-1)$. This function is plotted as the dotted curve in the lower graph in figure 3. Points that are plotted for the heat of reaction $Q=1$ in figures 3 and 4 are in fact determined by the limits of the formulae above as $Q \rightarrow 1$. The dotted curves in the upper graphs of figure 4 are plotted using the formulae (53), from which it can be seen that the values predicted become less reliable as $Q-1$ increases, as might be expected.

The behaviour of solutions when $Q-1$ is small can be seen more clearly through rewriting the formula for $a$ as

$$
\begin{aligned}
\frac{a Q^{2}}{(Q-1)^{3}} & =\frac{\left(S / S_{\mathrm{m}}\right)^{2}\left(1+Q-2 S / S_{\mathrm{m}}-(Q-1)\left(S / S_{\mathrm{m}}\right)^{2}\right)}{\left(1+(Q-1) S / S_{\mathrm{m}}\right)^{2}} \\
& =2\left(S / S_{\mathrm{m}}\right)^{2}\left(1-S / S_{\mathrm{m}}\right)+\mathrm{O}(Q-1) .
\end{aligned}
$$

Thus $a$ varies in proportion to $S^{2}\left(S_{\mathrm{m}}-S\right)$ and is of order $(Q-1)^{3}$ in magnitude, when $Q$ is close to unity. Corresponding curves relating $S$ and $a$ are plotted as dotted paths in the upper graphs in figure 3 .

Two solution profiles based on (51) are shown in figure 9 for the Lewis number $\mathrm{Le}_{\mathrm{F}}=1$. The left graph is an adiabatic solution and the right graph is a solution at maximum heat-loss, having $a=a_{\mathrm{m}}$. The dotted curves present the temperature profiles from the fuller model (37) calculated by taking $\mathrm{Le}_{\mathrm{Z}}=1$. In both of these solutions the temperature extrapolated to the flame sheet (at $x=0$ ) is not very much greater than $T=1$ so that the assumptions leading to the solutions (51) should be satisfied. This is confirmed by the close correspondence between the solid and dotted curves for temperature away from the origin. The solutions for $F$ are identical.

\subsection{Linear stability}

If the interface is at $x=\mathrm{e}^{\lambda t+i k y}$ and the steady solutions for $F$ and $T$ are disturbed in the manner shown in (41), then the functions $F_{1}$ and $T_{1}$ satisfy

$$
\begin{array}{r}
F_{1}^{\prime \prime}-\mathrm{Le}_{\mathrm{F}} S F_{1}^{\prime}-\left(\mathrm{Le}_{\mathrm{F}} \lambda+k^{2}\right) F_{1}=0 \\
T_{1}^{\prime \prime}-S T_{1}^{\prime}-\left(\lambda+k^{2}+a\right) T_{1}=0
\end{array}
$$
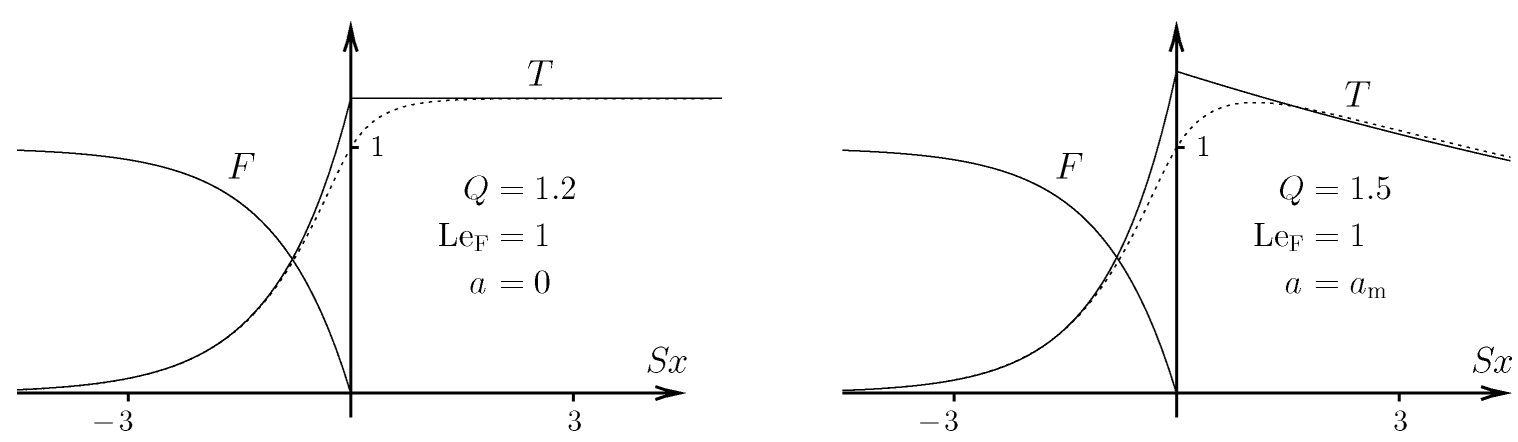

Figure 9. Variations of $F$ and $T$ with position $x$ (scaled using the flame-speed $S$ ) for an adiabatic solution (left) and for a solution at maximum heat-loss (right). Solid curves represent the solutions (51) and the dotted curves are the temperature according to the solution (37). 
and we can take $F_{1} \equiv 0$ for $x>0$. The jump conditions (47), applied to Taylor expansions of $F$ and $T$, can be rewritten as the conditions at $x=0^{ \pm}$

$$
F_{0}^{\prime}\left(0^{-}\right)+F_{1}\left(0^{-}\right)=\left[T_{0}^{\prime}+T_{1}\right]=0, \quad \frac{Q}{\mathrm{Le}_{\mathrm{F}}}\left[F_{0}^{\prime \prime}+F_{1}^{\prime}\right]=-\left[T_{0}^{\prime \prime}+T_{1}^{\prime}\right]=2\left(T_{0}^{\prime}+T_{1}\right) .
$$

From the solutions (51) with (52) and the formulae (38) or conditions (50) it can be shown that $F_{0}^{\prime}\left(0^{-}\right)=-\mathrm{Le}_{\mathrm{F}} S,\left[F_{0}^{\prime \prime}\right]=\mathrm{Le}_{\mathrm{F}}^{2} S^{2},\left[T_{0}^{\prime}\right]=-Q S$ and $\left[T_{0}^{\prime \prime}\right]=-Q S^{2}$, so that

$$
F_{1}\left(0^{-}\right)=\operatorname{Le}_{\mathrm{F}} S, \quad\left[T_{1}\right]=Q S, \quad \operatorname{Le}_{\mathrm{F}} Q S^{2}+\frac{Q}{\operatorname{Le}_{\mathrm{F}}}\left[F_{1}^{\prime}\right]=Q S^{2}-\left[T_{1}^{\prime}\right]=2\left(\mu_{1} \bar{T}+T_{1}^{-}\right)
$$

Finally, using the definitions (43), solutions that decay to zero as $x \rightarrow \pm \infty$ become

$F_{1}(x)=\left\{\begin{array}{ll}0 & : x>0 \\ \operatorname{Le}_{\mathrm{F}} S \mathrm{e}^{v x} & : x<0\end{array} T_{1}(x)=\frac{Q S}{\mu_{3}+\mu_{4}} \begin{cases}\left(\left(\mathrm{Le}_{\mathrm{F}}-1\right) S-v+\mu_{3}\right) \mathrm{e}^{-\mu_{4} x} & : x>0 \\ \left(\left(\mathrm{Le}_{\mathrm{F}}-1\right) S-v-\mu_{4}\right) \mathrm{e}^{\mu_{3} x} & : x<0\end{cases}\right.$

for values of $\lambda$ that satisfy the dispersion relation

$$
Q S\left(\operatorname{Le}_{\mathrm{F}} S-v\right)=2 \mu_{1}\left(1+\frac{1}{2} Q S\right)+2 Q S \frac{\left(\mathrm{Le}_{\mathrm{F}}-1\right) S-v-\mu_{4}}{\mu_{3}+\mu_{4}} .
$$

which, after using (52) and (53), can be written in the form

$$
\sqrt{\operatorname{Le}_{\mathrm{F}} \lambda+k^{2}+\mathrm{Le}_{\mathrm{F}}^{2} S^{2} / 4}+1=\frac{S_{\mathrm{m}}+\left(\mathrm{Le}_{\mathrm{F}}-1\right) S}{2}+\frac{\sqrt{\operatorname{Le}_{\mathrm{F}} \lambda+k^{2}+\mathrm{Le}_{\mathrm{F}}^{2} S^{2} / 4}-\left(\mathrm{Le}_{\mathrm{F}}-1\right) S / 2}{\sqrt{\lambda+k^{2}+S^{2} /\left(2-S_{\mathrm{m}}+S\right)^{2}}}
$$

with $0<S \leq S_{\mathrm{m}}=2(Q-1) / Q<2$ and $a$ replaced by $S^{2} /\left(2-S_{\mathrm{m}}+S\right)^{2}-\frac{1}{4} S^{2}$ after using (52) and (53). It can be noted that this equation is always satisfied if $\lambda=k=0$, so that $\lambda=0$ must be a solution whenever $k=0$. Also, in the case $\operatorname{Le}_{\mathrm{F}}=1$, it can be seen that $\lambda=-k^{2}$ is always a solution.

It is convenient to write $S=v S_{\mathrm{m}}$ and $S_{\mathrm{m}}=2 \delta$, so that the normalized flame speed $v$ and heat of reaction factor $\delta$ lie in the ranges $0<v \leq 1$ and $0<\delta=(Q-1) / Q<1$. It is also helpful to rescale the wavenumber and growth rate such that $k=\delta \mathrm{Le}_{\mathrm{F}} v \kappa$ and $\lambda=\delta^{2} \mathrm{Le}_{\mathrm{F}} v^{2} \sigma$. The dispersion relation can then be rewritten as

$$
\frac{1+\operatorname{Le}_{\mathrm{F}}\left(\sqrt{\sigma+\kappa^{2}+1}-1\right)}{\sqrt{\operatorname{Le}_{\mathrm{F}}\left(\sigma+\operatorname{Le}_{\mathrm{F}} \kappa^{2}\right)+1 /(1-\delta(1-v))^{2}}}=1-\delta(1-v)+\delta \operatorname{Le}_{\mathrm{F}} v\left(\sqrt{\sigma+\kappa^{2}+1}-1\right) .
$$

Defining a reduced fuel Lewis number as $l=\left(\operatorname{Le}_{\mathrm{F}}-1\right) / \delta$ and setting $\varpi=\sqrt{\sigma+\kappa^{2}+1}$, this equation takes the form

$$
2 v \varpi^{3}-(2+l) \varpi^{2}+2 l \varpi+l\left(\kappa^{2}-1\right)+2(1-v)=0
$$

to order $\delta$, when expanded about $\delta=0$ for order one values of $l$ and $\varpi$. Knowing that $\varpi=1$ when $\kappa=0$ leads to the factorization

$$
(\varpi-1-r)\left(2 v \varpi^{2}-(l+2-2 v-2 v r) \varpi+l-2+2 v+(4 v-l-2) r+2 v r^{2}\right)=0
$$

with $r$ satisfying

$$
r=-\frac{l \kappa^{2}+(6 v-l-2) r^{2}+2 v r^{3}}{6 v-4}
$$

Since $r$ can be taken equal to zero when $\kappa=0$, the latter equation can then be solved iteratively, so that the root $\varpi=1+r$ can be calculated for small values of $\kappa$. This leads to the formula 
for one possible value of $\sigma$, evaluated as $\sigma=\varpi^{2}-\left(1+\kappa^{2}\right)$

$$
\sigma_{1}=-\frac{l+3 v-2}{3 v-2} \kappa^{2}-2 \frac{3 v-l}{(6 v-4)^{3}} l^{2} \kappa^{4}+\mathrm{O}\left(\kappa^{6}\right) .
$$

The other two roots become

$$
\begin{aligned}
\sigma_{\{2,3\}} & =\frac{A}{8 v^{2}} \pm \frac{l+2(1-v)-2 v r}{8 v^{2}} \sqrt{B} \\
A & =(l-4 v+2)^{2}-4 v(7 v-4)-4 v^{2}\left(r(2+r)+2 \kappa^{2}\right) \\
B & =A-4 v(l-2)+4 v r(l+2)+8 v^{2}\left(\kappa^{2}-r(2+r)\right)
\end{aligned}
$$

although since $\varpi=\sqrt{\sigma+\kappa^{2}+1}$ represents the principal square root, which requires that $\operatorname{Re}(\varpi) \geq 0$, negative values of $\operatorname{Re}(\varpi)$ must be excluded. That is, if the expression

$$
\varpi=\frac{l+2(1-v)-2 v r \pm \sqrt{B}}{4 v}
$$

is found to produce a negative real part, then the root must be excluded.

The root $\sigma_{1}$ is positive over at least some small range of values of $\kappa \neq 0$ when the coefficient of $\kappa^{2}$ becomes positive in equation (56). That is, instability arises for some nonzero wavenumbers when $l<-(3 v-2)$, assuming that $v>\frac{2}{3}$. This describes a cellular instability that can form on the upper branch of solution at a low enough Lewis number of the fuel. On approaching the turning point from above, as $v \rightarrow \frac{2}{3}^{+}$, the instability grows extremely rapidly for any value of $l<0$. In the adiabatic case, for which $v=1$, the cellular instability begins as $l$ decreases through the maximum threshold value of $l=-1$.

At the turning point where $v=\frac{2}{3}$ it is also found, for $\kappa=0$, that the roots in equation (57) become precisely $\sigma=\frac{9}{32}\left(l+\frac{2}{3}\right)(l-2 \pm|l-2|)$, excluding the root that contains $-|l-2|$ when $l<\frac{2}{3}$. One root is always zero, while there is also a positive root for $l>2$. If $v$ is decreased below $\frac{2}{3}$ then the zero root becomes positive. Thus either complete linear instability (for $l>2$ ) or marginal instability (for $l \leq 2$ ) is found at the turning point, with disturbances of zero wavenumber, and all solutions are unstable below the turning point.

In the adiabatic case, $v=1$, equation (57) provides the roots

$$
\sigma= \begin{cases}\frac{1}{8}\left((l-2)^{2}-12 \pm i l \sqrt{l(8-l)}\right):|l-4| \leq 4 \\ \frac{1}{8}\left((l-2)^{2}-12 \pm l \sqrt{l(l-8)}\right):|l-4| \geq 4\end{cases}
$$

(still only considering $\kappa=0$ ). For negative values of $l$, the more positive root, arising from $-l \sqrt{l(l-8)}$, must be excluded. The other root is always negative. For positive values of $l$ it can be seen that the real part of two complex conjugate roots becomes positive when entering the range $l>2+2 \sqrt{3} \approx 5.464 \ldots$, representing a pulsating or oscillatory instability with zero wavenumber at a frequency of $(1+\sqrt{3}) \sqrt[4]{12} /(4 \pi) \approx 0.4046 \ldots$ As $l$ increases further the frequency decreases, reaching zero when the roots collide (and become real) at $l=8$. After this, at least one root remains positive for all larger values of $l$.

Still considering the adiabatic case $v=1$, with wavenumbers $\kappa \neq 0$, equation (55) shows that $r=-\frac{1}{2} l \kappa^{2}+\frac{1}{8}(l-4) l^{2} \kappa^{4}+\mathrm{O}\left(\kappa^{6}\right)$ so that the values of $A$ and $B$ in equation (57) can be written as

$$
\begin{aligned}
& A=(l-2)^{2}-12+4(l-2) \kappa^{2}-(l-3) l^{2} \kappa^{4}+\mathrm{O}\left(\kappa^{6}\right) \\
& B=-l(8-l)-2 l(l-4) \kappa^{2}-\frac{1}{2}\left(6-(l-4)^{2}\right) l^{2} \kappa^{4}+\mathrm{O}\left(\kappa^{6}\right) .
\end{aligned}
$$

As $l$ increases, the real part of the roots for $\sigma$, with $l \approx 2+2 \sqrt{3}$, becomes positive where $A$ changes sign. This shows that the instability appears earlier, at some non-zero wavenumbers, 


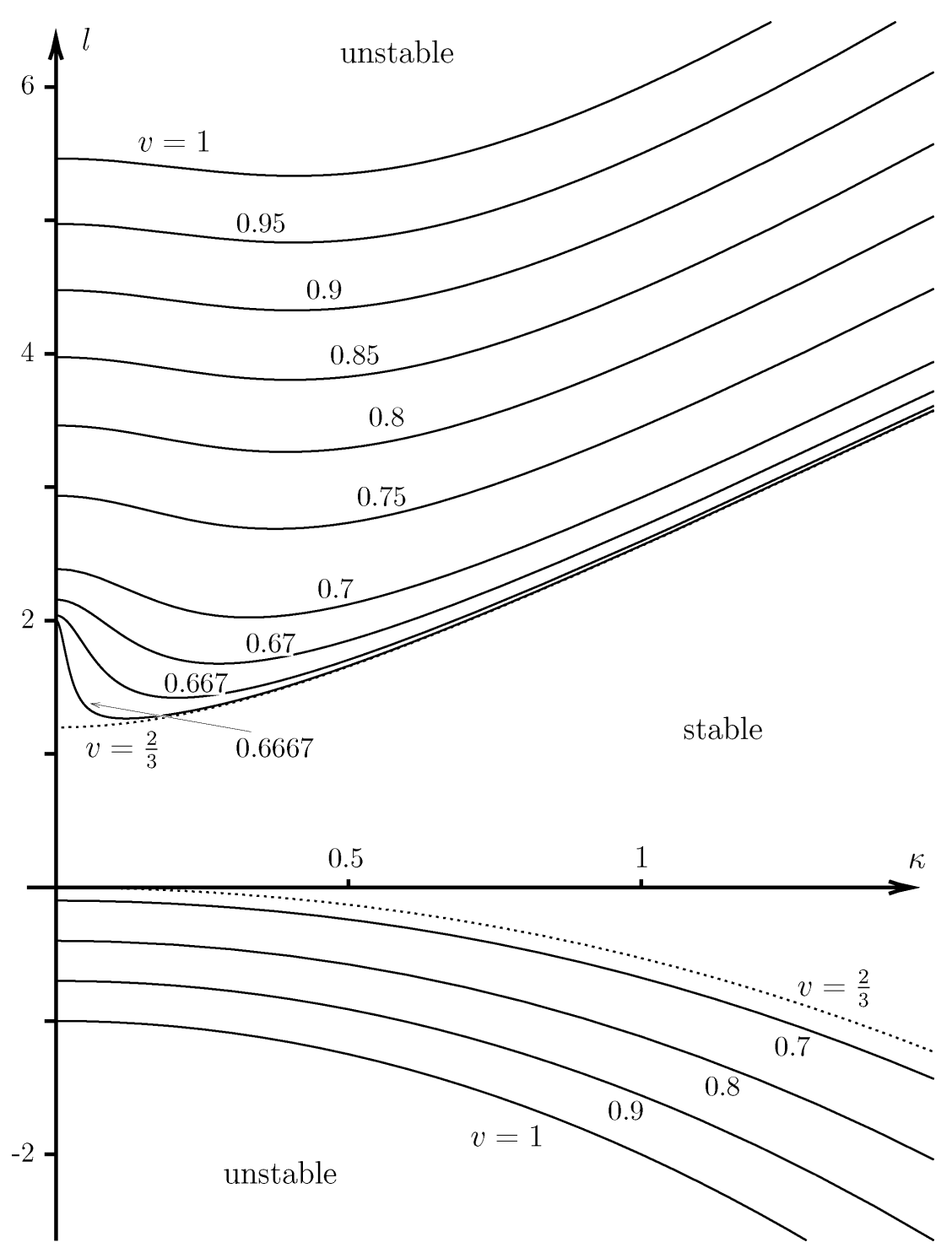

Figure 10. Stability boundaries in the space of the scaled wavenumber $\kappa$ and reduced Lewis number $l$ for fixed values of $v=S / S_{\mathrm{m}}$. The boundaries for $l>0$ correspond to oscillatory modes of instability and those for $l<0$ correspond to cellular modes.

than it does at wavenumber zero. If the terms of order $\kappa^{6}$ are ignored then the instability is predicted to first appear when

$$
l \approx 2+\sqrt{12-2(l-2) \kappa^{2}} \approx 2+2 \sqrt{3}-0.097, \quad \kappa^{2} \approx \frac{2(l-2)}{l^{2}(l-3)} \approx 0.33
$$

although this can only be a rough estimate, without considering the dependence of $r$ on $\kappa$ in greater detail. The value of $B$ is still negative at this stage (in fact more negative) so that the instability is oscillatory in nature, representing travelling or standing-wave disturbances that grow in magnitude.

Solving the dispersion relation (54) numerically for the roots of $\sigma$ leads to the marginalstability boundaries shown in figure 10 . These numerical results both confirm the analytical descriptions already given and extend them over wider ranges of the value of $v$. In reducing $v$ from its adiabatic value of $v=1$, the range of the reduced Lewis number $l$ where stability is encountered is steadily diminished. On approaching the turning point where $v=\frac{2}{3}$, from above, stability is only encountered for $0 \leq l \leq l_{\mathrm{m}} \approx \frac{6}{5}$ (between the dotted paths in the figure). 
This is a narrower range than was predicted for zero wavenumber, since $l_{\mathrm{m}}<2$. Below the turning point all solutions are unstable.

\subsection{Relationship to a one-step model}

The nature of the flame stability identified above (and in figure 10) for flames in which the branching and completion chemistry are all modelled to occur in a thin interface, parallels the stability properties of flames modelled using one-step chemistry at a large activation energy [5]. The difference lies mainly in the definition of terms, such as the reduced Lewis number $l$ of the fuel which, in the present study, depends on $Q-1$ rather than a dimensionless activation energy. The reason for this can be uncovered by examining more closely the relationship with a one-step chemical model for flames.

Flames modelled using one-step chemistry have been studied extensively [9]. Very briefly, for a reaction of the form of (1) the dimensional fuel mass fraction $Y_{\mathrm{F}}$ and temperature $T$ satisfy the equations

$$
\begin{aligned}
\rho\left(Y_{\mathrm{F} t}+\mathbf{u} \cdot \nabla Y_{\mathrm{F}}\right) & =\rho D_{\mathrm{F}} \nabla^{2} Y_{\mathrm{F}}-W_{\mathrm{F}} \omega_{1} \\
\rho C_{\mathrm{p}}\left(T_{t}+\mathbf{u} \cdot \nabla T\right) & =\lambda \nabla^{2} T+Q_{1} \omega_{1}-\ell \\
\omega_{1} & =A_{1} \frac{\rho Y_{\mathrm{F}}}{W_{\mathrm{F}}} \frac{\rho}{W} \mathrm{e}^{-T_{\mathrm{A} 1} / T}
\end{aligned}
$$

with heat of reaction $Q_{1}$ and typically large activation temperature $T_{\mathrm{A} 1}$. Unburnt (or cold) boundary conditions are usually taken to have the form $\lim _{x \rightarrow-\infty}\left(Y_{\mathrm{F}}, T\right)=\left(Y_{\mathrm{Fc}}, T_{\mathrm{c}}\right)$.

If $T_{\mathrm{o}}$ is a reference temperature, which might be close to the adiabatic flame temperature $T_{\mathrm{c}}+Q_{1} Y_{\mathrm{Fc}} /\left(C_{\mathrm{p}} W_{\mathrm{F}}\right)$, then the rescalings

$$
t=t_{\mathrm{o}} t^{\prime}, \quad \mathbf{x}=r_{\mathrm{o}} \mathbf{x}^{\prime}, \quad T=T_{\mathrm{o}} T^{\prime}, \quad Y_{\mathrm{F}}=Y_{\mathrm{Fc}} F
$$

along with the definitions, in which $c$ is a positive constant

$$
\begin{aligned}
& r_{\mathrm{o}}^{2}=\frac{t_{\mathrm{o}} \lambda}{\rho C_{\mathrm{p}}}, \quad \mathbf{u}^{\prime}=\frac{t_{\mathrm{o}}}{r_{\mathrm{o}}} \mathbf{u}, \quad Q^{\prime}=\frac{Q_{1} Y_{\mathrm{Fc}} / W_{\mathrm{F}}}{C_{\mathrm{p}}\left(T_{\mathrm{o}}-T_{\mathrm{c}}\right)}, \\
& \theta_{1}=\frac{T_{\mathrm{A} 1}}{T_{\mathrm{o}}}, \quad t_{\mathrm{o}}=\frac{C_{\mathrm{p}} D_{\mathrm{F}} W}{2 \lambda A_{1}} c^{2} \theta_{1}^{2} \mathrm{e}^{\theta_{1}}, \quad \ell^{\prime}=\frac{\ell t_{\mathrm{o}}}{\rho C_{\mathrm{p}} T_{\mathrm{o}}}
\end{aligned}
$$

lead to the dimensionless equations for $F$ and $T$ (after dropping the primes)

$$
\begin{aligned}
\operatorname{Le}_{\mathrm{F}}\left(F_{t}+\mathbf{u} \cdot \nabla F\right) & =\nabla^{2} F-\frac{1}{2} \theta_{1}^{2} c^{2} F \exp \left(\theta_{1}-\theta_{1} / T\right) \\
T_{t}+\mathbf{u} \cdot \nabla T & =\nabla^{2} T+\frac{1}{2} \theta_{1}^{2} c^{2} \frac{1-T_{\mathrm{c}}}{\mathrm{Le}_{\mathrm{F}}} Q F \exp \left(\theta_{1}-\theta_{1} / T\right)-\ell .
\end{aligned}
$$

The cold boundary conditions become $\lim _{x \rightarrow-\infty}(F, T)=\left(1, T_{\mathrm{c}}\right)$, with $T_{\mathrm{c}}$ now dimensionless.

Assuming that a thin reaction region occurs near $\mathbf{x}=\mathbf{R}$, around a dimensionless temperature $\bar{T}$ that is close to unity in value, $\bar{T}=1+\mathrm{O}\left(\theta_{1}^{-1}\right)$, then the inner asymptotic expansion, for $\theta_{1} \gg 1$

$$
F \sim \theta_{1}^{-1} f(\eta), \quad T \sim \bar{T}-\theta_{1}^{-1} s(\eta), \quad \mathbf{x}-\mathbf{R}=\frac{\widehat{\mathbf{n}} \eta}{c \theta_{1} \exp \left(\theta_{1}(\bar{T}-1) / 2\right)}
$$

leads to the inner model equations

$$
f_{\eta \eta}=\frac{\mathrm{Le}_{\mathrm{F}} / Q}{1-T_{\mathrm{c}}} s_{\eta \eta}=\frac{1}{2} f \exp (-s)
$$


for which burnt matching conditions (where $1 \ll-\eta \ll \theta)$ are $f(-\infty)=s(-\infty)=0$, in which a suitable definition of the flame temperature $\bar{T}$ is implied. It follows that

$$
f=\frac{\mathrm{Le}_{\mathrm{F}} / Q}{1-T_{\mathrm{c}}} s, \quad\left(s_{\eta}\right)^{2}=\int_{0}^{s} \nu \mathrm{e}^{-v} \mathrm{~d} v=1+(1+s) \exp (-s)
$$

from which the values of $F$ and $T$ in the outer region where $\theta^{-1} \ll|n| \ll 1$ can be seen to have the form

$$
\frac{1-T_{\mathrm{c}}}{\mathrm{Le}_{\mathrm{F}}} Q F \sim \bar{T}-T \sim \begin{cases}n c \exp \left(\theta_{1}(\bar{T}-1) / 2\right) & : n>0 \\ 0 & : n<0\end{cases}
$$

in which it is assumed that burnt gas is found where $n<0$. The location of the interface $\mathbf{R}$ is defined such that these straight line asymptotic forms of $F$ and $T$, for $n>0$ and $n<0$, intersect at $n=0$.

These asymptotic solutions can now be expressed in the form of the jump conditions

$$
[T]=[F]=F=0, \quad \frac{1-T_{\mathrm{c}}}{\mathrm{Le}_{\mathrm{F}}} Q\left[F_{n}\right]=-\left[T_{n}\right]=c \exp \left(\theta_{1}(T-1) / 2\right)
$$

to be satisfied by $F$ and $T$, as functions in an outer asymptotic structure with all reaction taken to occur at the interface $\mathbf{x}=\mathbf{R}$. The conditions are the same if the direction of $\widehat{\mathbf{n}}$ is reversed. Away from the interface the equations to be satisfied by $F$ and $T$ are (48), as found when all branching and completion chemistry occurs in a thin interface. The jump conditions (59) also become the same as (47), in the case where $T_{n}^{+}+T_{n}^{-} \neq 0$, if

$$
c \exp \left(\theta_{1}(T-1) / 2\right)=2(T-1)
$$

when evaluated at the interface.

In fact solutions for flame structure and the stability properties of the flames will be the same if the derivatives of both sides of this equation with respect to temperature are also equal at the interface. For this to happen, the value of $c$ and of the 'equivalent' dimensionless one-step activation energy $\theta_{1}$ become

$$
\theta_{1}=\frac{2}{T-1}, \quad c=\frac{2}{\mathrm{e}}(T-1)
$$

with $T$ evaluated at the interface, i.e. $T=\bar{T}$. The corresponding reference temperature $T_{\mathrm{o}}$ must also then be defined such that $T_{\mathrm{o}}=\bar{T}_{*} /\left(1+2 / \theta_{1}\right)$, where $\bar{T}_{*}$ is the dimensional version of the flame temperature $\bar{T}$. When $T-1$ is small at the reaction-sheet, the equivalent one-step activation energy $\theta_{1}$ is large and the constant $c$ (which simply ensures that lengths and times are scaled correspondingly) is small.

This makes it clear that the two forms of model, and their stability results, can be linked when $Q-1$ is small. Since the adiabatic flame temperature in the chain-branching model is $T_{\mathrm{c}}+\left(1-T_{\mathrm{c}}\right) Q$, the equivalent one-step dimensionless activation energy for $Q-1 \ll 1$ becomes $\theta_{1}=2 /\left(\left(1-T_{\mathrm{c}}\right)(Q-1)\right)$ in the case of adiabatic flames. The reduced Lewis number $l$ can then be written as

$$
l=\frac{\mathrm{Le}_{\mathrm{F}}-1}{Q-1}=\frac{1}{2}\left(1-T_{\mathrm{c}}\right) \theta_{1}\left(\mathrm{Le}_{\mathrm{F}}-1\right)
$$

in which $\left(1-T_{\mathrm{c}}\right) \theta_{1}$ can be identified as the equivalent Zel'dovich number. With this means of translating between the two forms of model, the results obtained from the two different approaches become directly analogous to each other, at least in terms of technical details.

The main difference lies in the fact that, in the one-step model, $\theta_{1}$ changes relatively little as the flame temperature, or the reference temperature $T_{\mathrm{o}}$, changes. Thus, for example, the 
reduced Lewis number $l$ would not change nearly as much through changes in $Q$ for the one-step model, as it does for the chain-branching and recombination model when $Q$ is close to unity. Stability predictions in terms of the actual fuel Lewis number $\operatorname{Le}_{\mathrm{F}}$ can therefore be significantly different in detail, even though the broad overall features do parallel each other.

\section{Conclusions}

The chain branching model (2) and its variation (9) offer a useful description for premixed flames, providing more generality than the one-step model (1) as well as a closer connection with the kinds of processes that occur in real hydrogen and hydrocarbon oxidation. The partial degree of chain branching that is allowed for in the version (9) is readily scaled out of the problem, so that all essential features are in fact captured by the simpler model (2). In contrast to the model (3) of Zel'dovich [3,16] analytical asymptotic solutions become possible when density and transport processes are modelled suitably. The main development is that the branching model (2) allows for the effects of an intermediate energetic reactant that acts as a buffer between fuel consumption and heat release.

When the branching reaction is treated as having a large activation energy the fuel is found to be converted into the intermediate reactant at a thin interface, across which relatively simple jump conditions apply. These jump conditions are in fact linear to second order if the temperature at which the branching occurs is taken to be a reference temperature for the nondimensionalization. If a cruder estimate of this branching temperature is used then the conditions are linear to leading order for large activation energy. This leads to straightforward asymptotic descriptions of premixed flames and their stability in a thermodiffusive model with constant coefficients. Solutions are characterized by three properties, namely the temperature and the mass fractions of the fuel and of the intermediate reactant. The general trends of the solutions that are obtained in this way, to second order, are found to be similar to those observed in lean methane and hydrogen flames (see the Appendix).

A further asymptotic reduction of the model arises under conditions in which the maximum flame temperature exceeds a minimum threshold temperature for branching chemistry by a relatively small amount. It becomes sufficient then to use only temperature and the mass fraction of fuel to characterize the solutions in what proves to be a rather simple model formulation; all of the chemistry is found to occur at a thin interface, still involving simple linear jump conditions. The comparisons with lean methane and hydrogen flames in air, that are presented in the Appendix, involve situations in which the flame temperature is not very much in excess of the temperature at which the fastest branching chemistry occurs (producing hydrogen atoms $\mathrm{H}^{\cdot}$ ). The simpler model that arises in this situation may therefore be fairly widely applicable, certainly near any lean flammability limit.

The jump conditions for this simple model resemble the nonlinear (and so more complicated) jump conditions that arise from one-step chemistry sufficiently closely for an 'equivalent' onestep activation energy to be identified. A one-step model with this activation energy would lead to completely analogous solutions to leading order. However, the equivalent activation energy depends strongly on the solution and it grows towards infinity as the flame temperature decreases towards its threshold value for branching. The assumptions underlying the two different approaches are therefore not compatible (most particularly that the one-step activation temperature is a large constant) and care is needed in interpreting results, such as flame-speed and stability boundaries, from one approach in the context of the other. Also, the close leading order connection between the models that arise from the different assumptions concerning the chemistry cannot be expected to extend to higher orders [32]. 
Stability analyses of flames with branching chemistry, described using either of the two levels of asymptotic reduction that have been found, predict the two forms of instability that are known for one-step chemistry [5], as well as the complete loss of stability on the lower branch of flame speeds for heat losses below a critical heat loss for extinction. The fuller model, which includes the mass fraction of the intermediate reactant in the description of flames, allows for a deeper insight into the ranges of conditions in which instability can appear.

The oscillatory instability that is predicted for a large enough fuel Lewis number is found to disappear altogether in adiabatic flames as the heat of reaction increases above a moderately low level. Even at low enough heats of reaction the Lewis number required for this instability is probably too high for it to be found in any realistic type of adiabatic gaseous premixed flame. However, heat losses have the effect of reducing the marginal Lewis number which increases the likelihood of developing the oscillatory instability. The cellular instability is predicted to arise at low enough Lewis numbers, below unity in the adiabatic case. The marginal fuel Lewis number for this increases towards unity as the heat of reaction is decreased. It also increases with increased heat losses and can exceed unity in cases that are close to extinction provided the Lewis number of the intermediate species is also greater than unity.

The predicted marginal Lewis numbers for instability, as well as the underlying propagating flame solutions themselves, depend on the heat of reaction and on the degree of heat loss but not on the activation energy of the branching reaction, to leading order. Higher order effects introduce a relatively weak dependence on the branching activation energy that is consistent with the trends that can be expected of real hydrogen and hydrocarbon flames. By contrast the solutions describing flames that arise from a one-step model for the chemistry depend intimately (in fact exponentially) on the activation energy of the reaction step; although the marginal Lewis numbers for instability of these flames vary relatively weakly with the heat of reaction and activation energy, through the Zel'dovich number, the time and length scales of the instabilities are strongly dependent on these quantities.

The relative independence of the solutions and their stability on the value of the branching activation energy is probably the most significant structural and dynamical consequence of the buffering role of the intermediate reactant. All that is needed is for the branching chemistry to be thermally sensitive enough to ensure that the branching reaction occurs in a thinner layer than the completion reaction. The rate of branching chemistry (and hence the rate of production of the intermediate) is then diffusion-limited by the rate at which fuel can enter the reaction layer. There is no immediate connection between the thickness of the layer where fuel is consumed and the size of the region where heat is generated, as the intermediate reactant diffuses and is consumed over a range that depends little on the thickness of the layer in which it is produced. Solutions then depend primarily on the heat of reaction and the degree of heat loss, as well as the Lewis numbers of fuel and of the intermediate reactant.

The actual temperature of the layer in which branching chemistry occurs does depend on the activation energy of the branching reaction, although this is a second-order effect for large activation energy. However, since dimensionless values of the activation energy are not generally very large (roughly five in value) these higher order effects are fairly significant. It has been shown in equations (28) and (30), or (49), that the temperature of the branching reaction layer depends on the solution. This contributes to the trends that are found through varying conditions, such as the equivalence ratio, as outlined in the Appendix.

The chain-branching chemical model that is presented and examined here does not take into account the role of an oxidant. Work is proceeding in examining variations of the model that do account for both fuel and oxidant so that changes in stoichiometry can be admitted and both rich and lean premixed flames can be described, as well as diffusion flames. The inclusion of a non-zero heat of reaction for the branching step is another development that is in progress. 


\section{Acknowledgements}

Conversations with a number of people have helped in the development of the ideas presented in this article. In particular the author would like to thank Charlie Westbrook, Forman Williams, Barry Greenberg, Grisha Sivashinsky, Kal Seshadri, Amable Liñán, Rodney Weber, Joel Daou and Norbert Peters for useful discussions and suggestions (that were not always followed). Funding from the EPSRC is gratefully acknowledged as is the much valued assistance of Anna Zinoviev.

\section{Appendix: Lean premixed flames}

The solutions (36) and (37) are based on taking the limit $\theta \rightarrow \infty$ whereas more realistic values of $\theta$ seem to be about five. Nevertheless, a value of $\theta \approx 5$ can still be considered to be large enough for higher-order asymptotic estimates to have some significance. Not only does this allow relationships between $T_{\mathrm{r}}$ and $T_{\times}$to be calculated, through (19), it also allows the dimensional value of the temperature at the branching reaction sheet to be calculated from (28) or (30), or (49) where appropriate. Since the leading-order dimensionless flamesheet calculations based on (32) all take this temperature to be exactly unity, this temperature is a very suitable one to choose as the scaling temperature $T_{\mathrm{s}}$, rather than the more crudely estimated reference temperature $T_{\mathrm{r}}$.

Defining $T_{\mathrm{s}}$ in this way, equations (19), (28) and (30) can be used to estimate $T_{\mathrm{s}}$ as

$$
T_{\mathrm{s}} \approx \begin{cases}T_{\times}+T_{\times} \frac{T_{\mathrm{s}}}{T_{\mathrm{B}}}\left(\ln \frac{T_{n}^{\prime 2}}{Z}-2 \gamma+2 \ln \frac{T_{\mathrm{B}}}{T_{\mathrm{s}}}\right) & : T_{n} \neq 0 \\ T_{\times}+T_{\times} \frac{T_{\mathrm{S}}}{T_{\mathrm{B}}}\left(\ln \frac{\left[F_{n}\right]}{F Z}+\frac{1}{2} \ln \frac{\left(1-T_{\mathrm{c}} / T_{\mathrm{S}}\right) Q^{\prime} Z / \mathrm{Le}_{\mathrm{F}}-\ell^{\prime}}{2 \pi}+\frac{1}{2} \ln \frac{T_{\mathrm{B}}}{T_{\mathrm{s}}}\right) & : T_{n}=0\end{cases}
$$

in which $\theta$ has been taken to represent $T_{\mathrm{B}} / T_{\mathrm{s}}$, which is probably the most suitable definition of the dimensionless activation temperature for a branching reaction-sheet at the temperature $T_{\mathrm{s}}$. Errors that arise from the use of this second-order asymptotic formula for $T_{\mathrm{B}} \gg T_{\mathrm{S}}$ can be estimated to be of the order of $T_{\mathrm{s}}^{3} / T_{\mathrm{B}}^{2}$, or very roughly $30 \mathrm{~K}$ to $100 \mathrm{~K}$ for the values shown later in figure 11.

The terms $T_{n}^{\prime}, Z,\left[F_{n}\right], F$ and $\ell^{\prime}$ are all dimensionless and evaluated at the interface. Primes emphasize the dimensionless nature of a term. It follows that the value of $T_{\mathrm{s}}$ and hence some of the dimensionless terms defined in (21) and (22) are then weakly dependent on the solutions through the higher-order contributions appearing in (60). On the other hand, the solutions based on the branching-sheet model (31) and jump conditions (32) are then accurate to second-order for $\theta \gg 1$. With the reference temperature $T_{\mathrm{s}}$ defined in this way, some care must therefore be exercised in comparing the asymptotic flame solutions with more realistic flames, even ignoring the fact that density and transport properties have been taken to be constant in the simple model formulation.

Nevertheless, some broad features are relatively easily identified for comparison with lean flames calculated using fuller chemical kinetic models, having an upstream (cold boundary) dimensional temperature of $T_{\mathrm{c}}$ and mass fraction of $Y_{\mathrm{Fc}} \approx \phi Y_{\mathrm{F} 1}$, for an 'equivalence ratio' of $\phi<1$. With a finite branching activation temperature $T_{\mathrm{B}}$ the dimensional crossover temperature $T_{\times}$then depends on $\phi$ through equation (15) in the manner

$$
\frac{T_{\mathrm{B}}}{T_{\times}} \approx \ln \phi+\frac{T_{\mathrm{B}}}{T_{\times 1}}
$$

where $T_{\times 1}$ is the crossover temperature extrapolated to $\phi=1$. 

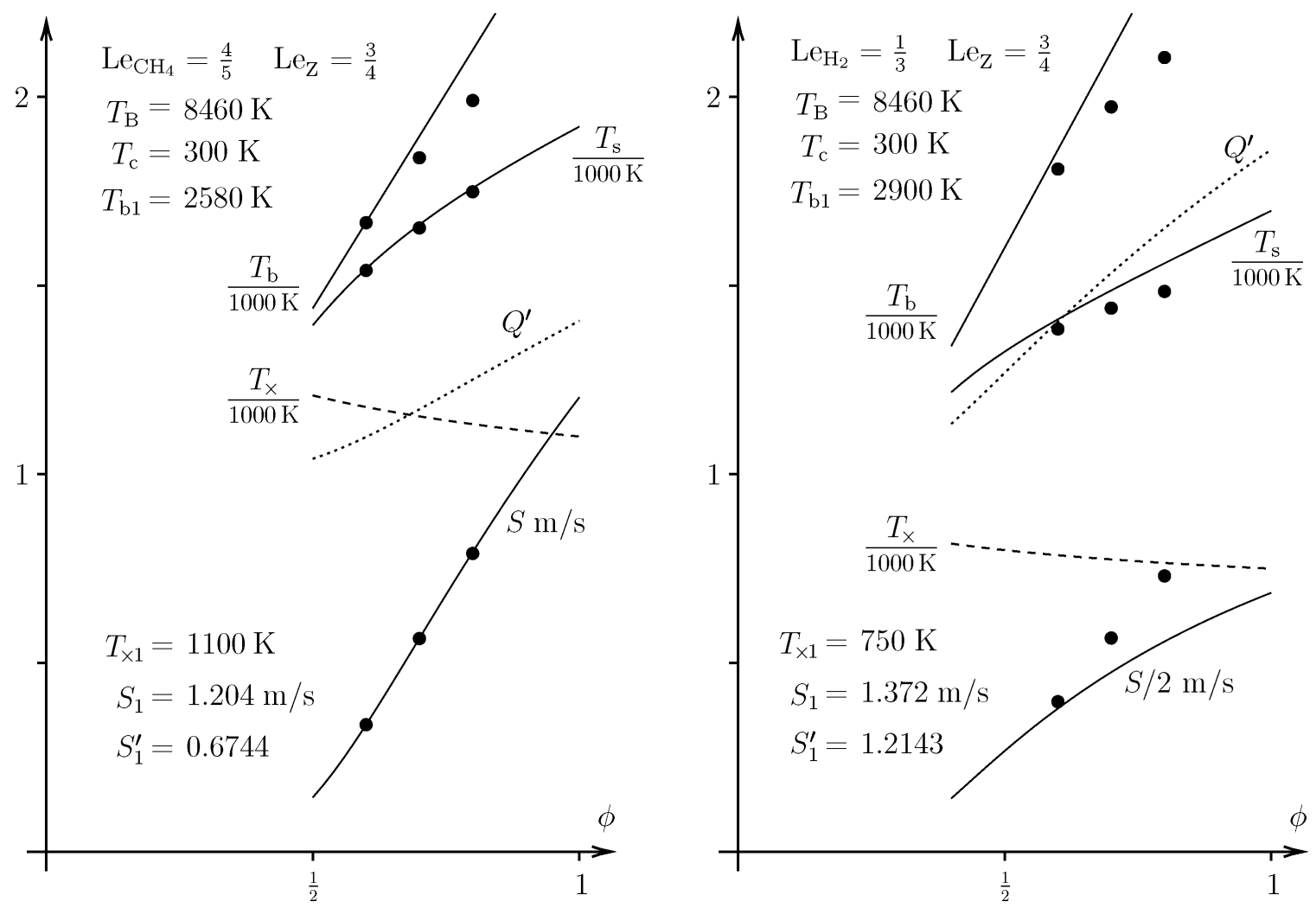

Figure 11. Values of the crossover temperature $T_{\times}$(shown as a dashed curve), branching-sheet temperature $T_{\mathrm{s}}$, adiabatic burnt temperature $T_{\mathrm{b}}$, flame speed $S$ and dimensionless heat of reaction $Q^{\prime}$ (dotted curve) that appear to be broadly representative of lean methane-air flames (left) and lean hydrogen-air flames (right) in a mixture with equivalence ratio $\phi$ at an unburnt temperature of $T_{\mathrm{c}}=300 \mathrm{~K}$. The curves of temperatures $T_{\times}, T_{\mathrm{s}}$ and $T_{\mathrm{b}}$ are all scaled by dividing by $1000 \mathrm{~K}$ while the flame-speed $S$ is divided by 2 in the right-hand graph. The dark circles show values of $T_{\mathrm{b}}, T_{\mathrm{s}}$ and $S$ that are calculated from a full chemistry and transport model using COSILAB [33], with the branching-sheet temperature $T_{\mathrm{s}}$ estimated as the temperature at the point where the concentration of $\mathrm{H}^{\cdot}$ atoms reaches its maximum value.

The adiabatic case is the simplest to consider, although nonadiabatic cases would be examined in the same general way using suitably modified formulae. The solutions (36) and (37) then give

$$
\frac{T_{n}^{\prime 2}}{Z}=\frac{Q^{\prime 2} S^{\prime}}{\left(1-S^{\prime 2}+\mathrm{Le}_{\mathrm{Z}} S^{\prime 2}\right)^{2}} \frac{\sqrt{4+\mathrm{Le}_{\mathrm{Z}}^{2} S^{\prime 2}}}{\mathrm{Le}_{\mathrm{F}}}
$$

in which

$$
2 Q^{\prime}\left(\sqrt{4+\mathrm{Le}_{\mathrm{Z}}^{2} S^{\prime 2}}+S^{\prime}\right)=\left(2+S^{\prime} \sqrt{4+\mathrm{Le}_{\mathrm{Z}}^{2} S^{\prime 2}}+\mathrm{Le}_{\mathrm{Z}} S^{\prime 2}\right) \sqrt{4+\mathrm{Le}_{\mathrm{Z}}^{2} S^{\prime 2}}
$$

It is also useful to identify the adiabatic burnt temperature $T_{\mathrm{b}}$ and dimensionless heat-release $Q^{\prime}$ as satisfying

$$
T_{\mathrm{b}}-T_{\mathrm{c}} \approx\left(T_{\mathrm{s}}-T_{\mathrm{c}}\right) Q^{\prime} \approx \phi\left(T_{\mathrm{b} 1}-T_{\mathrm{c}}\right)
$$

from (13) and (22), where $T_{\mathrm{b} 1}$ is the adiabatic burnt temperature extrapolated to $\phi=1$.

The scalings (21) and (22) show that the dimensional flame speed $S$ is given by

$$
S=S^{\prime} \frac{\lambda}{\rho C_{\mathrm{p}}}\left(\frac{\rho A_{\mathrm{C}}}{D_{\mathrm{Z}} W}\right)^{1 / 2} \approx S^{\prime} \times \frac{S_{1}}{S_{1}^{\prime}} .
$$


Thus the scaling between $S$ and $S^{\prime}$ does not depend on the value of the branching sheet temperature $T_{\mathrm{s}}$, so that the ratio $S / S^{\prime}$ at any equivalence ratio $\phi$ should be the same as the ratio $S_{1} / S_{1}^{\prime}$, extrapolated to $\phi=1$.

It is interesting to note that, for finite values of $\theta$, the value of $T_{\mathrm{s}}$ can be seen to depend weakly on $\mathrm{Le}_{\mathrm{F}}$ through (60) and (62). Because this affects the values of $Q^{\prime}$ and hence $S^{\prime}$, the flame speed that is finally calculated actually does depend weakly on the Lewis number of the fuel, unlike the leading order flame speed calculated as $\theta \rightarrow \infty$.

For fixed values of $\mathrm{Le}_{\mathrm{Z}}, \mathrm{Le}_{\mathrm{F}}, T_{\mathrm{B}}, T_{\mathrm{b} 1}, T_{\times 1}$ and $T_{\mathrm{c}}$ the values of $T_{\mathrm{b}}$ and $T_{\times}$can be calculated readily from (61) and (64) for any suitable value of $\phi<1$. However, the values of $T_{\mathrm{s}}, Q^{\prime}$ and $S^{\prime}$ can only be found by solving the coupled equations (60) to (64). This is a straightforward task if carried out numerically.

Figure 11 shows how the flame speed $S$, the dimensionless heat of reaction $Q^{\prime}$ and the temperatures $T_{\times}, T_{\mathrm{S}}$ and $T_{\mathrm{b}}$ vary with $\phi$ in cases that seem to be broadly representative of lean hydrogen-air and lean methane-air flames. The dark circles shown in the figure are based on detailed calculations of these flames, with the branching-sheet temperature $T_{\mathrm{s}}$ estimated to be the temperature at which the concentration of hydrogen atoms $\mathrm{H}$ reaches its maximum value. The calculations are not particularly sensitive to the choice of $\mathrm{Le}_{\mathrm{Z}}$, so that the value $\mathrm{Le}_{\mathrm{Z}} \approx \frac{3}{4}$ is not necessarily the best choice although it does seem reasonable. The curves are more sensitive to the choices of $T_{\mathrm{b} 1}, T_{\times 1}$ and $S_{1}$. The branching activation temperature $T_{\mathrm{B}}$ is assigned the value $T_{\mathrm{B}}=8460 \mathrm{~K}$, namely the activation temperature of the reaction $\mathrm{H}^{\cdot}+\mathrm{O}_{2} \rightarrow \mathrm{OH}^{\cdot}+\mathrm{O}$.

It can be seen that the calculations manage to approximate methane flames quite well with crossover temperatures $T_{\times}$between about $1100 \mathrm{~K}$ and $1200 \mathrm{~K}$ and branching reaction-sheet temperatures $T_{\mathrm{s}}$ between about $1400 \mathrm{~K}$ and $1900 \mathrm{~K}$ over the range of values of $\phi$ that is shown. The approximation of the hydrogen flame improves as $\phi$ decreases, with suitable values of $T_{\times}$ seeming to fall between about $750 \mathrm{~K}$ and $900 \mathrm{~K}$ and with $T_{\mathrm{s}}$ between about $1200 \mathrm{~K}$ and $1600 \mathrm{~K}$. The values of the dimensionless heat of reaction $Q^{\prime}$ are not very large for the methane-air flame, approaching about 1.4 at $\phi=1$. For the hydrogen-air flame values of $Q^{\prime}$ approach about 1.8. At lower equivalence ratios, the values of $Q^{\prime}$ become close to unity, which shows that the limit $Q^{\prime}-1 \ll 1$ can be a reasonable approximation, especially when approaching the flammability limit. The trend is for the values of $T_{\times}, T_{\mathrm{S}}$ and $T_{\mathrm{b}}$ to converge towards each other as $\phi$ is decreased.

Some caution must be exercised in interpreting these comparisons with hydrogen and hydrocarbon flames. Firstly, the simple model used here involves constant density and transport coefficients so that the comparisons in figure 11 should be seen as no more than broadly indicative. Secondly, the second-order asymptotics applied for $\theta \approx 5$, or thereabouts, must involve some uncertainty, particularly in estimating the relationship between $T_{\times}$and $T_{\mathrm{s}}$. Thirdly, the simple branching model involves fuel and no oxidant whereas hydrogen and hydrocarbon flames must involve both. Over the range of equivalence ratio shown in figure 11 some change in oxidant concentration must occur which should be expected to have an influence.

In spite of this, the results appear to demonstrate the correct trends at least. Crossover temperatures $T_{\times}$for hydrogen are significantly lower than those for methane (and probably other hydrocarbons) although their values seen in figure 11 for both of these cases may be estimated as being too low; crossover temperatures for homogeneous chain branching are normally found to have moderately higher values $[9,17]$. Thermal expansion and temperature dependence of the transport coefficients may partly account for the discrepancy, as well as limitations in the accuracy of the asymptotic approximations.

On the other hand, it is worth stressing that the actual chemical chain-branching process in these flames takes place as a reactive-diffusive balance around the temperature $T_{\mathrm{s}}$. The crossover temperature $T_{\times}$has no direct physical relevance in the flame structure and, 
in this context, it is merely an extrapolation to a notional homogeneous-chemistry reference point from the highly reactive mixture conditions pertaining in the flame where the actual chain branching predominates. The conditions experienced during genuinely homogeneous self-ignition at a chemical crossover temperature in hydrogen or hydrocarbon oxidation might be significantly different in a way that the model is not able to capture fully. Of more direct physical relevance to propagating premixed flames are the values estimated for the branching reaction-sheet temperature $T_{\mathrm{s}}$. These do seem to follow the correct trend, as do the calculated flame speeds $S$, especially in the case of the methane-air flames.

\section{References}

[1] Williams, F.A., 1971, Theory of combustion in laminar flows. Annual Reviews of Fluid Mechanics, 3, 171188.

[2] Williams, F.A., 1965, Combustion Theory (New York: Addison-Wesley); Second edition: 1985 (California: Benjamin-Cummings).

[3] Zel'dovich, Ya.B., Barrenblatt, G.I., Librovich, V.B., and Makhviladze, G.M., 1985, The Mathematical Theory of Combustion and Explosions (New York: Consultants Bureau).

[4] Buckmaster, J.D. and Ludford, G.S.S., 1982, Theory of Laminar Flames (Cambridge: Cambridge University Press).

[5] Sivashinsky, G.I., 1983, Instabilities, pattern formation and turbulence in flames. Annual Reviews of Fluid Mechanics, 15, 179-199.

[6] Kapila, A.K., 1983, Asymptotic Treatment of Chemically Reacting Systems (London: Pitman).

[7] Pelcé, P., 1988, Dynamics of Curved Fronts (London: Academic Press).

[8] Buckmaster, J., 1993, The structure and stability of laminar flames. Annual Reviews of Fluid Mechanics, 25, 21-53.

[9] Liñán, A. and Williams, F.A., 1993, Fundamental Aspects of Combustion (New York: Oxford University Press).

[10] Peters, N., 2000, Turbulent Combustion (Cambridge: Cambridge University Press).

[11] Buckmaster, J., Clavin, P., Liñán, A., Matalon, M., Peters, N., Sivashinsky, G., and Williams F.A., 2005, Combustion theory and modeling. Proceedings of the Combustion Institute, 30, 1-19.

[12] Smooke, M.D. (Ed.), 1991, Reduced Kinetic Mechanisms and Asymptotic Approximations for Methane-Air Flames. Lecture Notes in Physics, vol. 384 (Berlin: Springer-Verlag).

[13] Peters, N. and Rogg, B. (Eds.), 1993, Reduced Reaction Mechanisms for Applications in Combustion Systems. Lecture Notes in Physics, New Series, vol. m15 (Berlin: Springer-Verlag).

[14] Seshadri, K., Peters, N., and Williams F.A., 1994, Asymptotic analyses of stoichiometric and lean hydrogen-air flames. Combustion and Flame, 96, 407-427.

[15] Peters, N., 1997, Kinetic foundation of thermal flame theory. Progress in Astronautics and Aeronautics, 173, 73-91.

[16] Zel'dovich, Ya.B., 1948, K Teorii Raspostraneniya Plameni. Zhurnal Fizicheskoi Khimii, 22, $27-48$.

[17] Westbrook, C.K., 2000, Chemical kinetics of hydrocarbon ignition in practical combustion systems. Proceedings of the Combustion Institute, 28, 1563-1577.

[18] NIST Chemical Kinetics Database (http://kinetics.nist.gov/kinetics).

[19] NIST Standard Reference Database No. 69, June 2005 (http://webbook.nist.gov/chemistry).

[20] Westbrook, C.K. and Dryer, F.L., 1984, Chemical kinetic modeling of hydrocarbon combustion. Progress in Energy and Combustion Science, 10, 1-57.

[21] Kapila, A.K., 1978, Homogeneous branched-chain explosion-Initiation to completion. Journal of Engineering Mathematics, 12, 221-235.

[22] Joulin, G., Liñán, A., Ludford, G.S.S., Peters, N., and Schmidt-Lainé, C., 1985, Flames with chain-branching chain-breaking kinetics. SIAM Journal on Applied Mathematics, 45, 420-434.

[23] Chao, B.H. and Law, C.K., 1994, Laminar flame propagation with volumetric heat-loss and chain branchingtermination reactions. International Journal of Heat and Mass Transfer, 37, 673-680.

[24] Mikolaitis, D.W., 1986, Adiabatic flame speeds and the Zel'dovich-Liñán model. Combustion Science and Technology, 49, 277-288.

[25] Tam, R.Y., 1988, Stretch response and large heat release in the Zel'dovich-Liñán model. Combustion Science and Technology, 60, 125-142.

[26] Tam, R.Y., 1988, Damköhler number ratio asymptotics of the Zel'dovich-Liñán model. Combustion Science and Technology, 62, 297-309.

[27] Seshadri, K. and Peters, N., 1983, The influence of stretch on a premixed flame with 2-step kinetics. Combustion Science and Technology, 33, 35-63.

[28] Dold, J.W., Thatcher, R.W., Omon-Arancibia, A., Redman, J., 2002, From one-step to chain-branching premixed-flame asymptotics. Proceedings of the Combustion Institute, 29 1519-1526. 
[29] Dold, J.W., Daou, J., and Weber, R., 2004, Reactive-diffusive stability of premixed flames with modified Zel'dovich-Liñán kinetics. In: F.J. Higuera, J. Jimenez and J.M. Vega (Eds.) Simplicity, Rigor and Relevance in Fluid Mechanics (Barcelona: CIMNE publishers).

[30] Dold, J.W., Weber, R.O., Thatcher, R.W., and Shah, A.A., 2003, Flame balls with thermally sensitive intermediate kinetics. Combustion Theory and Modelling, 7, 175-203.

[31] Abramowitz, M. and Stegun, I.A., 1972, Handbook of Mathematical Functions (New York: Dover Publications).

[32] Dold, J.W., Thatcher, R.W., and Shah, A.A., 2003, High order effects in one-step reaction-sheet jump conditions for premixed flames. Combustion Theory and Modelling, 7, 109-127.

[33] COSILAB: http://www.softpredict.com 